\title{
Decarboxylative Etherification of Aromatic Carboxylic Acids
}

\author{
Sukalyan Bhadra, $\uparrow$ Wojciech I. Dzik, $\uparrow$ Lukas J. Goossen $† *$ \\ † Department of Organic Chemistry, Technische Universität Kaiserslautern, Erwin- \\ Schrödinger-Str. Geb. 54, 67663 Kaiserslautern, Germany
}

goossen@chemie.uni-kl.de

Supporting Information

Table of contents

Catalyst Optimization S2

Preparative procedures S3-S8 S9-S82 
Table S1. Optimization of the Reaction Conditions for the Decarboxylative Etherification

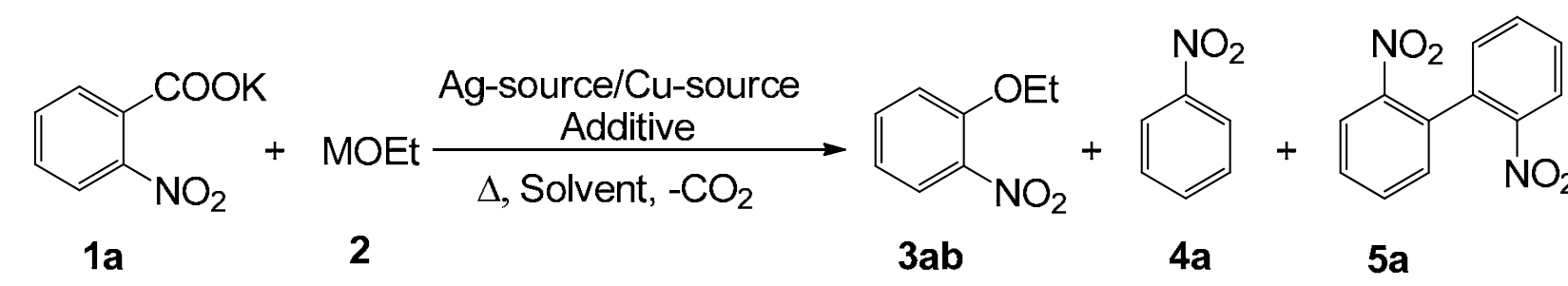

\begin{tabular}{|c|c|c|c|c|c|c|c|c|c|}
\hline \multirow[t]{2}{*}{ entry } & \multirow[t]{2}{*}{ MOEt } & \multirow[t]{2}{*}{ Ag-source } & \multirow[t]{2}{*}{ Cu-source } & \multirow[t]{2}{*}{ additive } & \multirow[t]{2}{*}{ solvent } & \multirow{2}{*}{$\begin{array}{l}\text { temp } \\
\left({ }^{\circ} \mathrm{C}\right)\end{array}$} & \multicolumn{3}{|c|}{ yield (\%) } \\
\hline & & & & & & & 3ab & $4 a$ & $5 a$ \\
\hline $1^{b, c}$ & EtOH & $\mathrm{Ag}_{2} \mathrm{CO}_{3}$ & $\mathrm{Cu}(\mathrm{OAc})_{2}$ & - & $\overline{\mathrm{DMF}}$ & 120 & 0 & 62 & 0 \\
\hline $2^{b}$ & $\mathrm{NaOEt}$ & $\mathrm{Ag}_{2} \mathrm{CO}_{3}$ & $\mathrm{Cu}(\mathrm{OAc})_{2}$ & - & DMF & 120 & 0 & trace & 0 \\
\hline $3^{b}$ & $\mathrm{~B}(\mathrm{OEt})_{3}$ & $\mathrm{Ag}_{2} \mathrm{CO}_{3}$ & $\mathrm{Cu}(\mathrm{OAc})_{2}$ & - & DMF & 120 & 52 & trace & 0 \\
\hline $4^{b}$ & $\mathrm{Si}(\mathrm{OEt})_{4}$ & $\mathrm{Ag}_{2} \mathrm{CO}_{3}$ & $\mathrm{Cu}(\mathrm{OAc})_{2}$ & - & DMF & 120 & 72 & 13 & 5 \\
\hline $5^{b, d}$ & $\mathrm{Si}(\mathrm{OEt})_{4}$ & $\mathrm{Ag}_{2} \mathrm{CO}_{3}$ & $\mathrm{Cu}(\mathrm{OAc})_{2}$ & o-phen & DMF & 120 & 35 & 14 & 8 \\
\hline $6^{b, d}$ & $\mathrm{Si}(\mathrm{OEt})_{4}$ & $\mathrm{Ag}_{2} \mathrm{CO}_{3}$ & $\mathrm{Cu}(\mathrm{OAc})_{2}$ & lutidine & DMF & 120 & 45 & 30 & 0 \\
\hline 7 & $\mathrm{Si}(\mathrm{OEt})_{4}$ & $\mathrm{Ag}_{2} \mathrm{CO}_{3}$ & $\mathrm{Cu}(\mathrm{OAc})_{2}$ & BQ & DMF & 120 & 0 & 62 & 0 \\
\hline 8 & $\mathrm{Si}(\mathrm{OEt})_{4}$ & $\mathrm{Ag}_{2} \mathrm{CO}_{3}$ & $\mathrm{Cu}(\mathrm{OAc})_{2}$ & $\mathrm{~K}_{2} \mathrm{~S}_{2} \mathrm{O}_{8}$ & DMF & 120 & 0 & trace & 0 \\
\hline 9 & $\mathrm{Si}(\mathrm{OEt})_{4}$ & $\mathrm{Ag}_{2} \mathrm{CO}_{3}$ & $\mathrm{Cu}(\mathrm{OAc})_{2}$ & $\mathrm{O}_{2}$ & DMF & 120 & 68 & 15 & 4 \\
\hline 10 & $\mathrm{Si}(\mathrm{OEt})_{4}$ & $\mathrm{Ag}_{2} \mathrm{CO}_{3}$ & $\mathrm{Cu}(\mathrm{OAc})_{2}$ & $\mathrm{O}_{2}$ & DMF & 145 & 81 & trace & 0 \\
\hline 11 & $\mathrm{Si}(\mathrm{OEt})_{4}$ & $\mathrm{Ag}_{2} \mathrm{CO}_{3}$ & $\mathrm{Cu}(\mathrm{OAc})_{2}$ & $\mathrm{O}_{2}$ & DMSO & 145 & 36 & 18 & 0 \\
\hline 12 & $\mathrm{Si}(\mathrm{OEt})_{4}$ & $\mathrm{Ag}_{2} \mathrm{CO}_{3}$ & $\mathrm{Cu}(\mathrm{OAc})_{2}$ & $\mathrm{O}_{2}$ & DMPU & 145 & 41 & 20 & 3 \\
\hline 13 & $\mathrm{Si}(\mathrm{OEt})_{4}$ & $\mathrm{Ag}_{2} \mathrm{CO}_{3}$ & $\mathrm{Cu}(\mathrm{OAc})_{2}$ & $\mathrm{O}_{2}$ & NMP & 145 & 0 & 33 & 0 \\
\hline 14 & $\mathrm{Si}(\mathrm{OEt})_{4}$ & $\mathrm{Ag}_{2} \mathrm{CO}_{3}$ & $\mathrm{Cu}(\mathrm{OAc})_{2}$ & $\mathrm{O}_{2}$ & diglyme & 145 & 29 & 15 & 6 \\
\hline 15 & $\mathrm{Si}(\mathrm{OEt})_{4}$ & $\mathrm{Ag}_{2} \mathrm{CO}_{3}$ & $\mathrm{Cu}(\mathrm{OAc})_{2}$ & $\mathrm{O}_{2}$ & mesitylene & 145 & 0 & 30 & 0 \\
\hline 16 & $\mathrm{Si}(\mathrm{OEt})_{4}$ & - & $\mathrm{Cu}(\mathrm{OAc})_{2}$ & $\mathrm{O}_{2}$ & DMF & 145 & 24 & 23 & 14 \\
\hline 17 & $\mathrm{Si}(\mathrm{OEt})_{4}$ & $\mathrm{Ag}_{2} \mathrm{CO}_{3}$ & - & $\mathrm{O}_{2}$ & DMF & 145 & 0 & trace & 0 \\
\hline 18 & $\mathrm{Si}(\mathrm{OEt})_{4}$ & $\mathrm{AgOAc}$ & $\mathrm{Cu}(\mathrm{OAc})_{2}$ & $\mathrm{O}_{2}$ & DMF & 145 & 18 & 24 & 12 \\
\hline 19 & $\mathrm{Si}(\mathrm{OEt})_{4}$ & $\mathrm{Ag}_{2} \mathrm{O}$ & $\mathrm{Cu}(\mathrm{OAc})_{2}$ & $\mathrm{O}_{2}$ & DMF & 145 & 24 & 19 & 11 \\
\hline 20 & $\mathrm{Si}(\mathrm{OEt})_{4}$ & AgOTf & $\mathrm{Cu}(\mathrm{OAc})_{2}$ & $\mathrm{O}_{2}$ & DMF & 145 & 0 & 22 & 15 \\
\hline 21 & $\mathrm{Si}(\mathrm{OEt})_{4}$ & $\mathrm{Ag}_{2} \mathrm{CO}_{3}$ & $\mathrm{CuBr}_{2}$ & $\mathrm{O}_{2}$ & DMF & 145 & 33 & 0 & 30 \\
\hline 22 & $\mathrm{Si}(\mathrm{OEt})_{4}$ & $\mathrm{Ag}_{2} \mathrm{CO}_{3}$ & $\mathrm{CuCl}_{2}$ & $\mathrm{O}_{2}$ & DMF & 145 & 0 & 0 & 26 \\
\hline 23 & $\mathrm{Si}(\mathrm{OEt})_{4}$ & $\mathrm{Ag}_{2} \mathrm{CO}_{3}$ & $\mathrm{CuF}_{2}$ & $\mathrm{O}_{2}$ & DMF & 145 & 0 & 0 & 0 \\
\hline 24 & $\mathrm{Si}(\mathrm{OEt})_{4}$ & $\mathrm{Ag}_{2} \mathrm{CO}_{3}$ & $\mathrm{Cu}(\mathrm{OTf})_{2}$ & $\mathrm{O}_{2}$ & DMF & 145 & 0 & 0 & 35 \\
\hline
\end{tabular}

${ }^{a}$ Reaction conditions: 0.3 mmol potassium 2-nitrobenzoate, 1.2 equiv. MOEt, 25 mol\% Ag-source, 1 equiv. Cu-source, $2 \mathrm{~mL}$ solvent, $18 \mathrm{~h}$. Yields determined by GC using $n$-tetradecane as internal standard. ${ }^{\mathrm{b}} 1$ equiv. $\mathrm{Ag}_{2} \mathrm{CO}_{3} .{ }^{\mathrm{c}} 20$ equiv. EtOH. ${ }^{\mathrm{d}} 20 \mathrm{~mol} \%$ of $\mathrm{Cu}$-source and $30 \mathrm{~mol} \%$ of additive. 
General Methods. All catalytic reactions were performed in $20 \mathrm{~mL}$ oven-dried crimp-cap vials equipped with a Teflon-coated stirring bar and a septum cap. Solvents were purified by standard procedures prior to use. All reactions were monitored by GC using $n$-tetradecane as an internal standard. Response factors of the products with regard to $n$-tetradecane were obtained experimentally by analyzing known quantities of the substances. Column chromatography was performed using a Combi Flash Companion-Chromatography-System (Isco-Systems) and RediSep packed columns (24 g). NMR spectra were obtained on Bruker AMX 400 or on Bruker Avance 600 systems using $\mathrm{CDCl}_{3}$, methanol- $\mathrm{d}_{4}$ and $\mathrm{D}_{2} \mathrm{O}$ as solvents, with proton and carbon resonances at $400 / 600 \mathrm{MHz}$ and 101/151 MHz, respectively. Mass spectral data were acquired on a GC-MS Saturn $2100 \mathrm{~T}$ (Varian). Melting points were determined with a Mettler FP61 melting point apparatus. Copper salts and silver salts were dried in vacuo at $100{ }^{\circ} \mathrm{C}$, prior to use. All potassium salts were dried for $2 \mathrm{~h}$ in vacuo at room temperature prior to use. All other compounds are commercially available and were used without further purification.

\section{General procedure for the synthesis and characterization of potassium carboxylate salts (1a-l).}

A $250 \mathrm{~mL}$, two-necked, round-bottomed flask was charged with the carboxylic acid (20.0 $\mathrm{mmol}$ ) and ethanol $(20.0 \mathrm{~mL})$. To this, a solution of potassium tert-butoxide ( $2.24 \mathrm{~g}, 20.0$ mmol) in ethanol $(20.0 \mathrm{~mL})$ was added dropwise over $2 \mathrm{~h}$. After complete addition, the reaction mixture was stirred for another $1 \mathrm{~h}$ at room temperature. A gradual formation of a white precipitate was observed. The resulting solid was collected by filtration washed sequentially with ethanol ( 2 x $10.0 \mathrm{~mL})$ and cold $\left(0{ }^{\circ} \mathrm{C}\right)$ diethyl ether $(10.0 \mathrm{~mL})$, and dried in vacuum to provide the corresponding potassium salts of the carboxylic acids.

The known compounds were identified by comparison of their spectra with those of authentic samples. The unknown compounds were characterized by their spectroscopic data (IR, ${ }^{1} \mathrm{H}$ and ${ }^{13} \mathrm{C}$ NMR, and elemental analysis), which are provided below in order of their entries in the respective tables.

Potassium 5-chloro-2-nitrobenzoate (1e). Compound 1e was prepared following the general procedure starting from 5-chloro-2-nitrobenzoic acid (4.03 g, $20.0 \mathrm{mmol})$ and potassium tertbutoxide (2.24 g, $20.0 \mathrm{mmol}$ ). After filtration, 1e was isolated as a white powder (3.93 g, $82 \%)$. mp $288{ }^{\circ} \mathrm{C}$. IR (KBr): $v=1619,1348,1151,1093,877,742 \mathrm{~cm}^{-1} .{ }^{1} \mathrm{H}$ NMR (400 MHz, $\left.\mathrm{CD}_{3} \mathrm{OD}\right) \delta 7.93(\mathrm{~d}, J=8.6 \mathrm{~Hz}, 1 \mathrm{H}), 7.50-7.59(\mathrm{~m}, 2 \mathrm{H}) \mathrm{ppm} .{ }^{13} \mathrm{C}$ NMR $\left(101 \mathrm{MHz}, \mathrm{CD}_{3} \mathrm{OD}\right)$ $\delta$ 171.9, 146.7, 140.6, 139.7, 130.1, 129.9, 126.8 ppm. Anal. Calcd. for $\mathrm{C}_{7} \mathrm{H}_{3} \mathrm{ClKNO}_{4}$ : C, 35.08; H, 1.26; N, 5.84. Found: C, 35.28; H, 1.41; N, 5.88.

Potassium 2-(methylsulfonyl)benzoate (11). Compound 11 was prepared following the general procedure starting from 2-(methylsulfonyl)benzoic acid (4.00 g, $20.0 \mathrm{mmol}$ ) and potassium tert-butoxide $(2.24 \mathrm{~g}, 20.0 \mathrm{mmol})$. After filtration, 11 was isolated as a white powder (3.81 g, 80\%). mp $258{ }^{\circ} \mathrm{C}$. IR (KBr): $v=1588,1394,1308,1148,1120,966,755$ $\mathrm{cm}^{-1} .{ }^{1} \mathrm{H}$ NMR (400 MHz, CD $\left.3 \mathrm{OD}\right) \delta 7.96$ (dd, $\left.J=7.9,0.9 \mathrm{~Hz}, 1 \mathrm{H}\right), 7.67$ (td, $J=7.5,1.2 \mathrm{~Hz}$, $1 \mathrm{H}), 7.49$ - 7.57 (m, $2 \mathrm{H}$ ), 3.38 (s, $3 \mathrm{H}) \mathrm{ppm} .{ }^{13} \mathrm{C}$ NMR (101 MHz, $\left.\mathrm{CD}_{3} \mathrm{OD}\right) \delta 175.7,144.0$, 137.3, 135.0, 129.7, 129.2, 129.1, 45.0 ppm. Anal. Calcd. for $\mathrm{C}_{8} \mathrm{H}_{7} \mathrm{KO}_{4} \mathrm{~S}$ : C, 40.32; H, 2.96; S, 13.46. Found: C, 40.31; H, 2.83; N, 13.50. 
Potassium 2-(trifluoromethyl)benzoate (1m). Compound $1 \mathbf{m}$ was prepared following the general procedure starting from 2-(trifluoromethyl)benzoic acid (0.95 g, $5.00 \mathrm{mmol}$ ) and potassium tert-butoxide $(0.57 \mathrm{~g}, 5.00 \mathrm{mmol})$. After filtration, $\mathbf{1 m}$ was isolated as a white powder (1.12 g, 96\%). mp $203{ }^{\circ} \mathrm{C}$. IR (KBr): $v=1564,1400,1315,1176,1035,772 \mathrm{~cm}^{-1} .{ }^{1} \mathrm{H}$ NMR (400 MHz, CD $\left.{ }_{3} \mathrm{OD}\right) \delta 7.62$ (d, $\left.J=7.8 \mathrm{~Hz}, 1 \mathrm{H}\right), 7.52-7.59(\mathrm{~m}, 1 \mathrm{H}), 7.49-7.52$ (m, 1 $\mathrm{H}), 7.39-7.45(\mathrm{~m}, 1 \mathrm{H}) \mathrm{ppm} .{ }^{13} \mathrm{C}$ NMR $\left(101 \mathrm{MHz}, \mathrm{CD}_{3} \mathrm{OD}\right) \delta 176.4,142.4\left(\mathrm{q}, J_{C, F}=2.5\right.$ $\mathrm{Hz}$ ), 133.0, 128.9, 128.8, $127\left(\mathrm{q}, J_{C, F}=31.6 \mathrm{~Hz}\right), 126.8$ (q, $\left.J_{C, F}=5.2 \mathrm{~Hz}\right), 125.9\left(\mathrm{q}, J_{C, F}=\right.$ $272.5 \mathrm{~Hz}$ ) ppm. Anal. Calcd. for $\mathrm{C}_{8} \mathrm{H}_{4} \mathrm{~F}_{3} \mathrm{KO}_{2}$ : C, 42.10; H, 1.77. Found: C, 42.22; $\mathrm{H}, 1.91$.

\section{General procedure for the aryl ether synthesis:}

A reaction vessel was charged with the potassium carboxylate (1a-o) $(1.00 \mathrm{mmol})$, silver carbonate (69 mg, $25 \mathrm{~mol} \%$ ), copper(II) acetate (182 mg, $1 \mathrm{mmol}$ ) and the silane (2 a-f) (1.2 mmol). Anhydrous DMF (5 mL) was added to it, the reaction vessel was briefly purged with oxygen, and the resulting mixture was stirred at $145{ }^{\circ} \mathrm{C}$ for $18 \mathrm{~h}$ at ambient oxygen pressure. After cooling to room temperature, the reaction mixture was diluted with diethyl ether (20 $\mathrm{mL})$ and washed with saturated aqueous sodium bicarbonate $(20 \mathrm{~mL})$. The aqueous layer was extracted with diethyl ether ( 3 x $20 \mathrm{~mL}$ ), and the organic layers were washed with water, $5 \mathrm{~N}$ $\mathrm{HCl}$ and brine, dried over $\mathrm{MgSO}_{4}$, filtered, and concentrated in vacuum. The residue was purified by column chromatography (silica gel, $n$-hexane) to yield the corresponding aryl ether (Заa...3oa).

1-Methoxy-2-nitrobenzene (3aa) [CAS: 91-23-6]. Compound 3aa was synthesized following the general procedure starting from potassium 2-nitrobenzoate (205 mg, 1.00 mmol) and tetramethylorthosilicate (777 $\mathrm{mg}, 5.00 \mathrm{mmol})$. After column chromatography $\left(\mathrm{SiO}_{2}\right.$, hexane/diethyl ether 4:1), 3aa was isolated as a yellow liquid (130 mg, 85\%). ${ }^{1} \mathrm{H} \mathrm{NMR}$ $\left(400 \mathrm{MHz}, \mathrm{CDCl}_{3}\right) \delta 7.78-7.81(\mathrm{~m}, 1 \mathrm{H}), 7.50-7.54$ (m, $\left.1 \mathrm{H}\right), 7.07-7.09(\mathrm{~m}, 1 \mathrm{H}), 6.98-$ 7.02 (m, $1 \mathrm{H}), 3.93$ (s, $3 \mathrm{H}) \mathrm{ppm} .{ }^{13} \mathrm{C}$ NMR (101 MHz, $\left.\mathrm{CDCl}_{3}\right) \delta 152.8,139.5,134.2,125.4$,

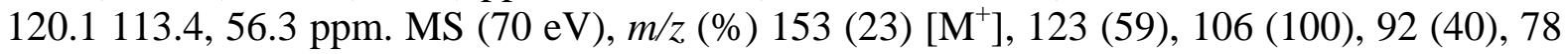
(32), 77 (60), 63 (32), 51 (34)

1-Ethoxy-2-nitrobenzene (3ab) [CAS: 610-67-3]. Compound 3ab was synthesized following the general procedure starting from potassium 2-nitrobenzoate (205 $\mathrm{mg}, 1.00$ mmol) and tetraethylorthosilicate (250 mg, $1.20 \mathrm{mmol})$. After column chromatography $\left(\mathrm{SiO}_{2}\right.$, hexane/diethyl ether 4:1), 3ab was isolated as a yellow liquid (131 mg, 78\%). ${ }^{1} \mathrm{H}$ NMR (400 $\left.\mathrm{MHz}, \mathrm{CDCl}_{3}\right) \delta 7.79(\mathrm{dd}, J=8.1,1.7 \mathrm{~Hz}, 1 \mathrm{H}), 7.46-7.55(\mathrm{~m}, 1 \mathrm{H}), 7.05-7.08(\mathrm{~m}, 1 \mathrm{H})$, $6.98-7.02(\mathrm{~m}, 1 \mathrm{H}), 4.17$ (q, $J=7.0 \mathrm{~Hz}, 2 \mathrm{H}), 1.46$ (t, $J=7.0 \mathrm{~Hz}, 3 \mathrm{H}) \mathrm{ppm} .{ }^{13} \mathrm{C}$ NMR $(101$ $\left.\mathrm{MHz}, \mathrm{CDCl}_{3}\right) \delta 152.2,140.0,133.9,125.4,120.0,114.4,65.2,14.4$ ppm. HRMS (EI-TOF) calcd for $\mathrm{C}_{8} \mathrm{H}_{9} \mathrm{NO}_{3}, 167.0582$, found 167.0583.

1-Nitro-2-propoxybenzene (3ac) [CAS: 3079-53-6]. Compound 3ac was synthesized following the general procedure starting from potassium 2-nitrobenzoate (205 $\mathrm{mg}, 1.00$ mmol) and tetrapropylorthosilicate (317 $\mathrm{mg}, 1.20 \mathrm{mmol}$ ). After column chromatography $\left(\mathrm{SiO}_{2}\right.$, hexane/diethyl ether 4:1), 3ac was isolated as a yellow liquid (153 mg, 84\%). ${ }^{1} \mathrm{H}$ NMR $\left(400 \mathrm{MHz}, \mathrm{CDCl}_{3}\right) \delta 7.80$ (dd, $\left.J=8.1,1.7 \mathrm{~Hz}, 1 \mathrm{H}\right), 7.46-7.52(\mathrm{~m}, 1 \mathrm{H}), 7.05-7.08$ (m, 1 H) $6.97-7.01(\mathrm{~m}, 1 \mathrm{H}), 4.06(\mathrm{t}, J=6.4 \mathrm{~Hz}, 2 \mathrm{H}), 1.83-1.88(\mathrm{~m}, 2 \mathrm{H}), 1.06(\mathrm{t}, J=7.5 \mathrm{~Hz}, 3$ 
H), ppm. ${ }^{13} \mathrm{C}$ NMR $\left(101 \mathrm{MHz}, \mathrm{CDCl}_{3}\right) \delta 152.4,139.9,133.9,125.4,119.9,114.3,70.9,22.3$, 10.3 ppm. HRMS (EI-TOF) calcd for $\mathrm{C}_{9} \mathrm{H}_{11} \mathrm{NO}_{3}, 181.0739$, found 181.0737.

1-Butoxy-2-nitrobenzene (3ad) [CAS: 7252-51-9]. Compound 3ad was synthesized following the general procedure starting from potassium 2-nitrobenzoate (205 mg, 1.00 mmol) and tetrabutylorthosilicate (385 mg, $1.20 \mathrm{mmol})$. After column chromatography $\left(\mathrm{SiO}_{2}\right.$, hexane/diethyl ether 4:1), 3ad was isolated as a yellow liquid (162 mg, 83\%). ${ }^{1} \mathrm{H}$ NMR (400 $\left.\mathrm{MHz}, \mathrm{CDCl}_{3}\right) \delta 7.80$ (dd, $\left.J=8.1,1.7 \mathrm{~Hz}, 1 \mathrm{H}\right), 7.47-7.50(\mathrm{~m}, 1 \mathrm{H}) 7.05-7.08(\mathrm{~m}, 1 \mathrm{H}) 6.96$ - 7.01 (m, $1 \mathrm{H}), 4.09$ (t, $J=6.4$ Hz, $2 \mathrm{H}), 1.76$ - 1.83 (m, 2 H), 1.48 - 1.54 (m, $2 \mathrm{H}), 0.96$ (t, $J$ $=7.3 \mathrm{~Hz}, 3 \mathrm{H}) \mathrm{ppm} .{ }^{13} \mathrm{C} \mathrm{NMR}\left(101 \mathrm{MHz}, \mathrm{CDCl}_{3}\right) \delta 152.4,139.8,133.9,125.3,119.9,114.3$, 69.2, 30.9, 19.0, 13.6 ppm. HRMS (EI-TOF) calcd for $\mathrm{C}_{10} \mathrm{H}_{13} \mathrm{NO}_{3}, 195.0895$, found 195.0895 .

1-Isopropoxy-2-nitrobenzene (3ae) [CAS: 38753-50-3]. Compound 3ae was synthesized following the general procedure starting from potassium 2-nitrobenzoate (205 mg, 1.00 $\mathrm{mmol}$ ) and tetraisopropylorthosilicate (317 $\mathrm{mg}, 1.20 \mathrm{mmol}$ ). After column chromatography $\left(\mathrm{SiO}_{2}\right.$, hexane/diethyl ether 4:1), 3ae was isolated as a pale yellow liquid (146 mg, 81\%). ${ }^{1} \mathrm{H}$ NMR (400 MHz, $\left.\mathrm{CDCl}_{3}\right) \delta 7.76(\mathrm{dd}, J=8.1,1.7 \mathrm{~Hz}, 1 \mathrm{H}), 7.46-7.51(\mathrm{~m}, 1 \mathrm{H}), 7.08$ (d, $J=$ $8.3 \mathrm{~Hz}, 1 \mathrm{H}), 6.97-7.01(\mathrm{~m}, 1 \mathrm{H}), 4.65-4.71(\mathrm{~m}, 1 \mathrm{H}), 1.39$ (d, $J=6.1 \mathrm{~Hz}, 6 \mathrm{H}) \mathrm{ppm} .{ }^{13} \mathrm{C}$ NMR (101 MHz, $\left.\mathrm{CDCl}_{3}\right) \delta 151.2,141.1,133.6,125.4,120.0,116.0,77.3,76.7,72.5,21.8$ ppm. HRMS (EI-TOF) calcd for $\mathrm{C}_{9} \mathrm{H}_{11} \mathrm{NO}_{3}, 181.0739$, found 181.0744.

1-Nitro-2-phenoxybenzene (3af) [CAS: 2216-12-8]. Compound 3af was synthesized following the general procedure starting from potassium 2-nitrobenzoate (205 $\mathrm{mg}, 1.00$ mmol) and triphenylborate (348 mg, $1.20 \mathrm{mmol})$. After column chromatography $\left(\mathrm{SiO}_{2}\right.$, hexane/diethyl ether 4:1), 3af was isolated as a pale yellow liquid (103 mg, 48\%). ${ }^{1} \mathrm{H}$ NMR $\left(400 \mathrm{MHz}, \mathrm{CDCl}_{3}\right) \delta 7.95$ (dd, $\left.J=8.2,1.6 \mathrm{~Hz}, 1 \mathrm{H}\right), 7.48-7.53(\mathrm{~m}, 1 \mathrm{H}), 7.37-7.41$ (m, 2 $\mathrm{H}), 7.14-7.24(\mathrm{~m}, 2 \mathrm{H}), 6.98-7.10(\mathrm{~m}, 3 \mathrm{H}) \mathrm{ppm} .{ }^{13} \mathrm{C} \mathrm{NMR}\left(101 \mathrm{MHz}, \mathrm{CDCl}_{3}\right) \delta 155.6$, 150.6, 141.2, 134.1, 130.0 (2C), 125.6, 124.5, 123.0, 120.3, 119.1 (2C) ppm. HRMS (EITOF) calcd for $\mathrm{C}_{12} \mathrm{H}_{9} \mathrm{NO}_{3}$, 215.0582, found 215.0576.

2-Ethoxy-1-methyl-3-nitrobenzene (3bb) [CAS: 1208074-91-2]. Compound 3bb was synthesized following the general procedure starting from potassium 2-methyl-6nitrobenzoate (219 mg, $1.00 \mathrm{mmol}$ ) and tetraethylorthosilicate (250 mg, $1.20 \mathrm{mmol}$ ). After column chromatography $\left(\mathrm{SiO}_{2}\right.$, hexane/diethyl ether 4:1), 3bb was isolated as a pale yellow liquid (141 mg, 78\%). ${ }^{1} \mathrm{H}$ NMR (400 MHz, $\left.\mathrm{CDCl}_{3}\right) \delta 7.62$ (dd, $J=8.0,1.2 \mathrm{~Hz}, 1 \mathrm{H}$ ), 7.40 7.42 (m, 1 H), 7.09 (t, $J=7.9$ Hz, 1 H), 4.04 (q, $J=7.0$ Hz, 2 H), 2.36 (s, 3 H), 1.43 (t, $J=$ $7.0 \mathrm{~Hz}, 3 \mathrm{H}) \mathrm{ppm} .{ }^{13} \mathrm{C}$ NMR $\left(101 \mathrm{MHz}, \mathrm{CDCl}_{3}\right) \delta 150.7,144.5,135.4,134.6,123.5,122.8$, 70.4, 16.2, 15.5 ppm. HRMS (EI-TOF) calcd for $\mathrm{C}_{9} \mathrm{H}_{11} \mathrm{NO}_{3}, 181.0739$, found 181.0742.

2-Ethoxy-4-methyl-1-nitrobenzene (3cb) [CAS: 102871-93-2]. Compound 3cb was synthesized following the general procedure starting from potassium 5-methyl-2nitrobenzoate (219 mg, $1.00 \mathrm{mmol}$ ) and tetraethylorthosilicate (250 mg, $1.20 \mathrm{mmol}$ ). After column chromatography $\left(\mathrm{SiO}_{2}\right.$, hexane/diethyl ether 4:1), 3cb was isolated as a pale yellow liquid (149 mg, 82\%). ${ }^{1} \mathrm{H}$ NMR (400 MHz, $\left.\mathrm{CDCl}_{3}\right) \delta 7.74(\mathrm{~d}, J=8.2 \mathrm{~Hz}, 1 \mathrm{H}), 6.85(\mathrm{~s}, 1 \mathrm{H})$, $6.78(\mathrm{~d}, J=8.2 \mathrm{~Hz}, 1 \mathrm{H}), 4.15$ (q, $J=7.0 \mathrm{~Hz}, 2 \mathrm{H}), 2.39(\mathrm{~s}, 3 \mathrm{H}), 1.46$ (t, $J=7.0 \mathrm{~Hz}, 3 \mathrm{H})$ ppm. ${ }^{13} \mathrm{C}$ NMR (101 MHz, $\left.\mathrm{CDCl}_{3}\right) \delta 152.4,145.6,137.5,125.6,120.7,114.9,65.2,21.8$, 14.5 ppm. HRMS (EI-TOF) calcd for $\mathrm{C}_{9} \mathrm{H}_{11} \mathrm{NO}_{3}, 181.0739$, found 181.0746. 
1-Ethoxy-3-methyl-2-nitrobenzene (3db) [CAS: 342044-61-5]. Compound 3db was synthesized following the general procedure starting from potassium 3-methyl-2nitrobenzoate (219 mg, $1.00 \mathrm{mmol}$ ) and tetraethylorthosilicate (250 mg, $1.20 \mathrm{mmol}$ ). After column chromatography $\left(\mathrm{SiO}_{2}\right.$, hexane/diethyl ether 4:1), 3db was isolated as a pale yellow liquid (132 mg, 73\%). ${ }^{1} \mathrm{H}$ NMR (400 MHz, $\left.\mathrm{CDCl}_{3}\right) \delta 7.24-7.28(\mathrm{~m}, 1 \mathrm{H}), 6.73-6.93$ (m, 2 $\mathrm{H}), 4.10(\mathrm{q}, J=6.9 \mathrm{~Hz}, 2 \mathrm{H}), 2.29(\mathrm{~s}, 3 \mathrm{H}), 1.38(\mathrm{t}, J=6.9 \mathrm{~Hz}, 3 \mathrm{H}) \mathrm{ppm} .{ }^{13} \mathrm{C}$ NMR $(101$ $\left.\mathrm{MHz} \mathrm{CDCl}_{3}\right) \delta 150.0,142.3,130.7,130.5,122.3,110.9,65.0,16.8,14.4$ ppm. HRMS (EITOF) calcd for $\mathrm{C}_{9} \mathrm{H}_{11} \mathrm{NO}_{3}, 181.0739$, found 181.0743.

4-Chloro-2-ethoxy-1-nitrobenzene (3eb) [CAS: 29604-25-9]. Compound 3eb was synthesized following the general procedure starting from potassium 5-chloro-2nitrobenzoate (240 mg, $1.00 \mathrm{mmol}$ ) and tetraethylorthosilicate (250 mg, $1.20 \mathrm{mmol}$ ). After column chromatography $\left(\mathrm{SiO}_{2}\right.$, hexane/diethyl ether 4:1), 3eb was isolated as a pale yellow solid (137 mg, 68\%) mp $63{ }^{\circ} \mathrm{C} .{ }^{1} \mathrm{H}$ NMR (400 MHz, $\left.\mathrm{CDCl}_{3}\right) \delta 7.81$ (d, $\left.J=8.6 \mathrm{~Hz}, 1 \mathrm{H}\right), 7.06$ (d, $J=2.0 \mathrm{~Hz}, 1 \mathrm{H}), 6.08-7.01(\mathrm{~m}, 1 \mathrm{H}), 4.18$ (q, $J=7.0 \mathrm{~Hz}, 2 \mathrm{H}), 1.49$ (t, $J=7.0 \mathrm{~Hz}, 3 \mathrm{H}$ ) ppm. ${ }^{13} \mathrm{C}$ NMR (101 MHz, $\left.\mathrm{CDCl}_{3}\right) \delta 153.0,140.1,138.3,126.7,120.3,114.9,65.8,14.4$ ppm. Anal. Calcd. for $\mathrm{C}_{8} \mathrm{H}_{8} \mathrm{ClNO}_{3}$ : C, 47.66; H, 4.00; N, 6.95. Found: C, 47.76; H, 4.12; N, 6.81.

2-Ethoxy-4-methoxy-1-nitrobenzene (3fb) [CAS: 151929-97-4]. Compound 3fb was synthesized following the general procedure starting from potassium 5-methoxy-2nitrobenzoate (235 mg, $1.00 \mathrm{mmol}$ ) and tetraethylorthosilicate (250 mg, $1.20 \mathrm{mmol}$ ). After column chromatography $\left(\mathrm{SiO}_{2}\right.$, hexane/diethyl ether 4:1), 3fb was isolated as a pale yellow liquid (166 mg, 84\%). ${ }^{1} \mathrm{H}$ NMR (400 MHz, $\left.\mathrm{CDCl}_{3}\right) \delta 7.92$ (d, $J=8.8 \mathrm{~Hz}, 1 \mathrm{H}$ ), $6.45-6.49$ (m, $2 \mathrm{H}), 4.12$ (q, $J=6.9 \mathrm{~Hz}, 2 \mathrm{H}), 3.85(\mathrm{~s}, 3 \mathrm{H}), 1.46$ (t, $J=7.0 \mathrm{~Hz}, 3 \mathrm{H}) \mathrm{ppm} .{ }^{13} \mathrm{C}$ NMR $\left(101 \mathrm{MHz}, \mathrm{CDCl}_{3}\right) \delta 164.5,154.9,133.0,128.1,104.7,100.2,65.2,55.8,14.4$ ppm. HRMS (EI-TOF) calcd for $\mathrm{C}_{9} \mathrm{H}_{11} \mathrm{NO}_{4}, 197.0688$, found 197.0692 .

1,2-Dimethoxybenzene (3ga) [CAS: 91-16-7]. Compound 3ga was synthesized following the general procedure starting from potassium 2-methoxybenzoate $(190 \mathrm{mg}, 1.00 \mathrm{mmol})$ and tetramethylorthosilicate $(777 \mathrm{mg}, 5.00 \mathrm{mmol})$. After column chromatography $\left(\mathrm{SiO}_{2}\right.$, hexane/diethyl ether 4:1), 3ga was isolated as a colourless liquid (108 mg, 78\%). ${ }^{1} \mathrm{H}$ NMR $\left(400 \mathrm{MHz}, \mathrm{CDCl}_{3}\right) \delta 6.87-6.94(\mathrm{~m}, 4 \mathrm{H}), 3.88(\mathrm{~s}, 6 \mathrm{H}) \mathrm{ppm} .{ }^{13} \mathrm{C}$ NMR $\left(101 \mathrm{MHz}, \mathrm{CDCl}_{3}\right) \delta$ 148.9 (2C), 120.7 (2C), 111.2 (2C), 55.6 (2C) ppm. MS (70 eV), m/z (\%) 138 (100) $\left[\mathrm{M}^{+}\right]$, 109 (47), 108 (16), 95 (22), 78 (32), 65 (22), 63 (14).

1,2,3-Trimethoxybenzene (3ha) [CAS: 634-36-6]. Compound 3ha was synthesized following the general procedure starting from potassium 2,6-dimethoxybenzoate $(220 \mathrm{mg}$, $1.00 \mathrm{mmol}$ ) and tetramethylorthosilicate (777 mg, $5.00 \mathrm{mmol}$ ). After column chromatography $\left(\mathrm{SiO}_{2}\right.$, hexane/ethyl acetate 4:1), 3ha was isolated as a colourless solid (133 mg, 79\%). mp 44 ${ }^{\circ} \mathrm{C}$. ${ }^{1} \mathrm{H}$ NMR $\left(400 \mathrm{MHz}, \mathrm{CDCl}_{3}\right) \delta 6.99(\mathrm{t}, J=8.4 \mathrm{~Hz}, 1 \mathrm{H}), 6.58$ (d, $J=8.3 \mathrm{~Hz}, 2 \mathrm{H}$ ), 3.85 (s, $9 \mathrm{H})$ ppm. ${ }^{13} \mathrm{C}$ NMR (101 MHz, $\left.\mathrm{CDCl}_{3}\right) \delta 153.4$ (2C), 138.0, 123.5, 105.1 (2C), 60.7, 55.9 (2C) ppm. Anal. Calcd. for $\mathrm{C}_{9} \mathrm{H}_{12} \mathrm{O}_{3}$ : C, 64.27; H, 7.19. Found: C, 64.05; H: 7.06.

1-Methoxy-2-(methylsulfonyl)benzene (3ia) [CAS: 13736-79-3]. Compound 3ia was synthesized following the general procedure starting from potassium 2(methylsulfonyl)benzoate (238 mg, $1.00 \mathrm{mmol}$ ) and tetramethylorthosilicate (777 mg, 5.00 mmol). After column chromatography $\left(\mathrm{SiO}_{2}\right.$, hexane/ethyl acetate 4:1), 3ia was isolated as a colourless solid (136 mg, 73\%). mp $93{ }^{\circ} \mathrm{C}$. ${ }^{1} \mathrm{H}$ NMR (400 MHz, $\left.\mathrm{CDCl}_{3}\right) \delta$ 7.92-7.95 (m, 1 
H), 7.56 - 7.60 (m, $1 \mathrm{H}), 7.04$ - 7.10 (m, $2 \mathrm{H}), 3.98$ (s, $3 \mathrm{H}), 3.19$ (s, $3 \mathrm{H}) \mathrm{ppm} .{ }^{13} \mathrm{C}$ NMR (101 $\left.\mathrm{MHz}, \mathrm{CDCl}_{3}\right) \delta 157.1,135.5,129.5,128.2,120.5,112.2,56.2,42.8 \mathrm{ppm} . \mathrm{MS}(70 \mathrm{eV}), \mathrm{m} / \mathrm{z}$ (\%) 186 (55) [M ], 169 (23), 149 (10), 123 (10), 105 (33), 77 (100).

2-Methoxy- $N, N$-dimethylaniline (3ja) [CAS: 700-75-4]. Compound 3 ja was synthesized following the general procedure starting from potassium 2-(dimethylamino)benzoate (212 $\mathrm{mg}, 1.00 \mathrm{mmol})$ and tetramethylorthosilicate $(777 \mathrm{mg}, 5.00 \mathrm{mmol})$. After column chromatography $\left(\mathrm{SiO}_{2}\right.$, hexane/diethyl ether 4:1), 3ja was isolated as a colourless liquid (83 mg, 55\%). ${ }^{1} \mathrm{H}$ NMR (400 MHz, $\left.\mathrm{CDCl}_{3}\right) \delta 6.84-6.99$ (m, $\left.4 \mathrm{H}\right), 3.90$ (s, $\left.3 \mathrm{H}\right), 2.80$ (s, $\left.6 \mathrm{H}\right)$ ppm. ${ }^{13} \mathrm{C}$ NMR (101 MHz, $\left.\mathrm{CDCl}_{3}\right) \delta 152.4,142.4,122.4,120.7,118.1,110.9,55.3,43.3$ ppm. MS (70 eV), m/z (\%) 151 (100) [M+1, 136 (74), 108 (32), 65 (28).

1-Chloro-2-ethoxy-4-nitrobenzene (3kb) [CAS: 102236-22-6]. Compound 3kb was synthesized following the general procedure starting from potassium 2-chloro-5nitrobenzoate (250 mg, $1.00 \mathrm{mmol}$ ) and tetraethylorthosilicate (250 mg, $1.20 \mathrm{mmol})$. After column chromatography $\left(\mathrm{SiO}_{2}\right.$, hexane/diethyl ether 4:1), 3kb was isolated as a colourless solid (117 mg, 58\%) mp $64{ }^{\circ} \mathrm{C} .{ }^{1} \mathrm{H}$ NMR $\left(400 \mathrm{MHz}, \mathrm{CDCl}_{3}\right) \delta 7.77-7.81(\mathrm{~m}, 2 \mathrm{H}) 7.52(\mathrm{~d}, J$ $=8.6 \mathrm{~Hz}, 1 \mathrm{H}), 4.22(\mathrm{q}, J=7.1 \mathrm{~Hz}, 2 \mathrm{H}), 1.54(\mathrm{t}, J=8.0 \mathrm{~Hz}, 3 \mathrm{H}) \mathrm{ppm} .{ }^{13} \mathrm{C}$ NMR $(101 \mathrm{MHz}$, $\left.\mathrm{CDCl}_{3}\right) \delta 154.8,147.2,130.4,130.2,116.0,107.6,65.4,14.4 \mathrm{ppm}$. MS (70 eV), $\mathrm{m} / \mathrm{z}(\%) 201$ (38) $\left[\mathrm{M}^{+}\right], 173$ (93), 115 (34), 63 (35), 44 (43), 43 (64), 40 (100).

1-Butoxy-2-fluorobenzene (3ld) [CAS: 20781-61-7]. Compound 3ld was synthesized following the general procedure starting from potassium 2-fluorobenzoate (178 mg, 1.00 mmol) and tetrabutylorthosilicate (397 mg, $1.20 \mathrm{mmol})$. After column chromatography $\left(\mathrm{SiO}_{2}\right.$, hexane/diethyl ether 8:1), 3ld was isolated as a colourless liquid (123 mg, 73\%). ${ }^{1} \mathrm{H}$ NMR $\left(400 \mathrm{MHz}, \mathrm{CDCl}_{3}\right) \delta 7.05-7.12(\mathrm{~m}, 2 \mathrm{H}), 6.94-7.01(\mathrm{~m}, 1 \mathrm{H}), 6.89-6.91(\mathrm{~m}, 1 \mathrm{H}), 4.06(\mathrm{t}$, $J=6.6 \mathrm{~Hz}, 2 \mathrm{H}), 1.80-1.87(\mathrm{~m}, 2 \mathrm{H}), 1.50-1.59(\mathrm{~m}, 2 \mathrm{H}), 1.02(\mathrm{t}, J=7.3 \mathrm{~Hz}, 3 \mathrm{H}) \mathrm{ppm} .{ }^{13} \mathrm{C}$ NMR (101 MHz, $\left.\mathrm{CDCl}_{3}\right) \delta 152.8\left(\mathrm{~d}, J_{C, F}=246.4 \mathrm{~Hz}\right), 147.2\left(\mathrm{~d}, J_{C, F}=11.1 \mathrm{~Hz}\right), 124.2,120.7$, 116.1, 114.8, 69.0, 31.2, 19.1, 13.7 ppm. HRMS (EI-TOF) calcd for $\mathrm{C}_{10} \mathrm{H}_{13} \mathrm{FO}, 168.0950$, found 168.0951.

1-Methoxy-2-trifluoromethylbenzene (3ma) [CAS: 395-48-28]. Compound 3ma was synthesized following the general procedure starting from potassium 2trifluoromethylbenzoate $(238 \mathrm{mg}, 1.00 \mathrm{mmol}$ ) and tetramethylorthosilicate (777 $\mathrm{mg}, 5.00$ mmol). After column chromatography $\left(\mathrm{SiO}_{2}\right.$, hexane/diethyl ether 8:1), 3ma was isolated as a colourless liquid (81 mg, 46\%). ${ }^{1} \mathrm{H}$ NMR (400 MHz, $\left.\mathrm{CDCl}_{3}\right) \delta 7.61$ (d, $\left.J=7.6 \mathrm{~Hz}, 1 \mathrm{H}\right), 7.52$ (t, $J=7.7 \mathrm{~Hz}, 1 \mathrm{H}), 7.00-7.05$ (m, $2 \mathrm{H}), 3.92$ (s, $3 \mathrm{H}) \mathrm{ppm} .{ }^{13} \mathrm{C}$ NMR $\left(101 \mathrm{MHz}, \mathrm{CDCl}_{3}\right) \delta$ $157.5\left(\mathrm{q}, J_{C, F}=1.9 \mathrm{~Hz}\right), 133.3,127.0\left(\mathrm{q}, J_{C, F}=5.6 \mathrm{~Hz}\right), 123.8\left(\mathrm{q}, J_{C, F}=272.8 \mathrm{~Hz}\right), 119.3$, $118.1\left(\mathrm{q}, J_{C, F}=31.4 \mathrm{~Hz}\right), 111.9,55.7 \mathrm{ppm}$. MS (70 eV), m/z (\%) $176(100)\left[\mathrm{M}^{+}\right], 146$ (20), 145 (15), 133 (22), 127 (19), 114 (20), 113 (28).

2-Methoxy-1,1'-biphenyl (3na) [CAS: 86-26-0]. Compound 3na was synthesized following the general procedure starting from potassium (1,1'-biphenyl)-2-carboxylate (246 mg, 1.00 $\mathrm{mmol}$ ) and tetramethylorthosilicate (777 $\mathrm{mg}, 5.00 \mathrm{mmol})$. After column chromatography $\left(\mathrm{SiO}_{2}\right.$, hexane/diethyl ether 4:1), 3na was isolated as a colourless liquid (102 mg, $\left.55 \%\right) .{ }^{1} \mathrm{H}$ NMR (400 MHz, $\left.\mathrm{CDCl}_{3}\right) \delta 7.57$ - 7.60 (m, $\left.2 \mathrm{H}\right), 7.44$ - $7.48(\mathrm{~m}, 2 \mathrm{H}), 7.35$ - $7.39(\mathrm{~m}, 3 \mathrm{H})$, 7.02 - 7.08 (m, $2 \mathrm{H}), 3.85$ (s, $3 \mathrm{H}) \mathrm{ppm} .{ }^{13} \mathrm{C}$ NMR (101 MHz, $\left.\mathrm{CDCl}_{3}\right) \delta 156.4,138.5,130.9$, 130.7, 129.5 (2C), 128.6, 128.0, 126.9, 120.8 (2C), 111.2, 55.5 ppm. HRMS (EI-TOF) calcd for $\mathrm{C}_{14} \mathrm{H}_{12} \mathrm{O}_{2}, 212.0837$, found 212.0845 . 
(2-Methoxyphenyl)(phenyl)methanone (3oa) [CAS: 2553-04-0]. Compound 3oa was synthesized following the general procedure starting from potassium 2-benzoylbenzoate (264 $\mathrm{mg}, 1.00 \mathrm{mmol})$ and tetramethylorthosilicate $(777 \mathrm{mg}, 5.00 \mathrm{mmol})$. After column chromatography $\left(\mathrm{SiO}_{2}\right.$, hexane/diethyl ether 5:1), 3oa was isolated as a colourless liquid (136 mg, 64\%). ${ }^{1} \mathrm{H}$ NMR (400 MHz, $\left.\mathrm{CDCl}_{3}\right) \delta 7.81-7.84$ (m, $2 \mathrm{H}$ ), 7.55 (d, $\left.J=7.4 \mathrm{~Hz}, 1 \mathrm{H}\right), 7.42$ - 7.50 (m, $3 \mathrm{H}), 7.36$ - 7.38 (m, $1 \mathrm{H}), 6.99$ - 7.07 (m, $2 \mathrm{H}), 3.73$ (s, $3 \mathrm{H}) \mathrm{ppm} .{ }^{13} \mathrm{C}$ NMR $(101$ $\left.\mathrm{MHz}, \mathrm{CDCl}_{3}\right) \delta 196.4,157.3,137.7,132.9,131.8,129.8,129.5,128.8,128.2$ (3C), 120.4, 111.4, 55.5 ppm. HRMS (EI-TOF) calcd for $\mathrm{C}_{13} \mathrm{H}_{12} \mathrm{O}$, 184.0888, found 184.0887.

(3-Methoxyphenyl)(phenyl)methanone (m-3oa) [6136-67-0]. Compound 3oa' was synthesized following the general procedure starting from potassium 2-benzoylbenzoate (264 $\mathrm{mg}, 1.00 \mathrm{mmol})$ and tetramethylorthosilicate $(777 \mathrm{mg}, 5.00 \mathrm{mmol})$. After column chromatography $\left(\mathrm{SiO}_{2}\right.$, hexane/diethyl ether 5:1), 3oa' was isolated as a colourless liquid (62 mg, 29\%). ${ }^{1} \mathrm{H}$ NMR (400 MHz, $\left.\mathrm{CDCl}_{3}\right) \delta 7.81-7.83(\mathrm{~m}, 2 \mathrm{H}), 7.62(\mathrm{~m}, 1 \mathrm{H}), 7.49-7.53$ (m, 2H), 7.39 - $7.41(\mathrm{~m}, 3 \mathrm{H}), 7.13-7.16(\mathrm{~m}, 1 \mathrm{H}), 3.87$ (s, 3H) ppm. ${ }^{13} \mathrm{C}$ NMR (101 MHz, CDCl $\left.{ }_{3}\right)$ $\delta$ 196.5, 159.5, 138.9, 137.6, 132.4, 130.0 (2C), 129.2, 128.2 (2C), 122.9, 118.9, 114.3, 55.5 ppm. HRMS (EI-TOF) calcd for $\mathrm{C}_{13} \mathrm{H}_{12} \mathrm{O}, 184.0888$, found 184.0889.

1,3-Dimethoxybenzene (7) [151-10-0]. A $10 \mathrm{~mL}$ microwave vial was charged with potassium 4-methoxybenzoate (190 $\mathrm{mg}, 1.00 \mathrm{mmol}$ ), silver carbonate (276 mg, $1 \mathrm{mmol}$ ), copper(II) bromide (226 mg, $1 \mathrm{mmol}$ ). Dry DMF (5 mL) and trimethylborate (579 $\mu \mathrm{L}, 5.00$ mmol) were added via syringe. The resulting mixture was submitted to microwave irradiation at $170{ }^{\circ} \mathrm{C}$ for $40 \mathrm{~min}$ at a maximum power of $150 \mathrm{~W}$ (steady state power: $90 \mathrm{~W}$ ). The sample was air-jet cooled to room temperature every 10 minutes, and GC-samples were taken. The maximum pressure detected during the reaction was 8 bar. After column chromatography $\left(\mathrm{SiO}_{2}\right.$, hexane/diethyl ether 5:1), 7 was isolated as a colourless liquid (57 mg, 41\%). ${ }^{1} \mathrm{H} \mathrm{NMR}$ $\left(400 \mathrm{MHz}, \mathrm{CDCl}_{3}\right) \delta 7.18-7.27(\mathrm{~m}, 1 \mathrm{H}), 6.48-6.54(\mathrm{~m}, 3 \mathrm{H}), 3.81(\mathrm{~s}, 6 \mathrm{H}) \mathrm{ppm} .{ }^{13} \mathrm{C} \mathrm{NMR}$ (101 MHz, $\left.\mathrm{CDCl}_{3}\right) \delta 160.8$ (2C), 129.9, 106.1 (2C), 100.4, 55.3 (2C) ppm. MS (70 eV), m/z (\%) 138 (100) [M $\left.{ }^{+}\right], 109$ (45), 108 (16), 95 (21), 78 (33), 65 (22), 40 (18). 
Potassium 2-nitrobenzoic acid (1a)

${ }^{1} \mathrm{H}$ NMR, $400 \mathrm{MHz}, \mathrm{CD}_{3} \mathrm{OD}$

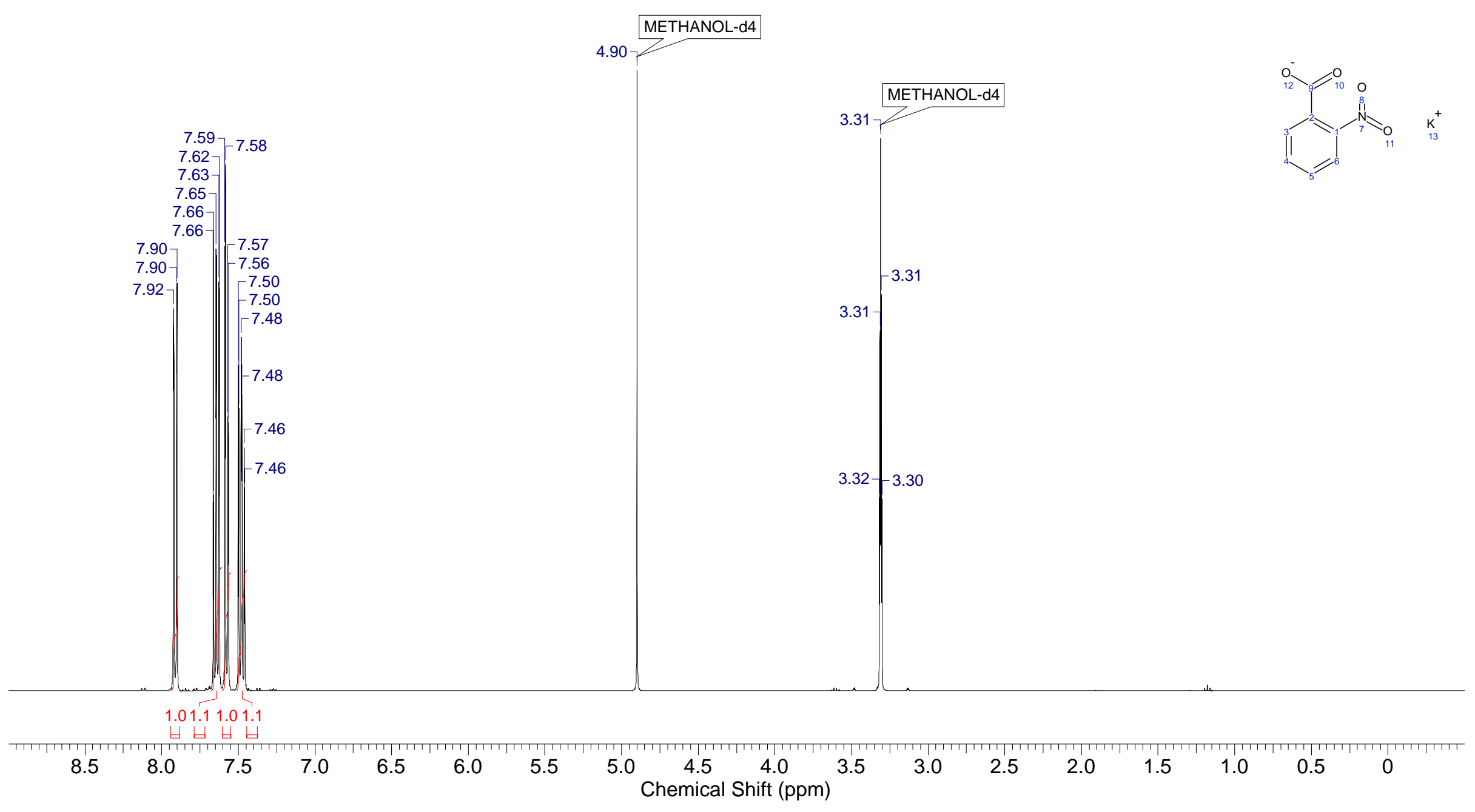


Potassium 2-nitrobenzoate(1a)

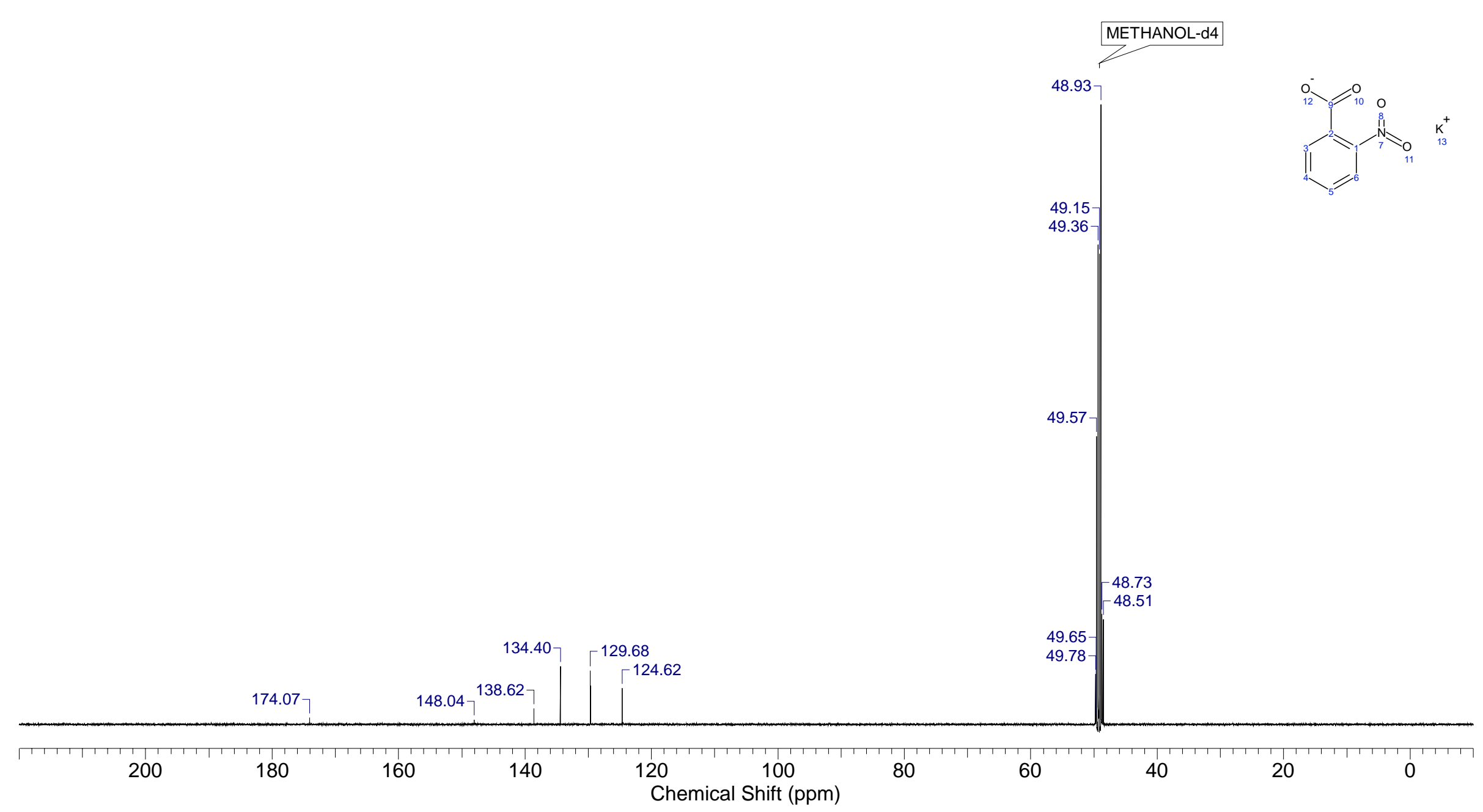


Potassium 2-methyl-6-nitrobenzoate (1b)

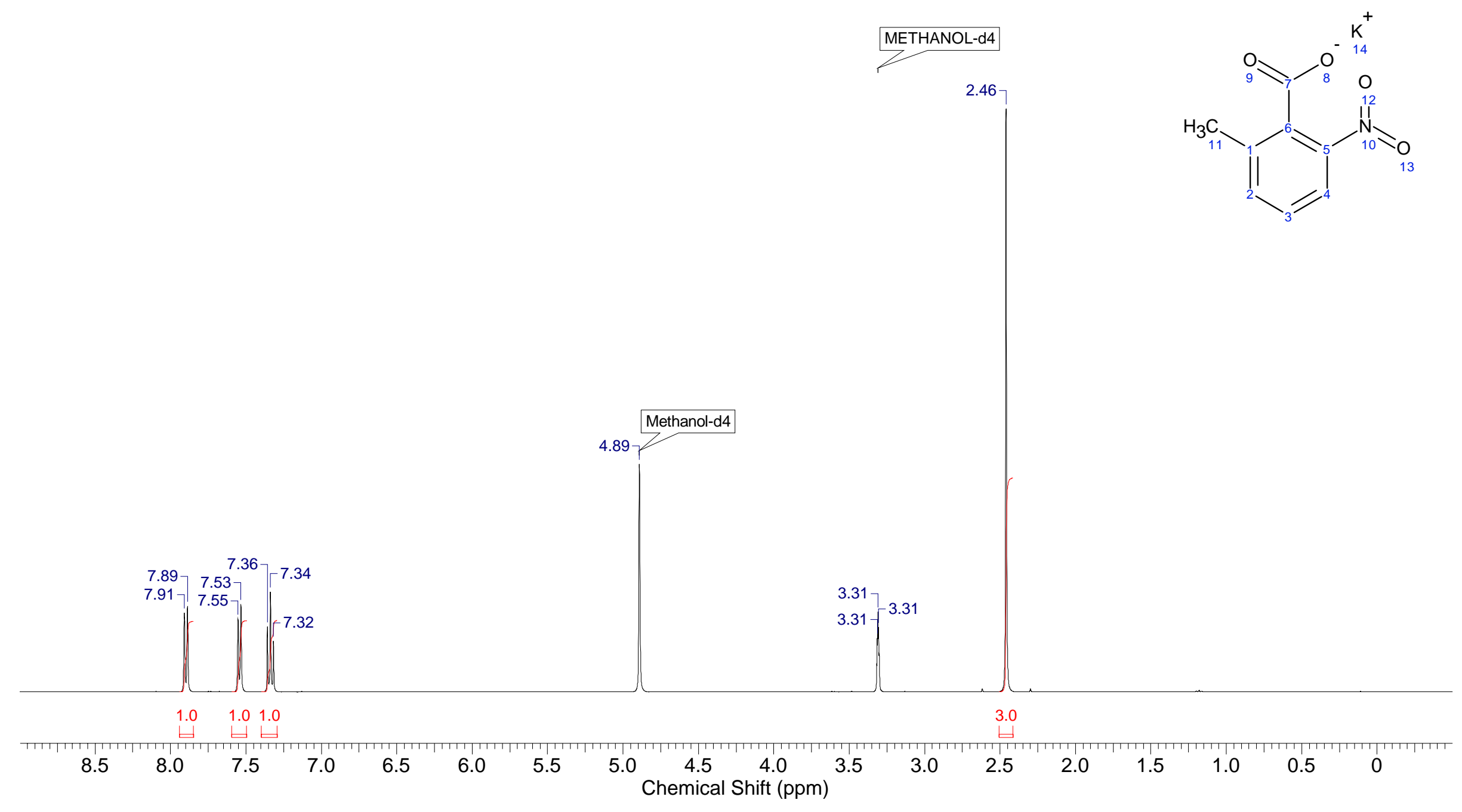


Potassium 2-methyl-6-nitrobenzoate (1b)

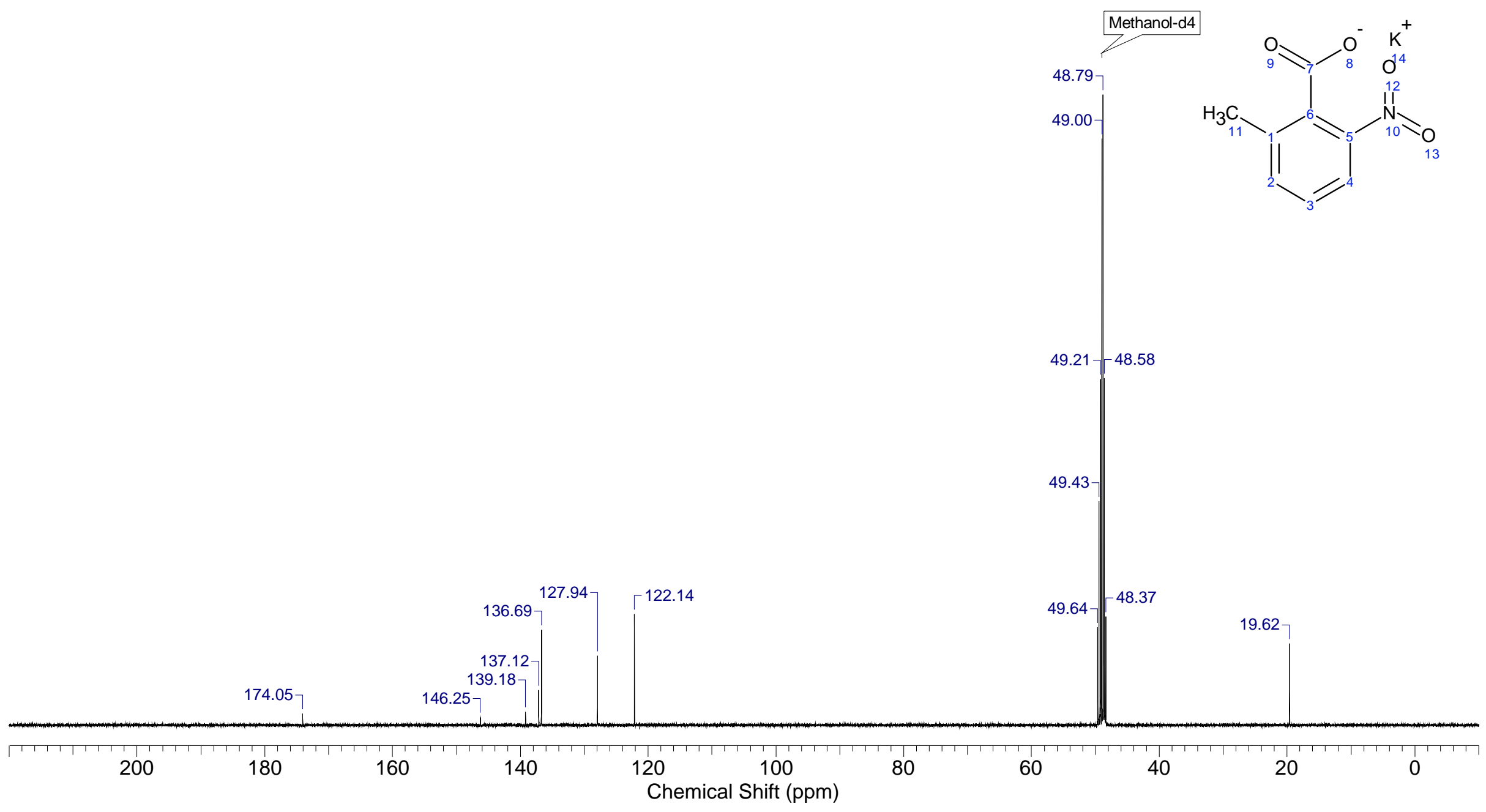


Potassium 5-methyl-2-nitrobenzoate (1c)

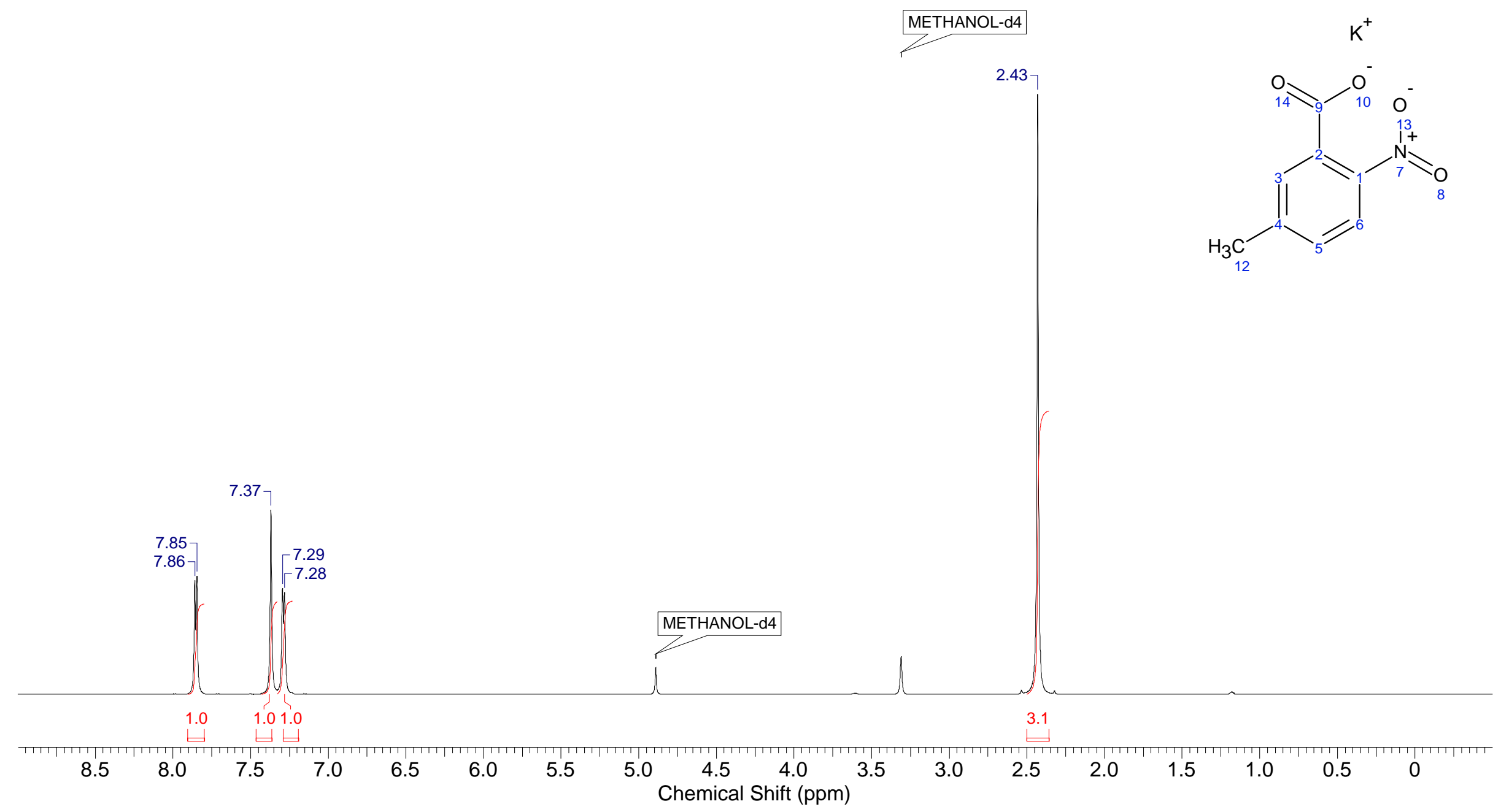


Potassium 5-methyl-2-nitrobenzoate (1c)

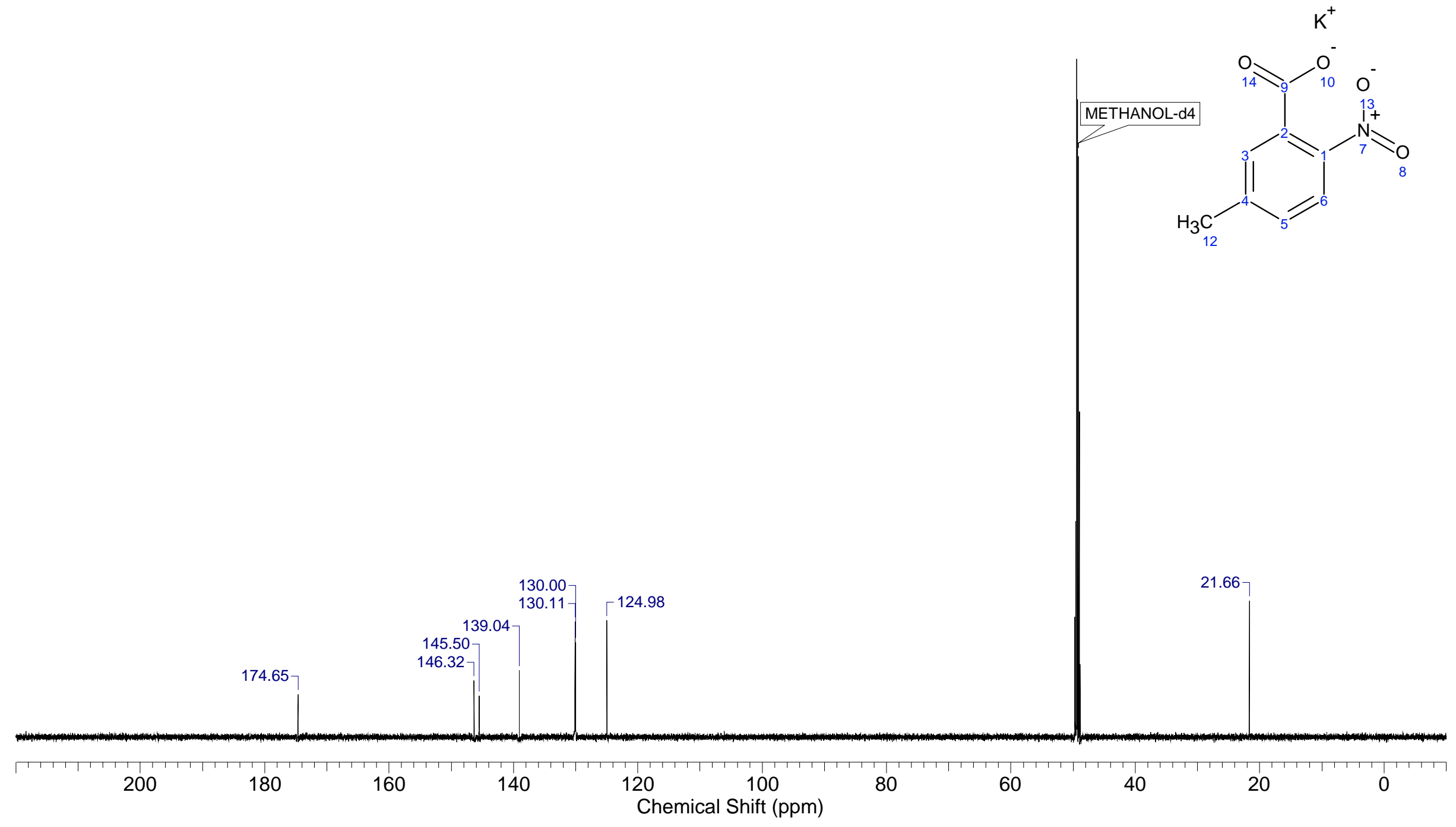


Potassium 3-methyl-2-nitrobenzoate (1d)

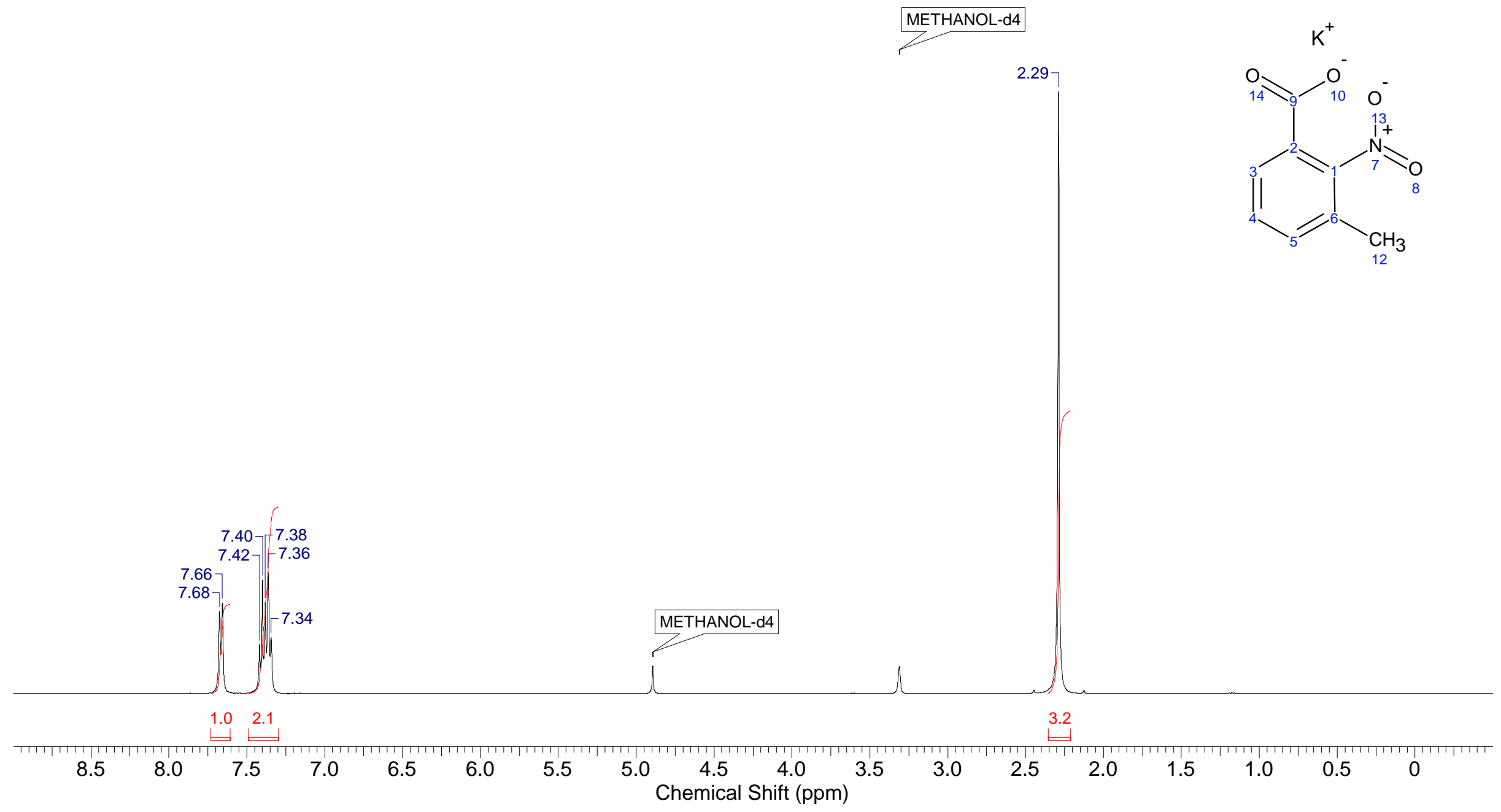


Potassium 3-methyl-2-nitrobenzoate (1d)

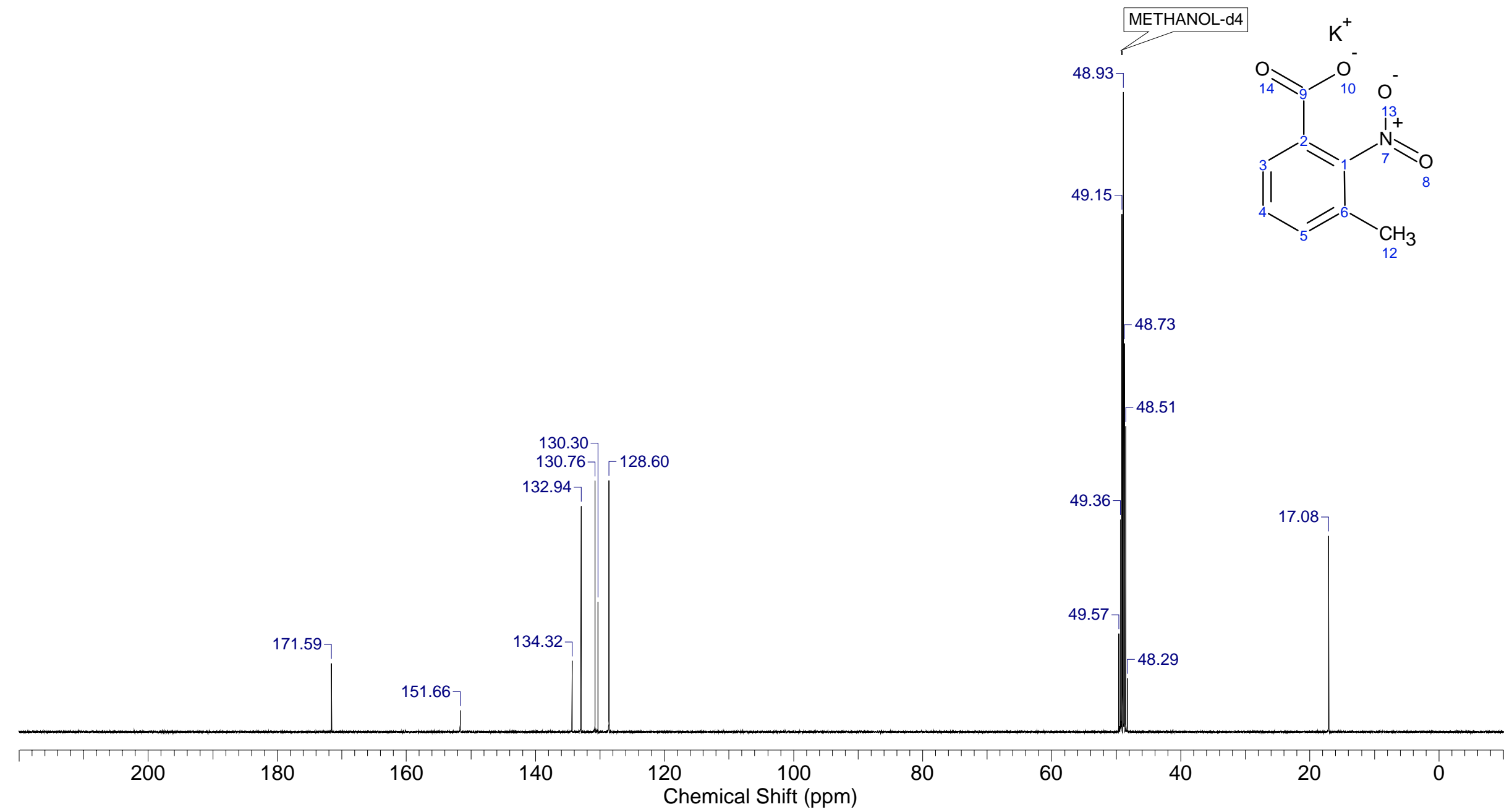


Potassium 5-chloro-2-nitrobenzoate (1e)

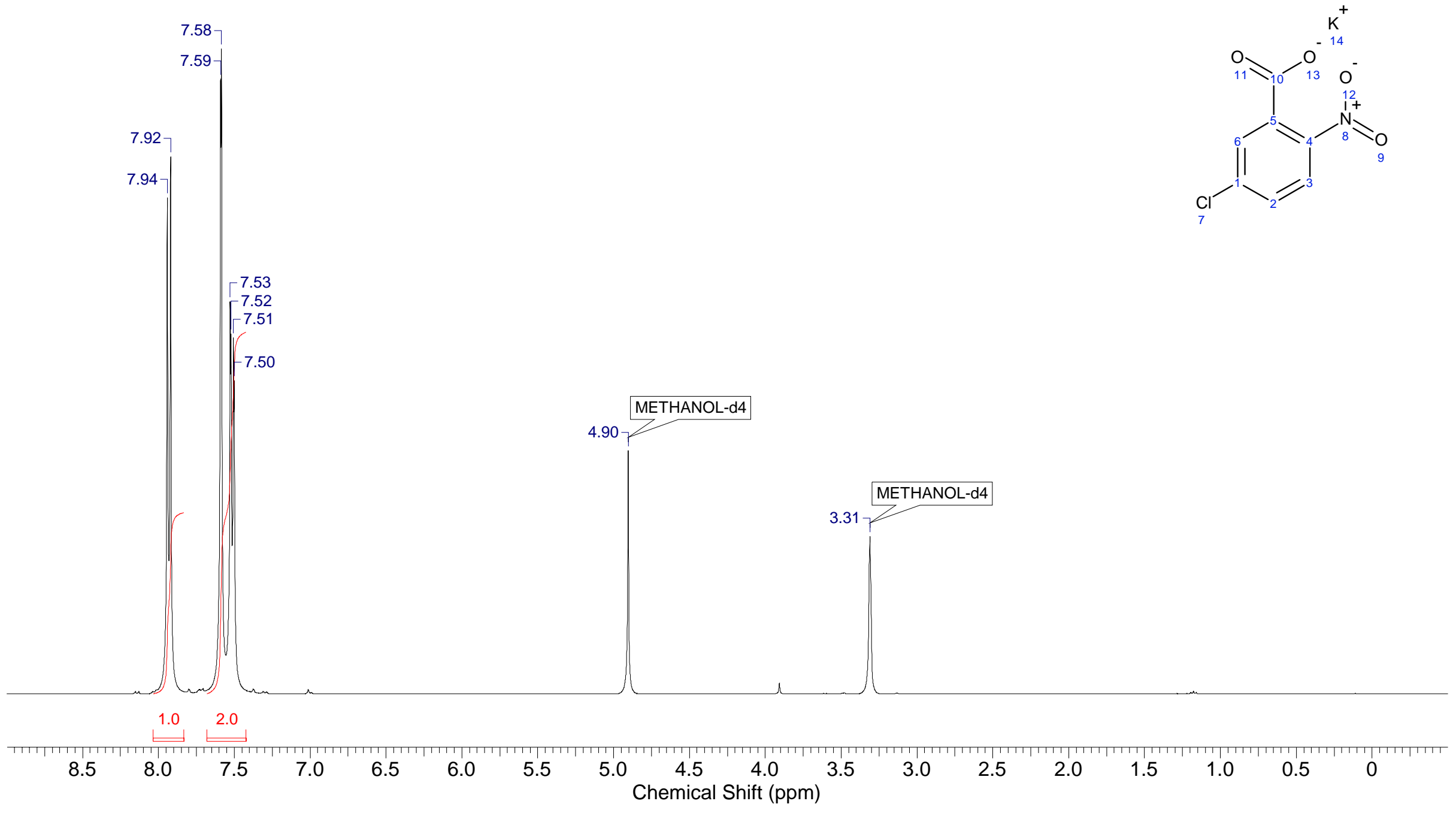


Potassium 5-chloro-2-nitrobenzoate (1e)

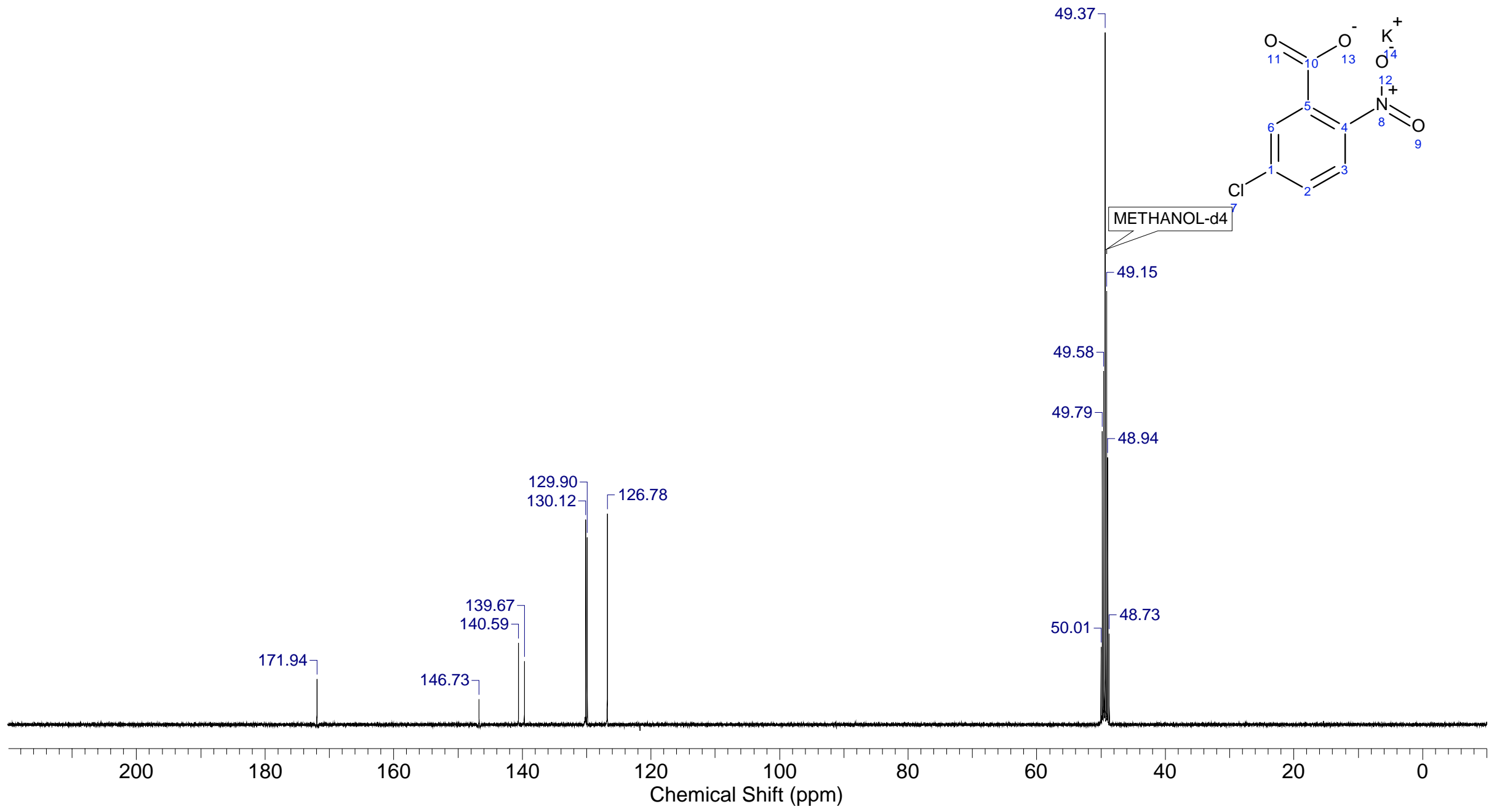


Potassium 5-methoxy-2-nitrobenzoate (1f)

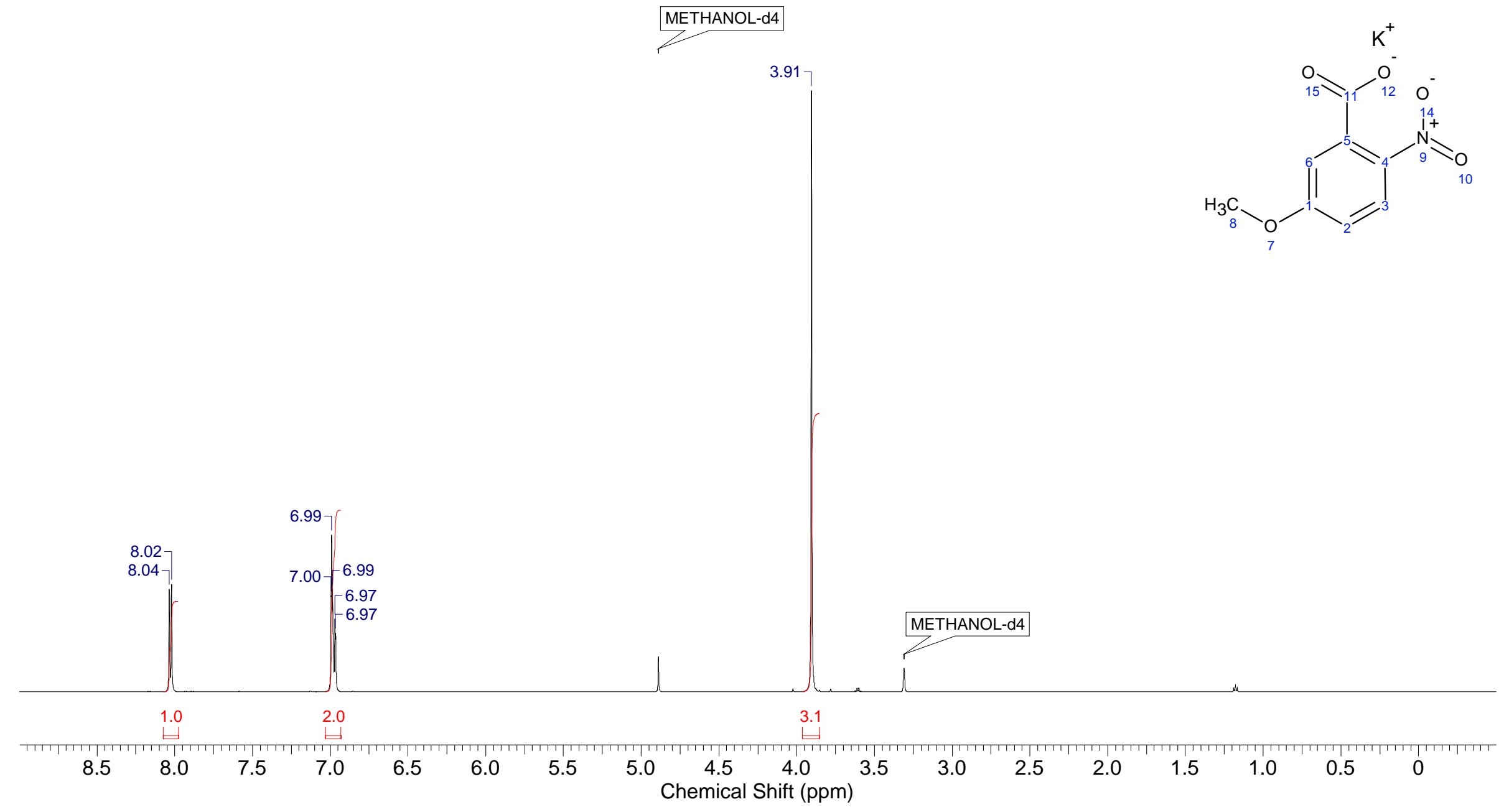


Potassium 5-methoxy-2-nitrobenzoate (1f)

${ }^{13} \mathrm{C}$ NMR, $101 \mathrm{MHz}, \mathrm{CD}_{3} \mathrm{OD}$

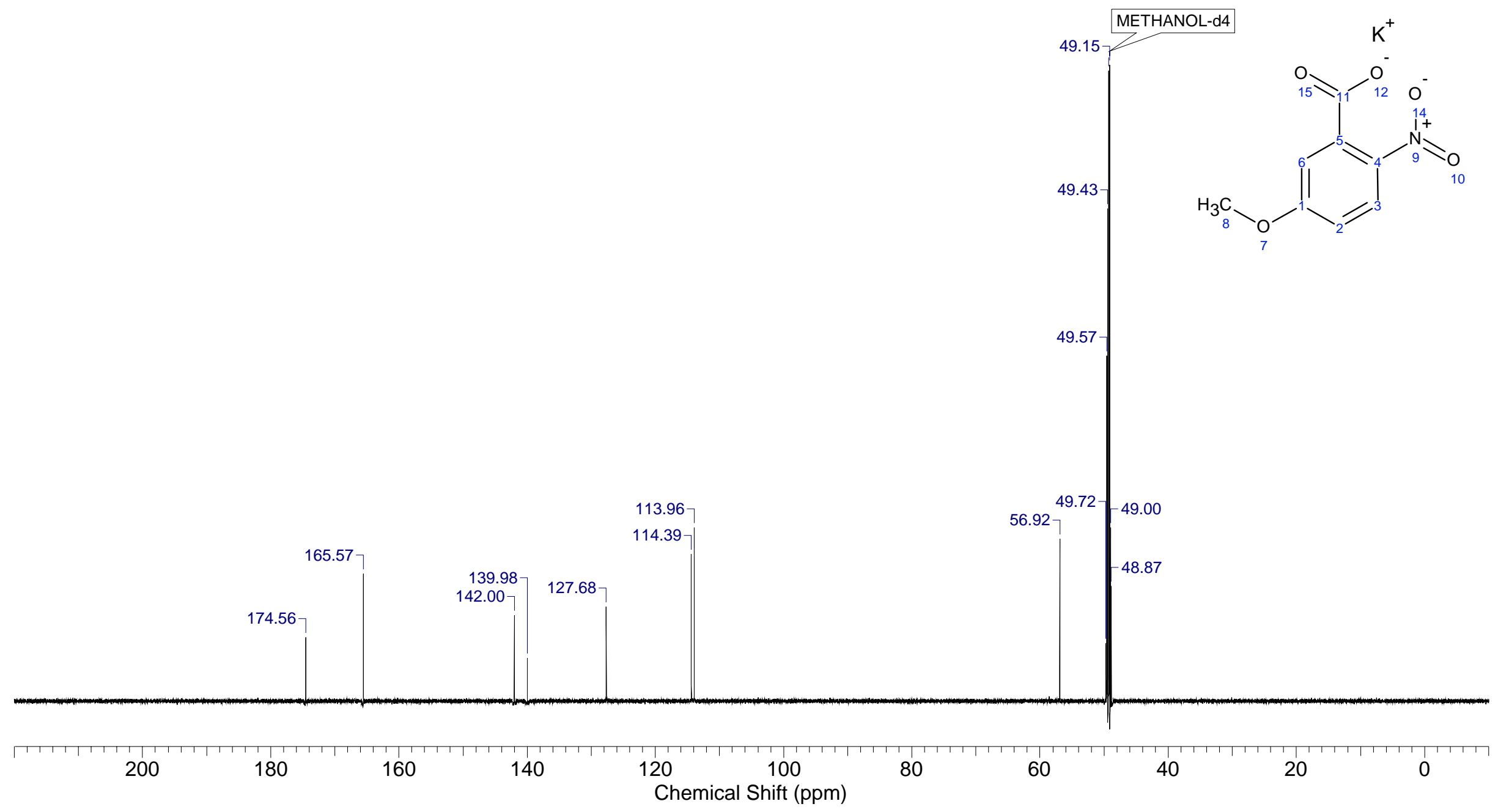


Potassium 2-methoxybenzoate (1g)

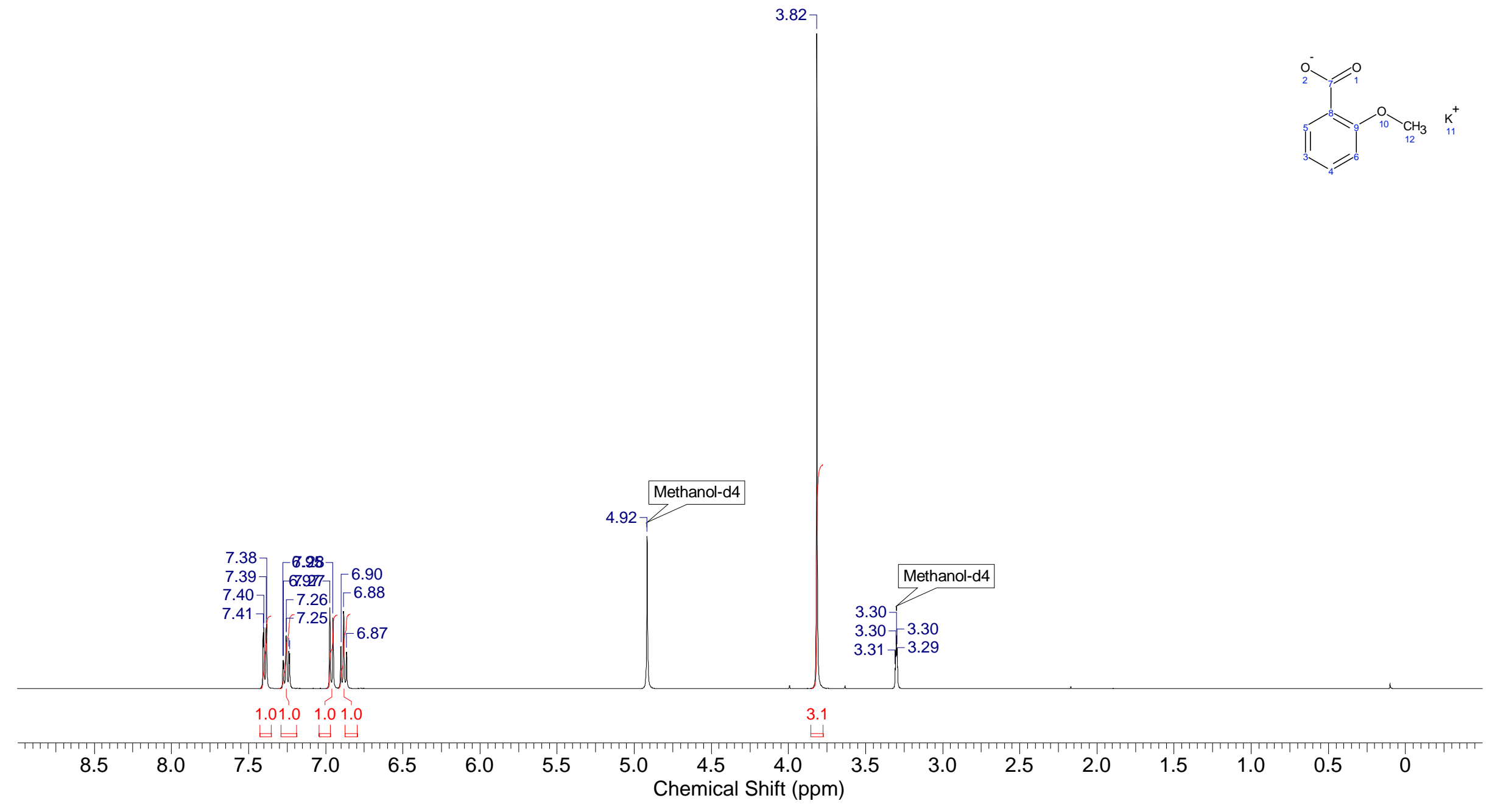


Potassium 2-methoxybenzoate (1g)

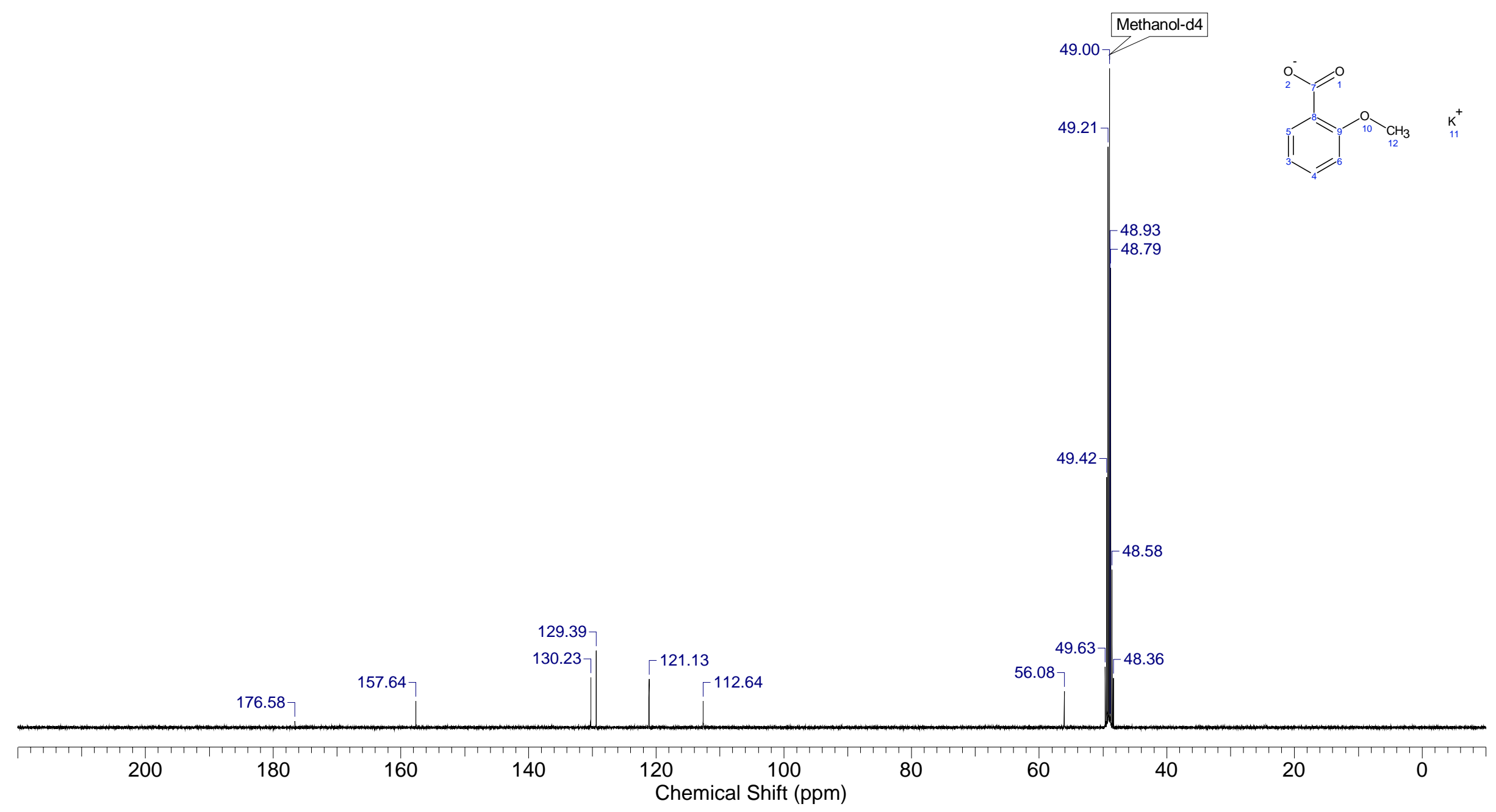


Potassium 2,6-dimethoxybenzoate (1h)

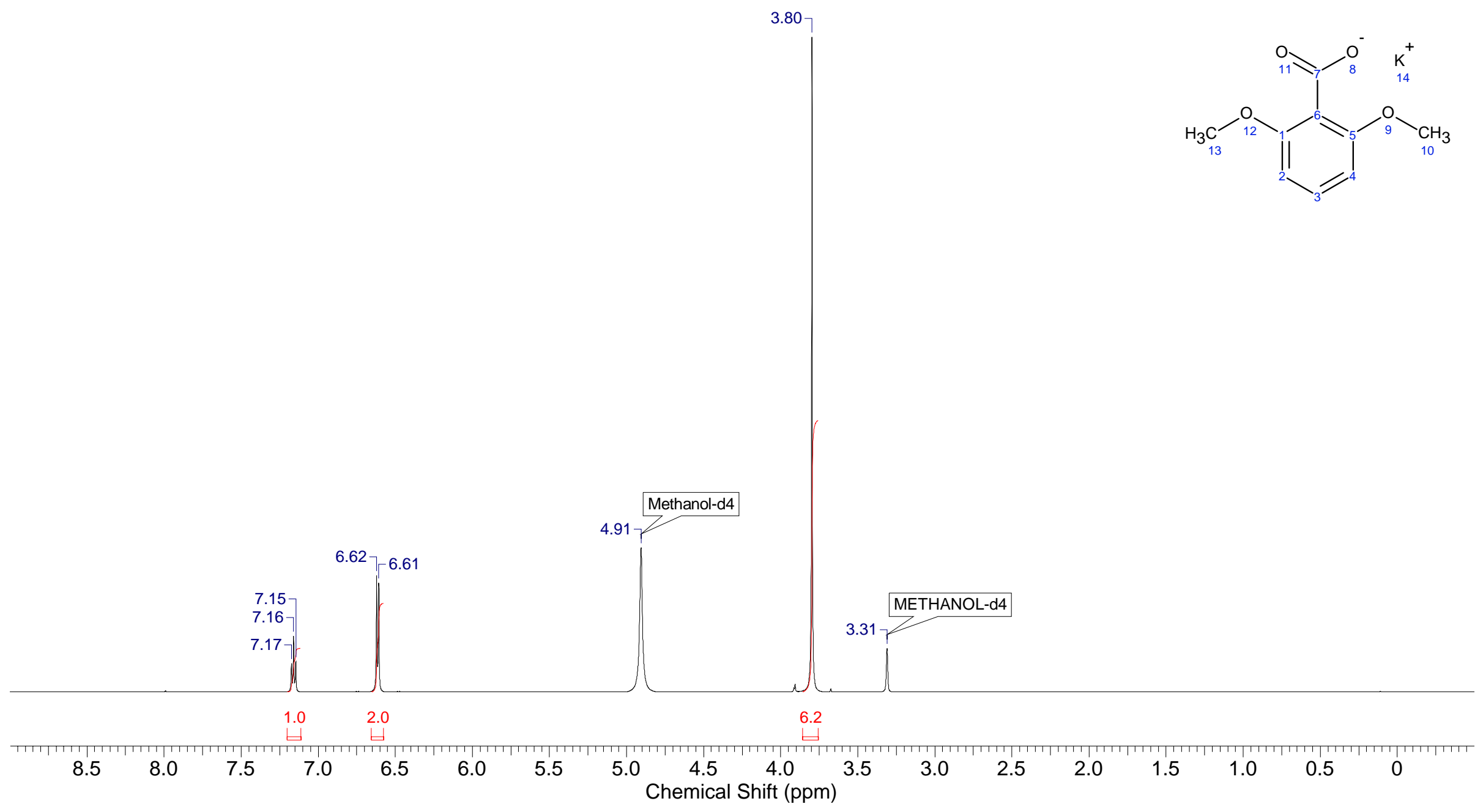


Potassium 2,6-dimethoxybenzoate (1h)

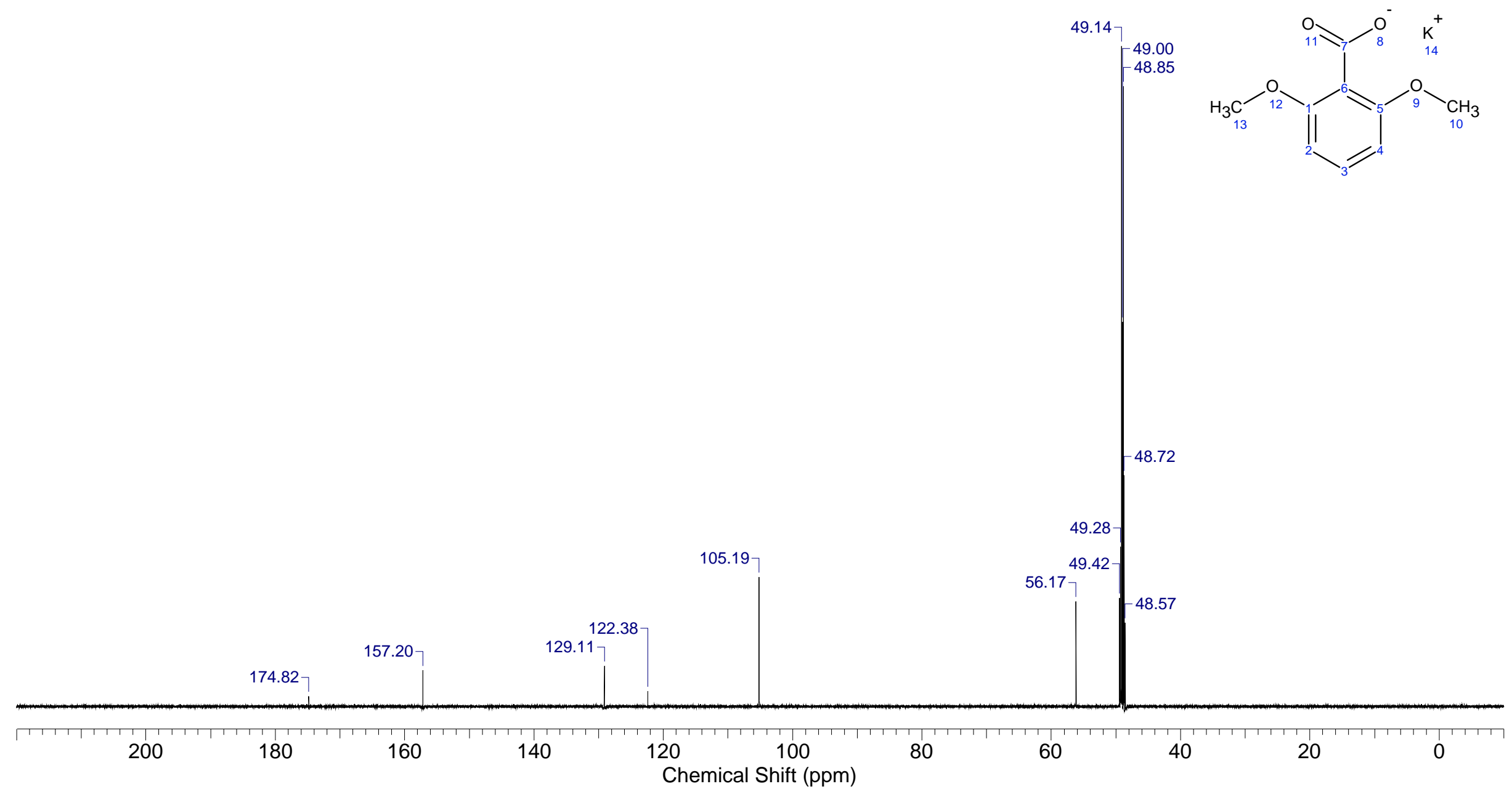


Potassium 2-(methylsulfonyl)benzoate (1i)

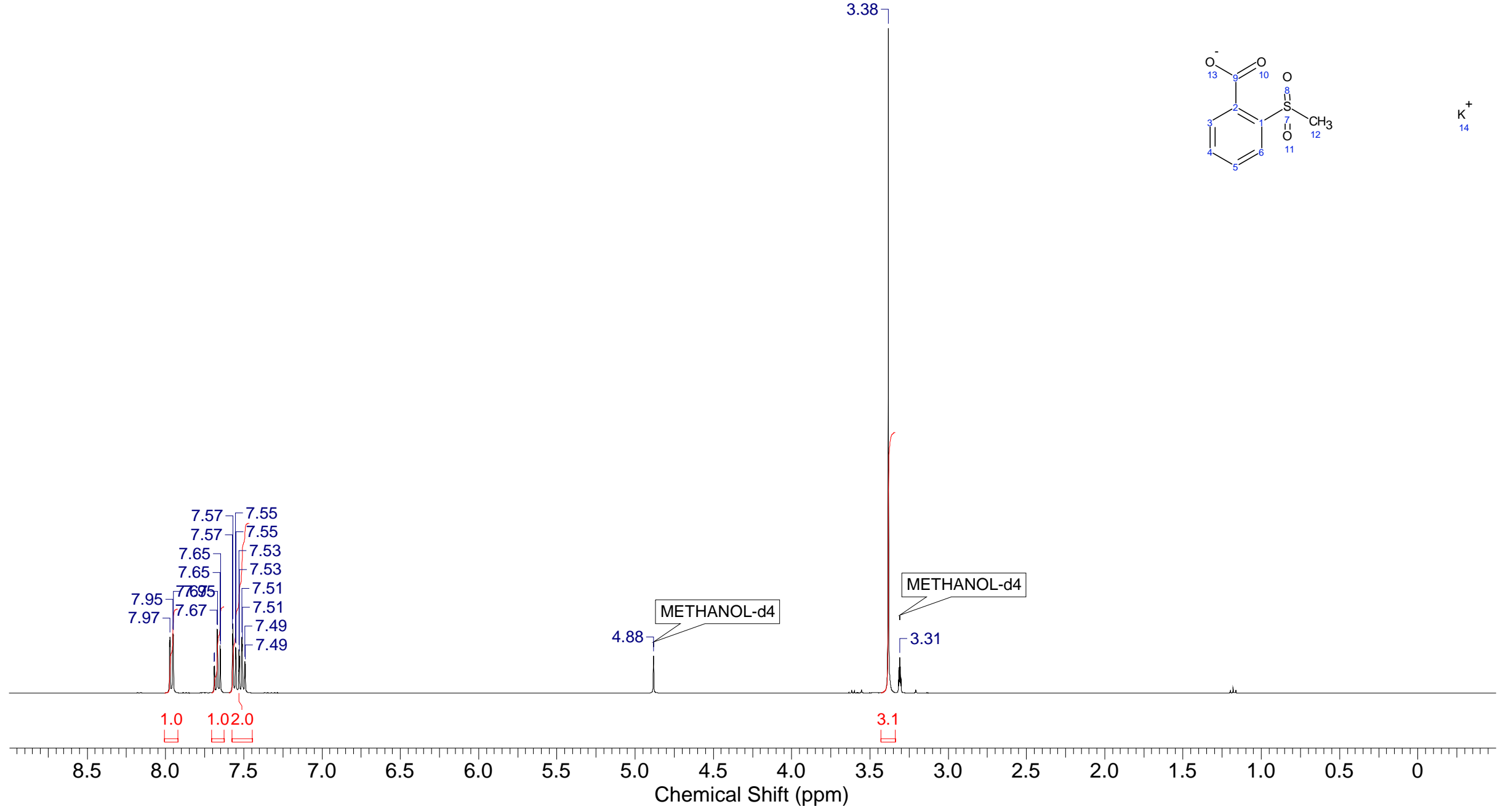


Potassium 2-(methylsulfonyl)benzoate (1i)

${ }^{13} \mathrm{C}$ NMR, $101 \mathrm{MHz}, \mathrm{CD}_{3} \mathrm{OD}$

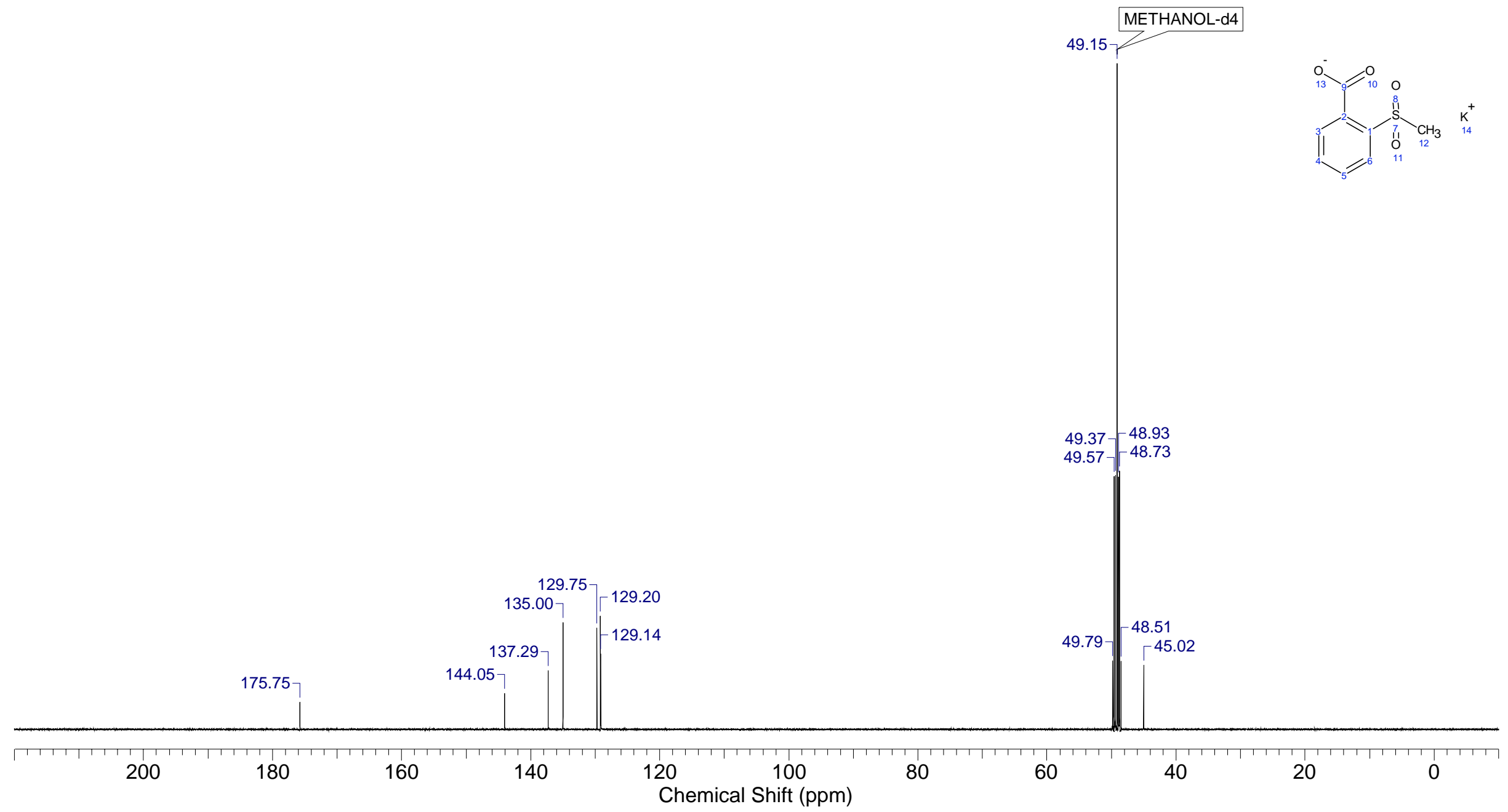


Potassium 2-(dimethylamino)benzoate (1j)

${ }^{1} \mathrm{H}$ NMR, $400 \mathrm{MHz}, \mathrm{CD}_{3} \mathrm{OD}$

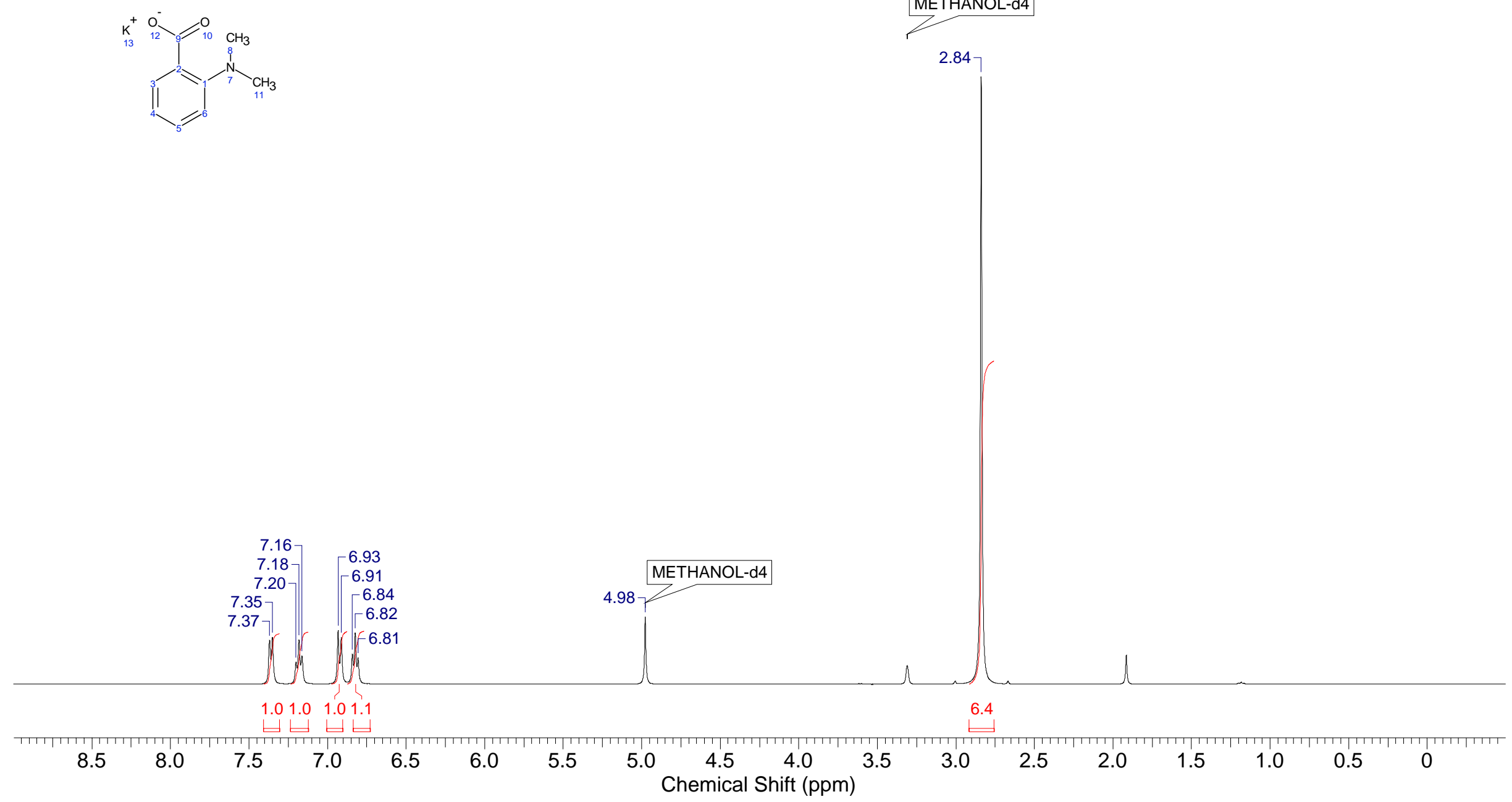

METHANOL-d4 
Potassium 2-(dimethylamino)benzoate (1j)

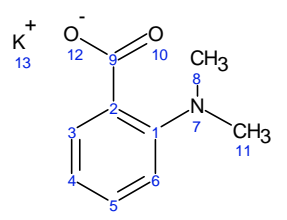

${ }^{13} \mathrm{C}$ NMR, $101 \mathrm{MHz}, \mathrm{CD}_{3} \mathrm{OD}$

METHANOL-d4

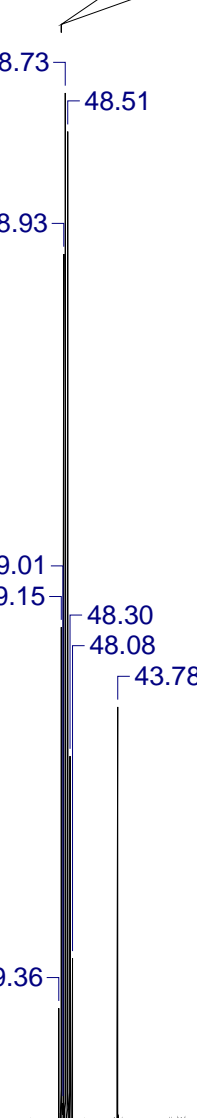

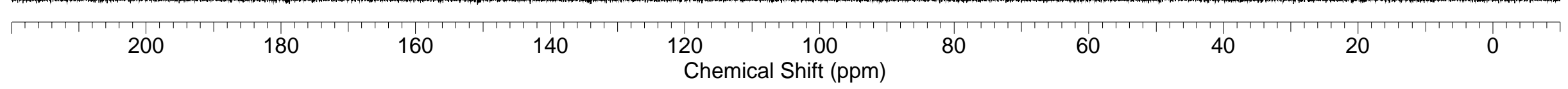


Potassium 2-chloro-5-nitrobenzoate (1k)

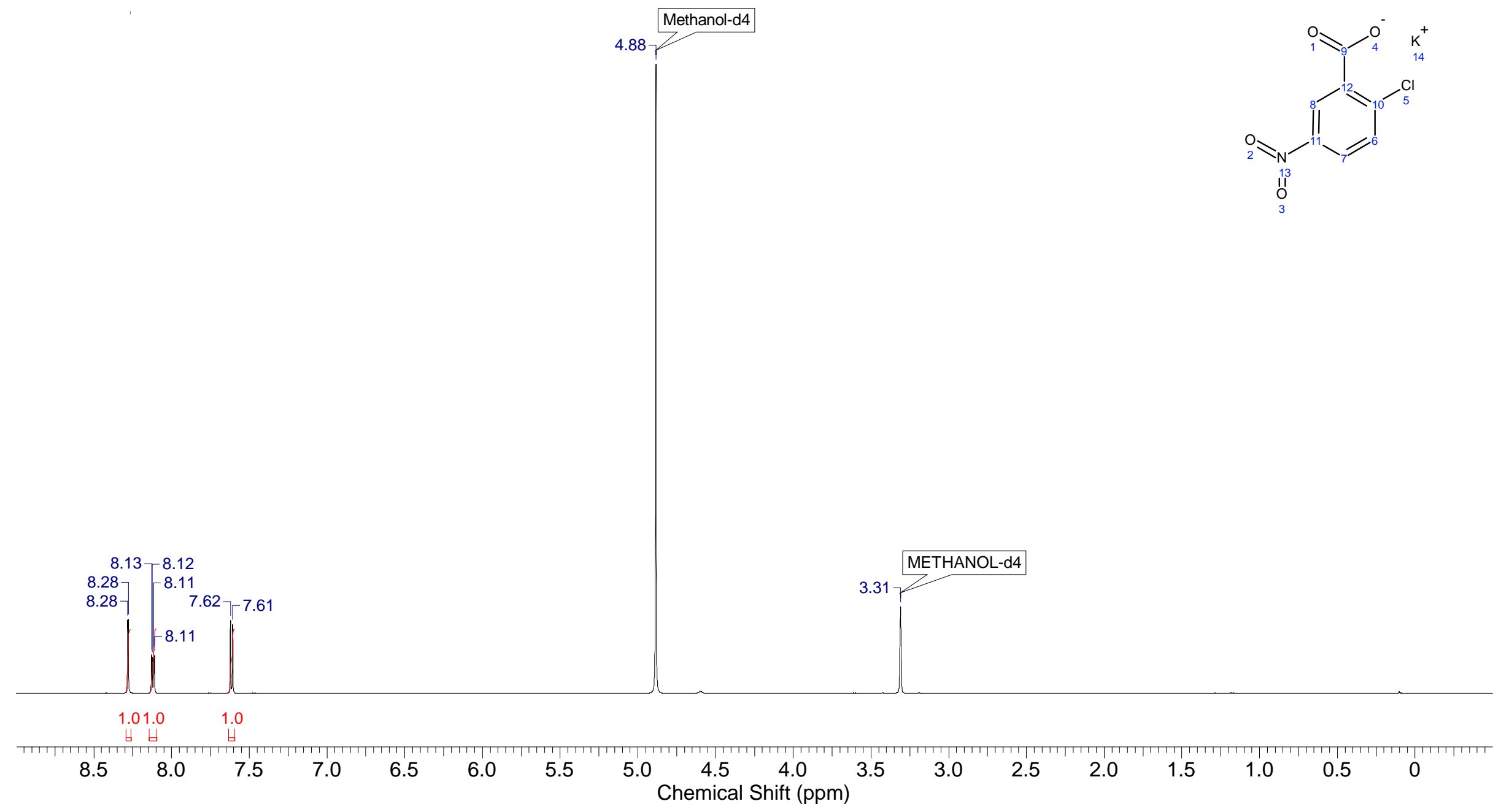


Potassium 2-chloro-5-nitrobenzoate (1k)

${ }^{13} \mathrm{C}$ NMR, $101 \mathrm{MHz}, \mathrm{CD}_{3} \mathrm{OD}$

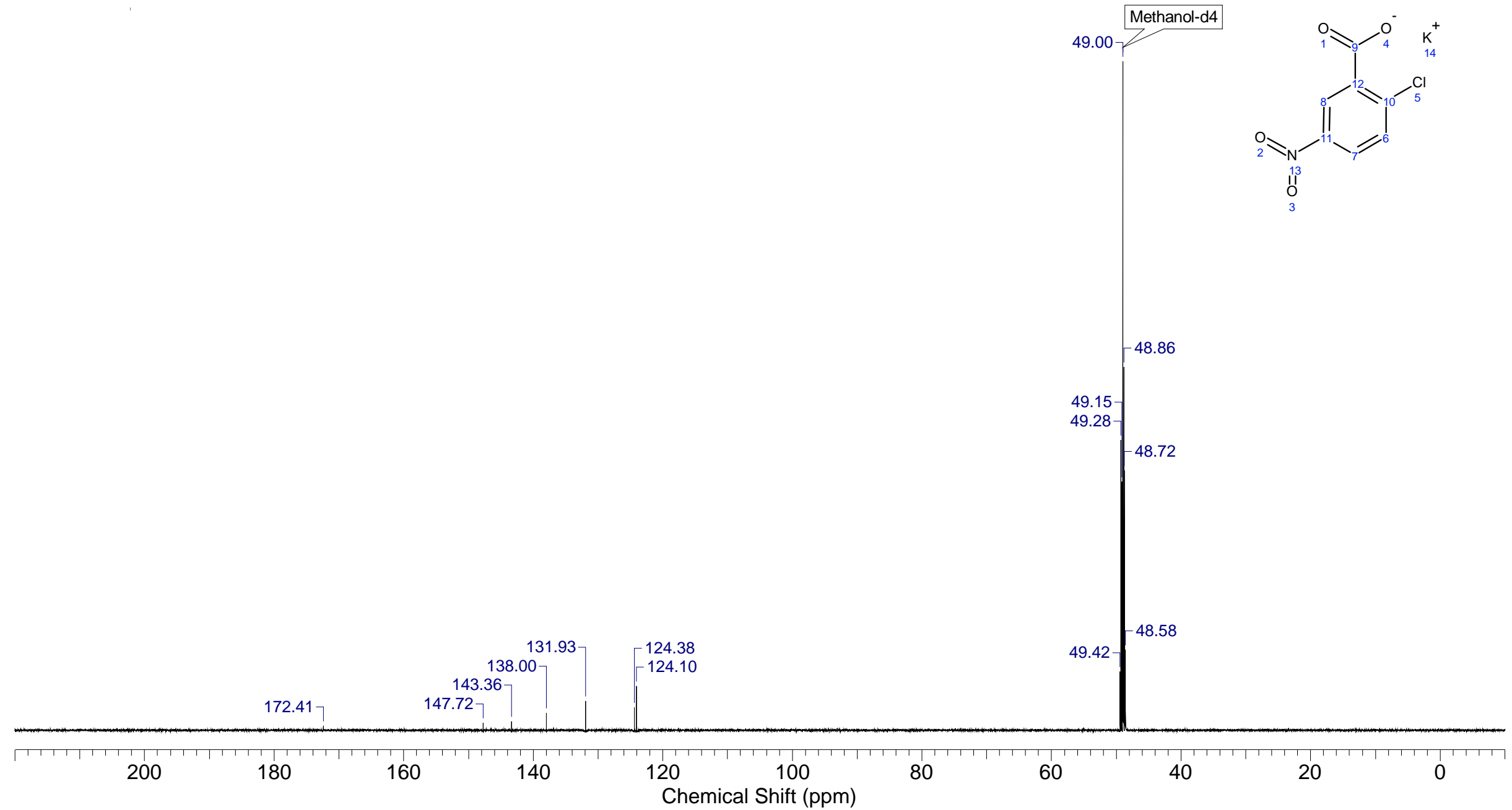


Potassium 2-fluorobenzoate (11)

${ }^{1} \mathrm{H}$ NMR, $400 \mathrm{MHz}, \mathrm{D}_{2} \mathrm{O}$

DEUTERIUM OXIDE
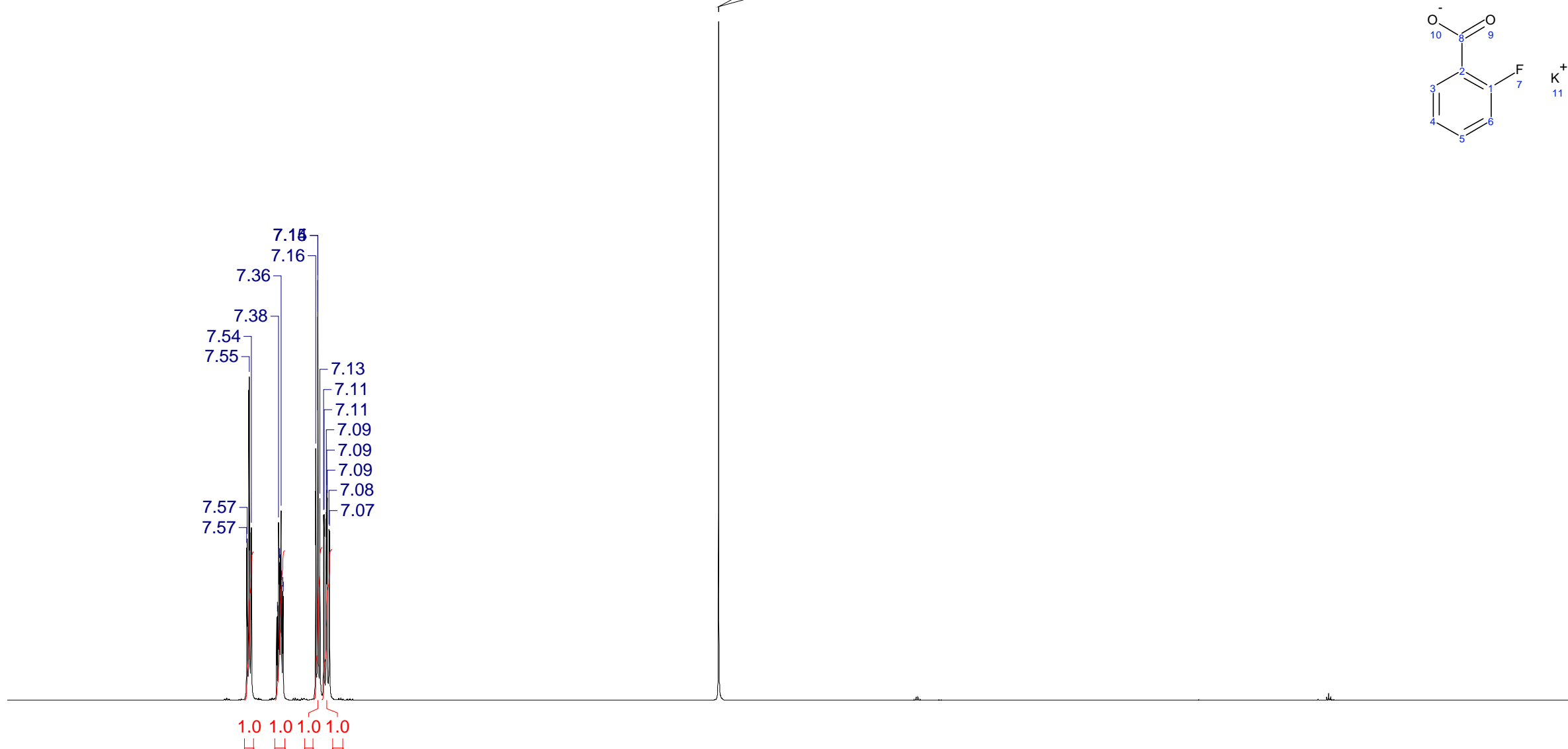

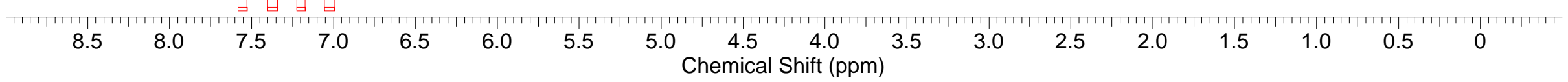


Potassium 2-fluorobenzoate (11)

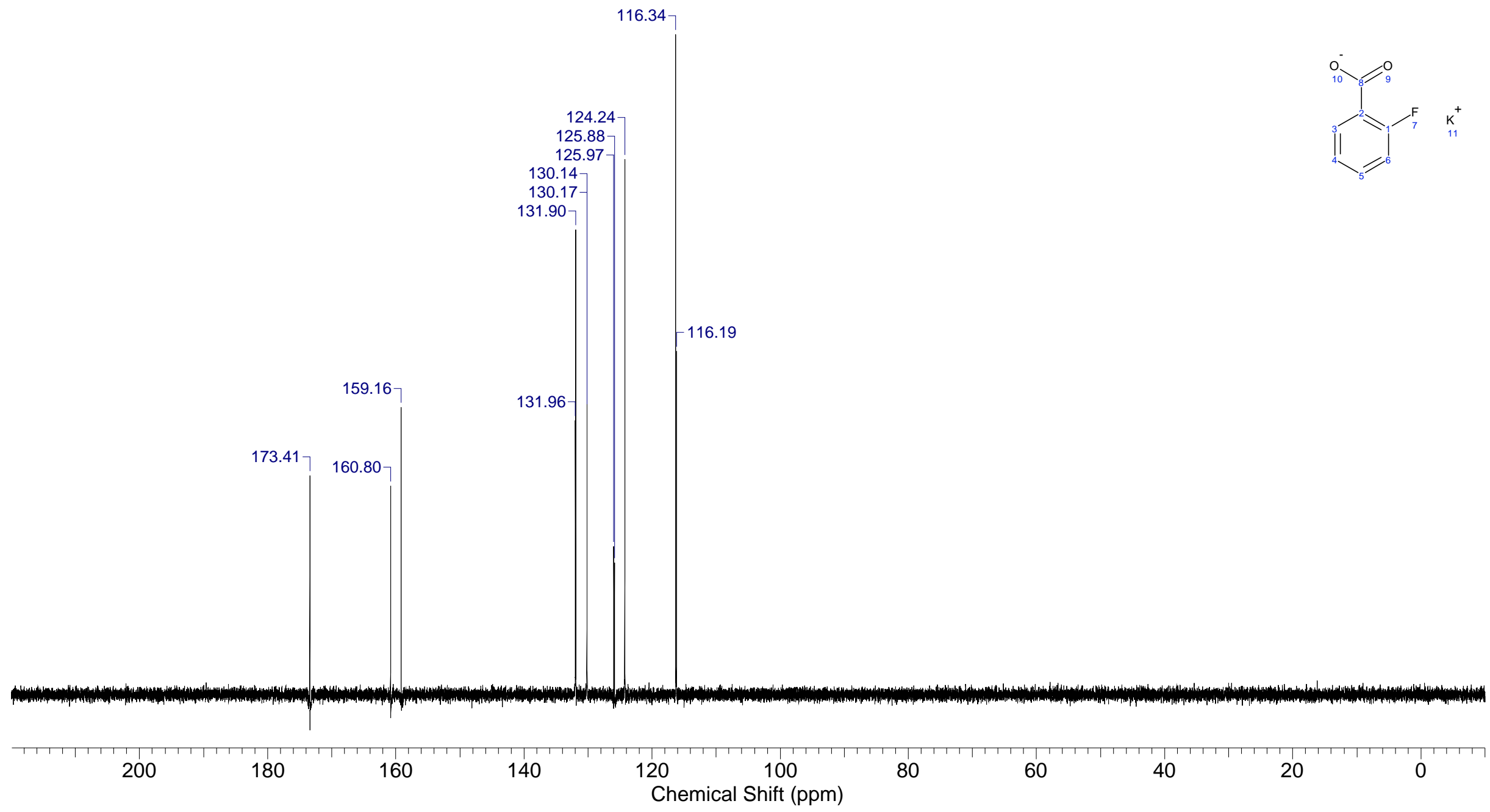


Potassium 2-trifluoromethylbenzoate (1m)

${ }^{1} \mathrm{H}$ NMR, $400 \mathrm{MHz}, \mathrm{CD}_{3} \mathrm{OD}$

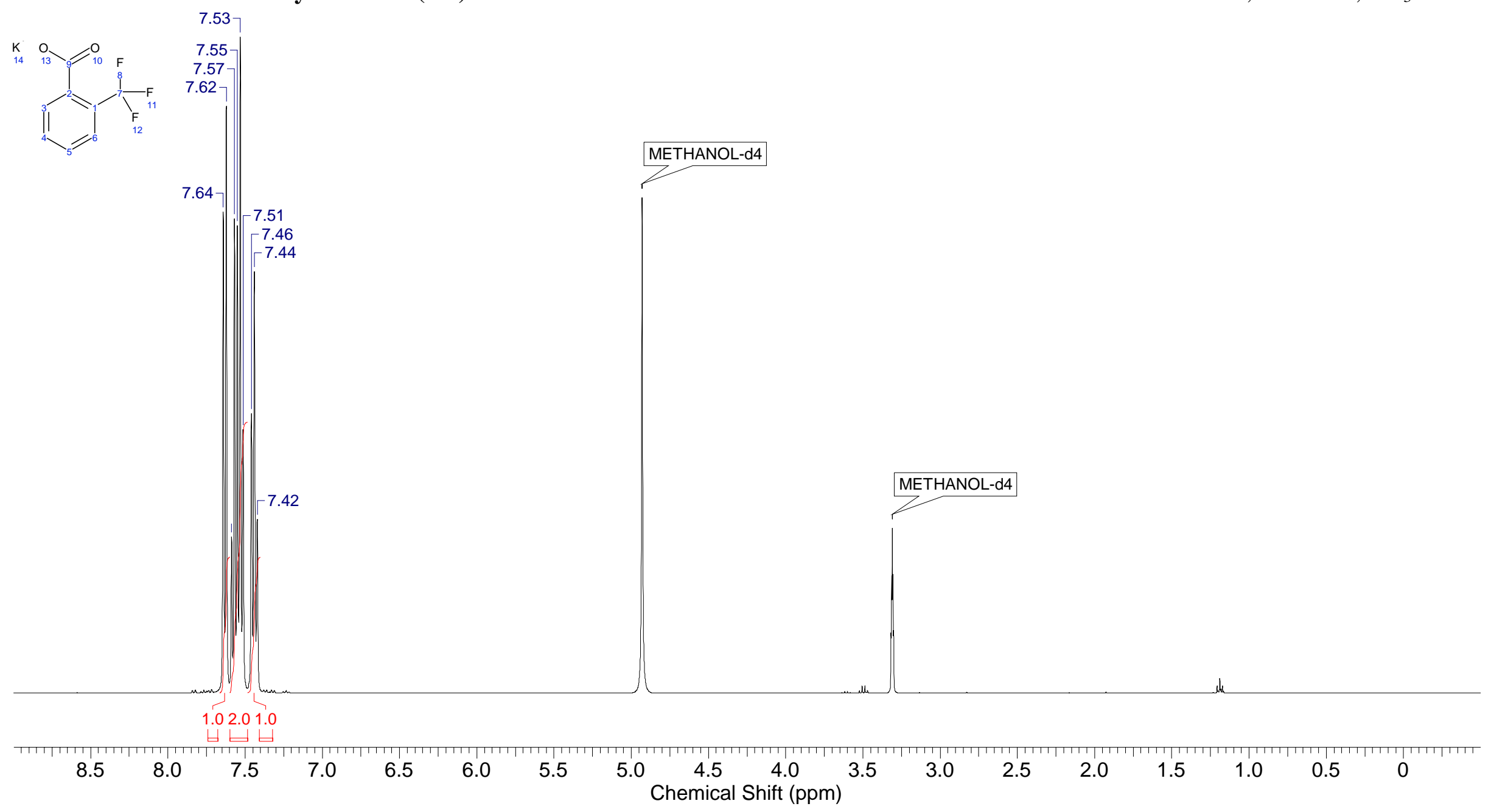


Potassium 2-trifluoromethylbenzoate (1m)

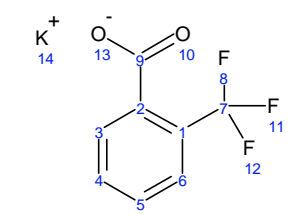

${ }^{13} \mathrm{C}$ NMR, $101 \mathrm{MHz}, \mathrm{CD}_{3} \mathrm{OD}$

METHANOL-d4

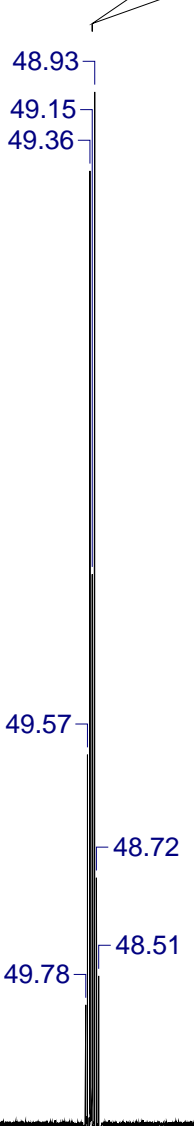

60
40
20

Chemical Shift (ppm) 
Potassium 2-benzoylbenzoate (1n)

${ }^{1} \mathrm{H}$ NMR, $400 \mathrm{MHz}, \mathrm{D}_{2} \mathrm{O}$

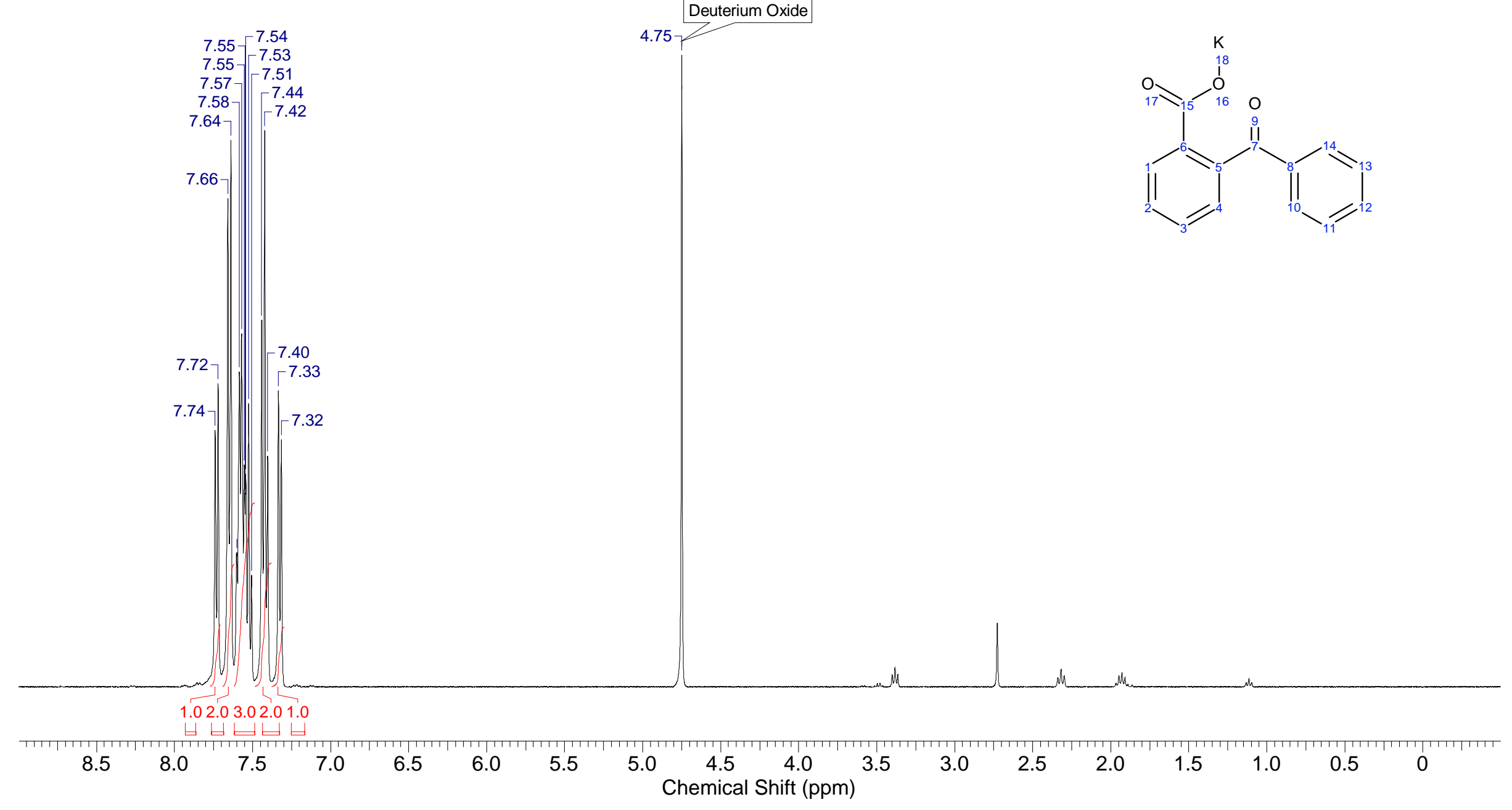


Potassium 2-benzoylbenzoate (1n)

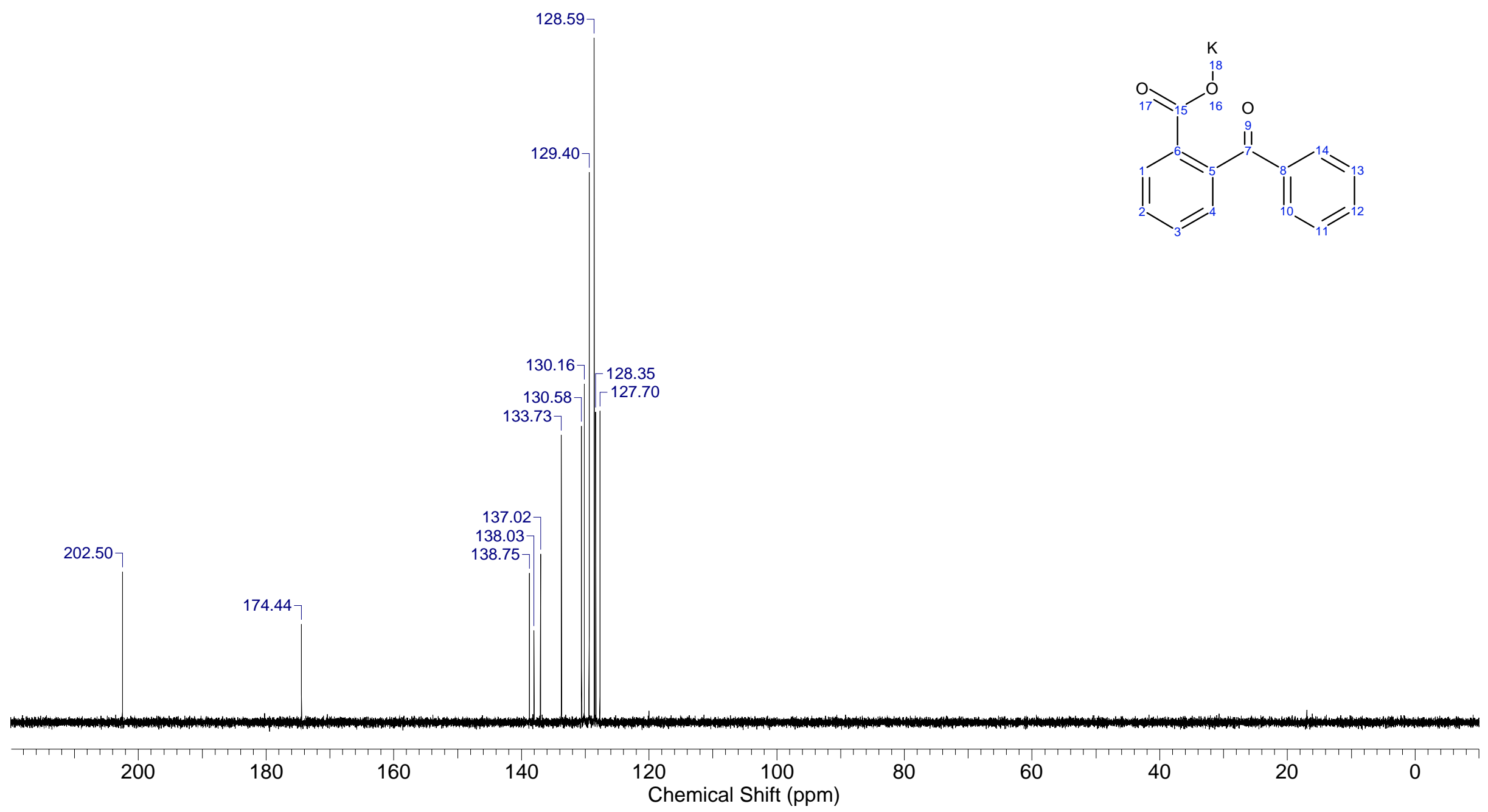


Potassium (1,1'-biphenyl)-2-carboxylate (10)

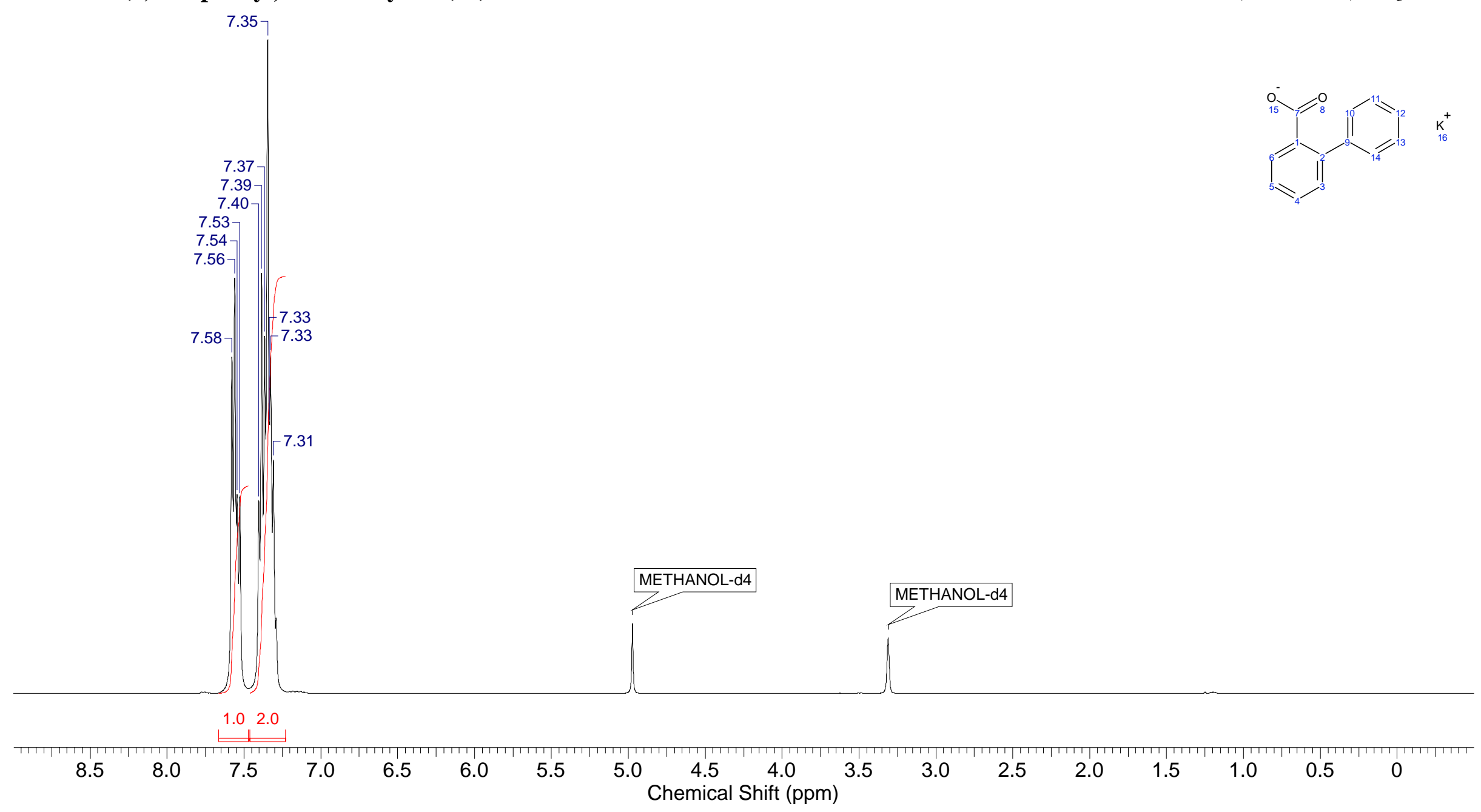


Potassium (1,1'-biphenyl)-2-carboxylate (10)

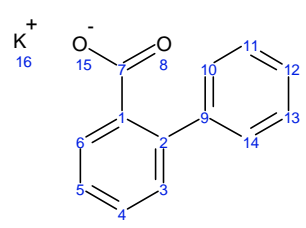

${ }^{13} \mathrm{C}$ NMR, $101 \mathrm{MHz}, \mathrm{CD}_{3} \mathrm{OD}$

49.37 METHANOL-d4

49.15

49.57

49.79

$-48.94$

$-48.73$

50.00

179.28

$143.67\urcorner$

$\left[\begin{array}{r}128.51 \\ -128.17\end{array}\right.$

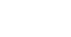

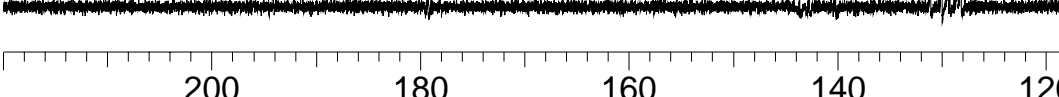

120

100

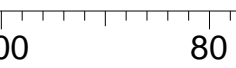

80

20

Chemical Shift (ppm) 
1-Methoxy-2-nitrobenzene (3aa)

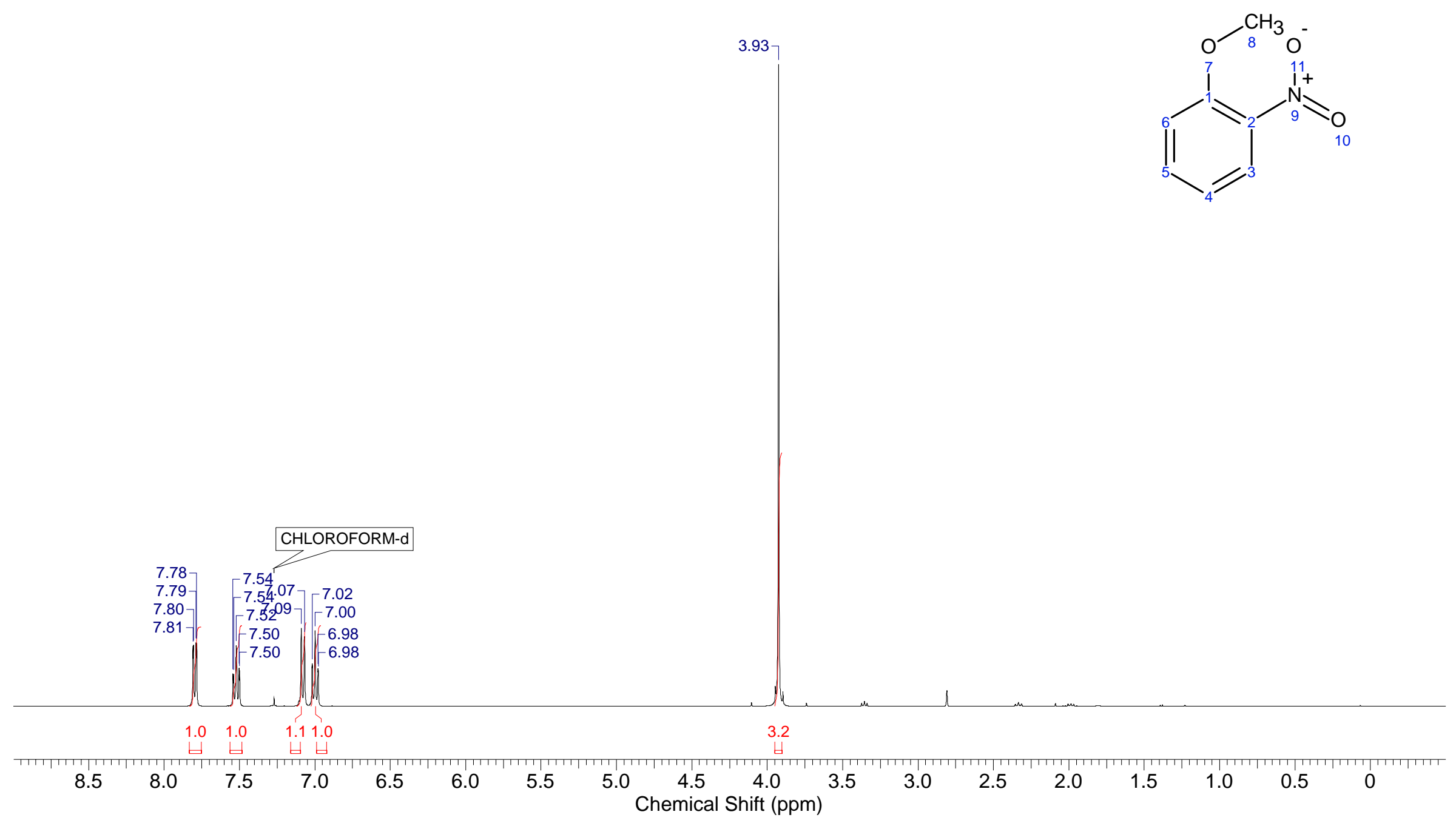




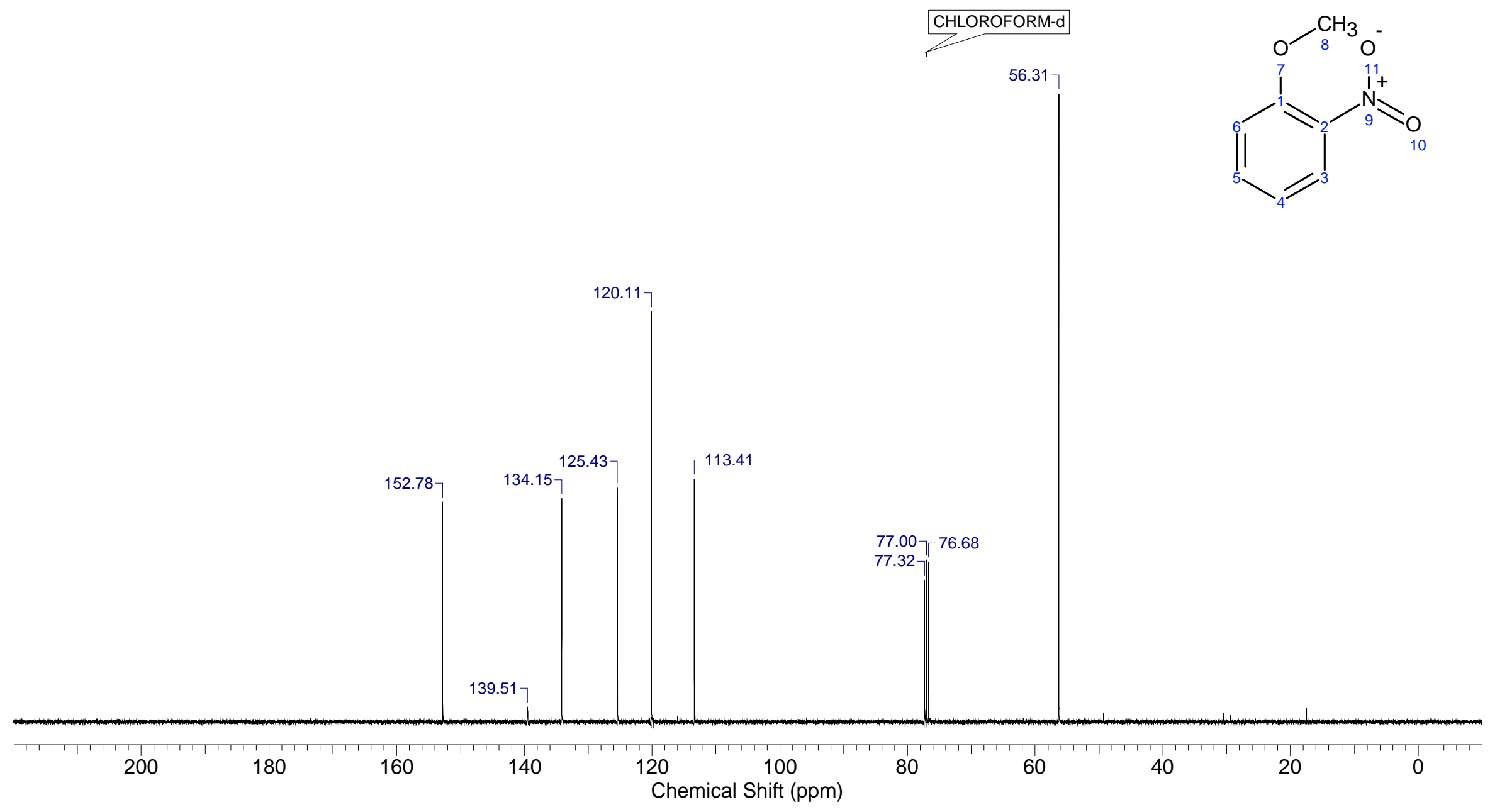




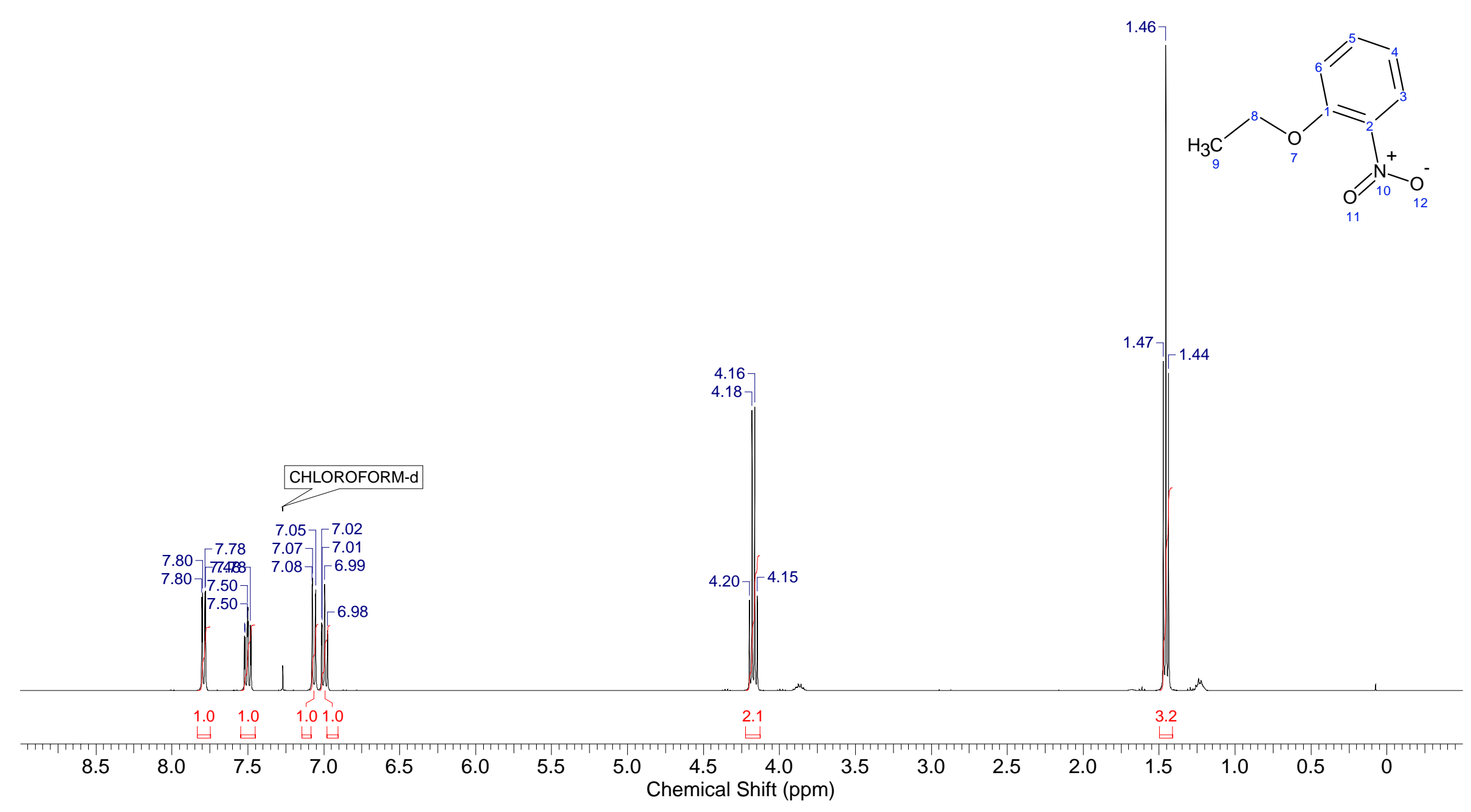


1-Ethoxy-2-nitrobenzene (3ab)

${ }^{13} \mathrm{C}$ NMR, $101 \mathrm{MHz}, \mathrm{CDCl}_{3}$
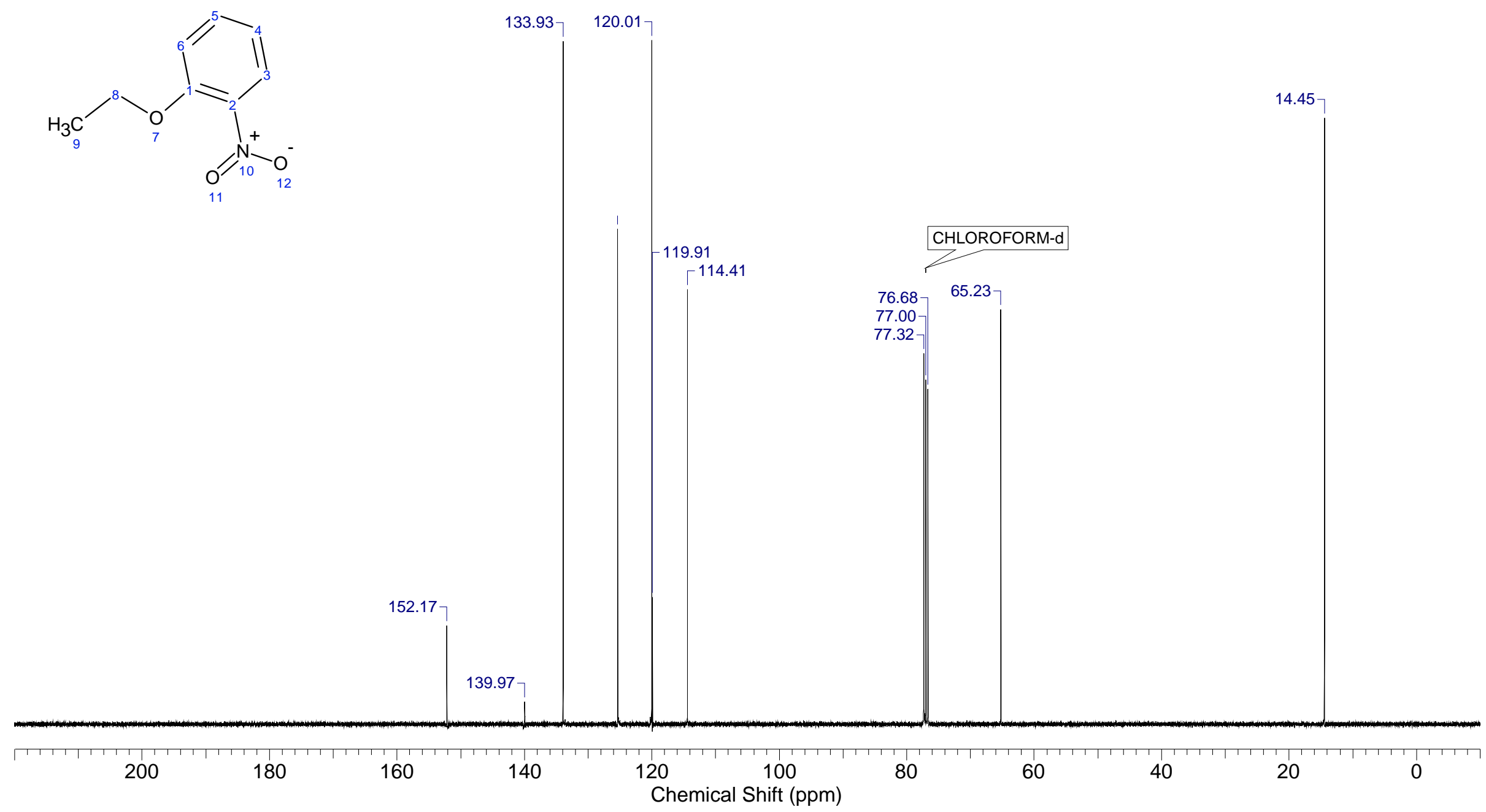

$14.45-$ 

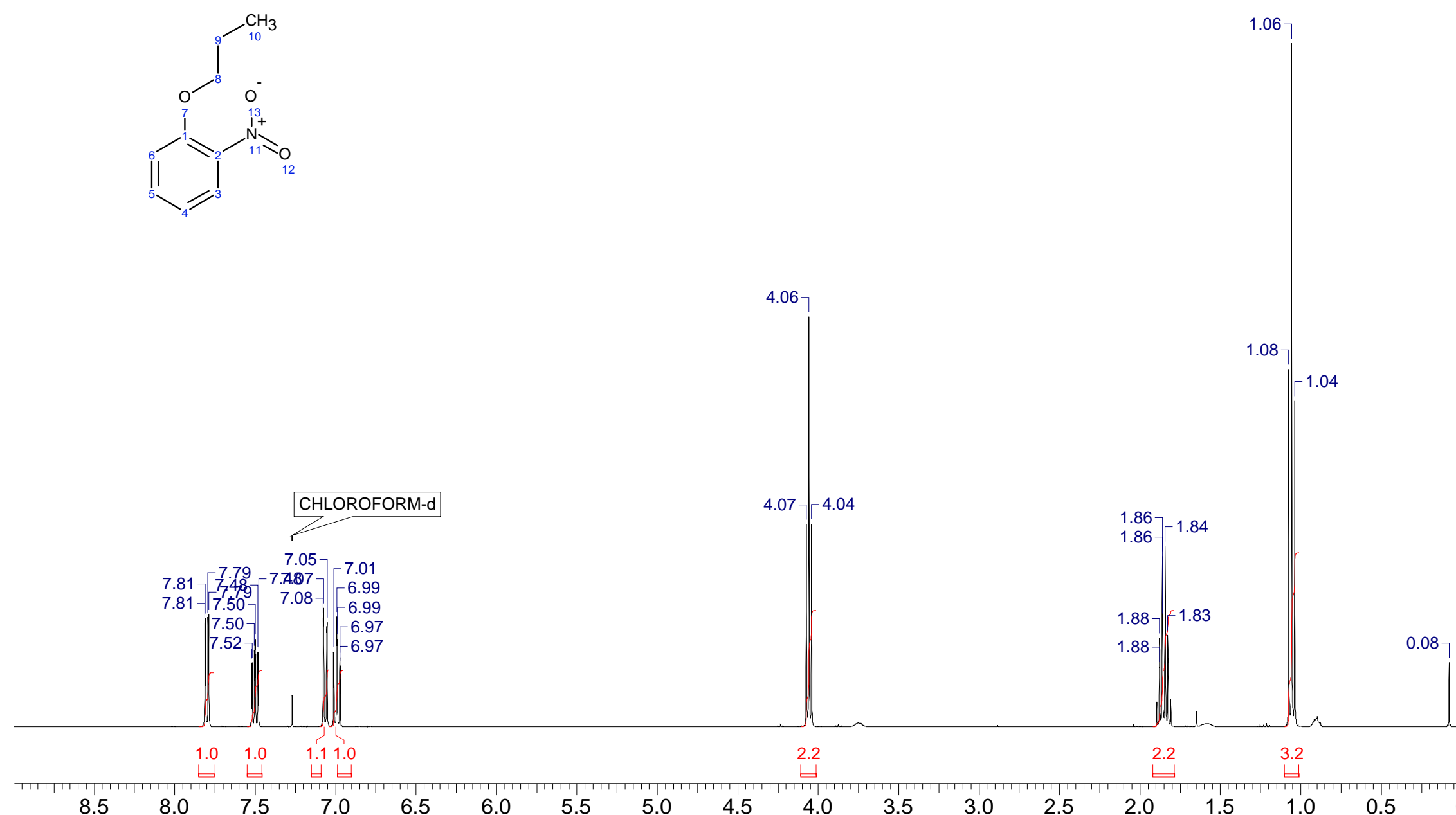

$6.0 \quad 5.5$

$5.0 \quad 4.5$

Chemical Shift (ppm) 


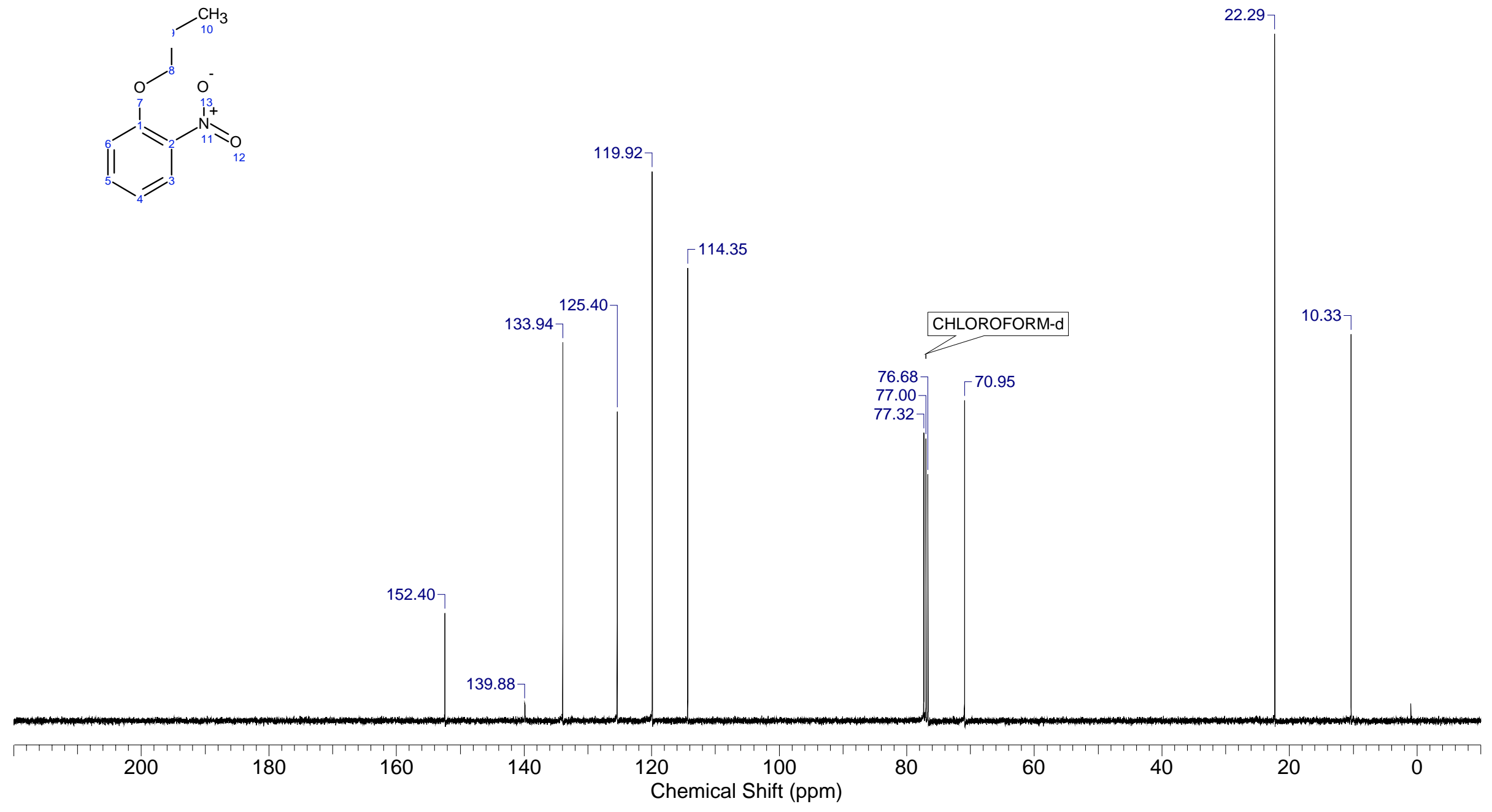



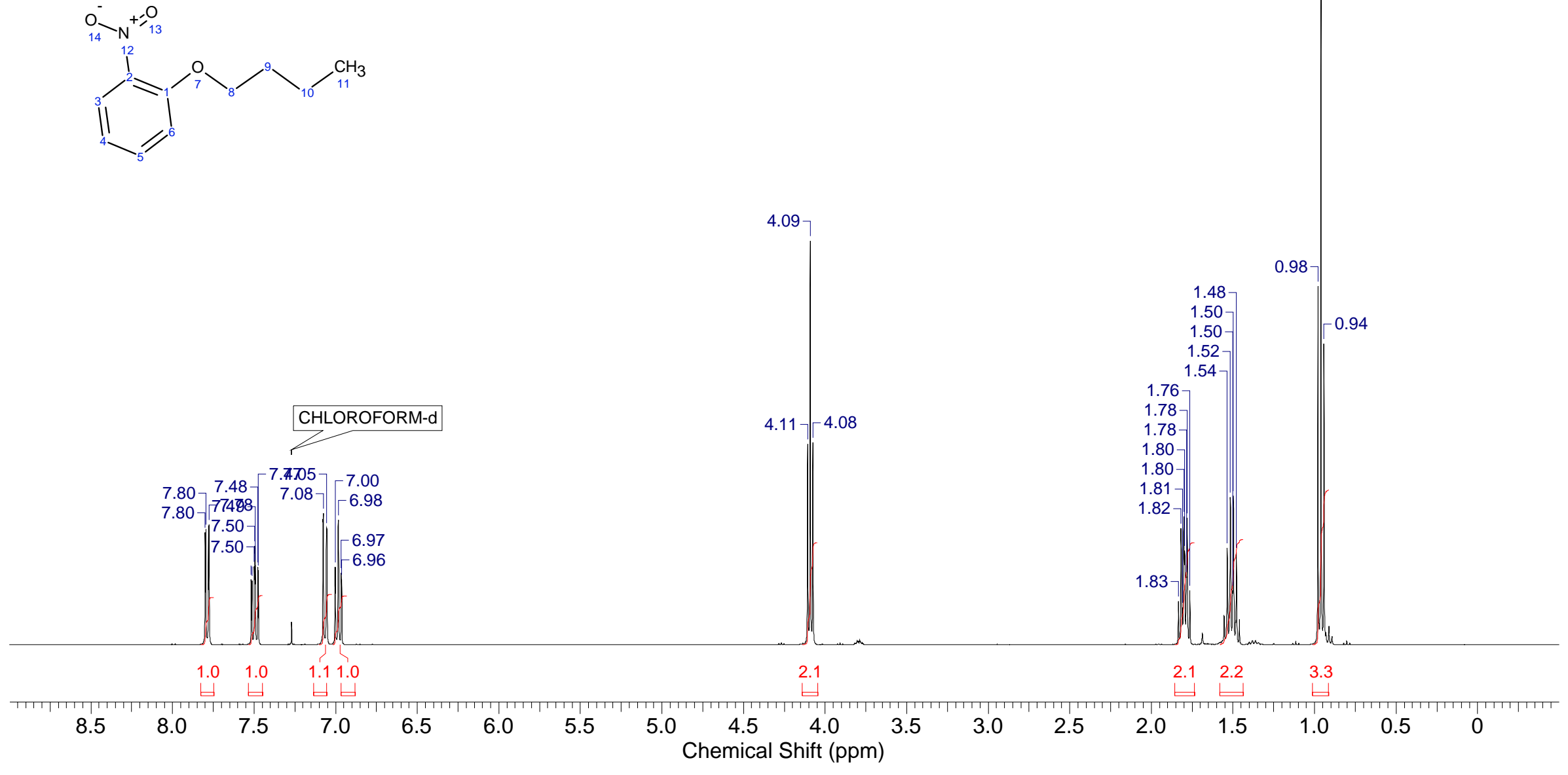
1-Butoxy-2-nitrobenzene (3ad)

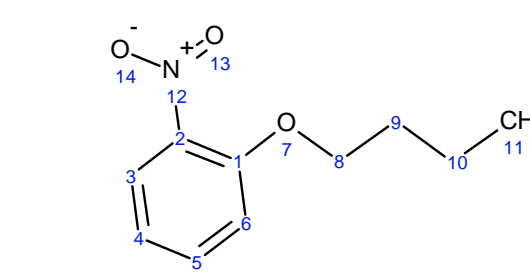

${ }^{13} \mathrm{C}$ NMR, $101 \mathrm{MHz}, \mathrm{CDCl}_{3}$

8.98

69.17

$\Gamma^{114.30}$

$77.00-$
$77.32-$

77.32

152.36

139.83
30.87

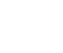



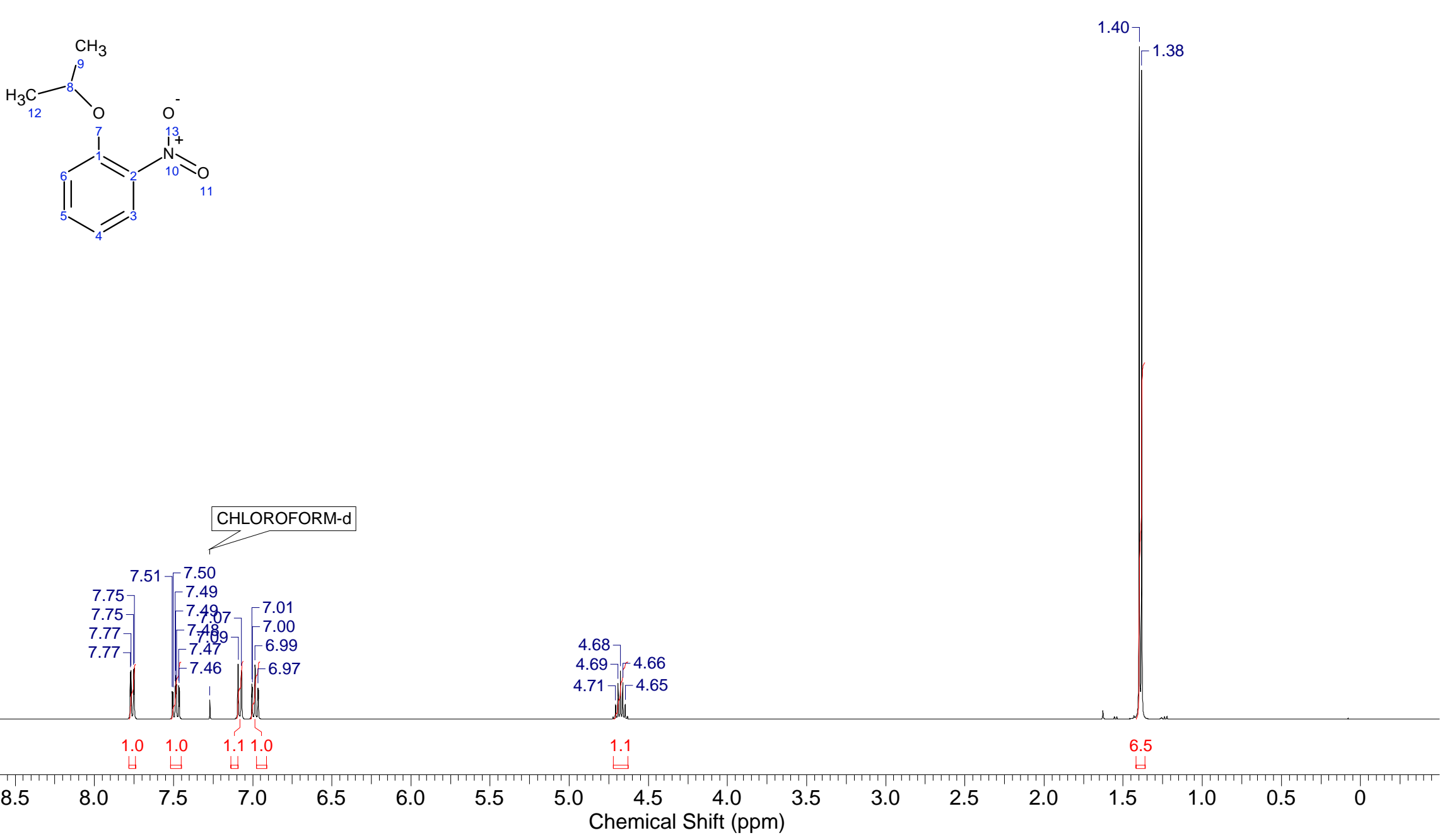

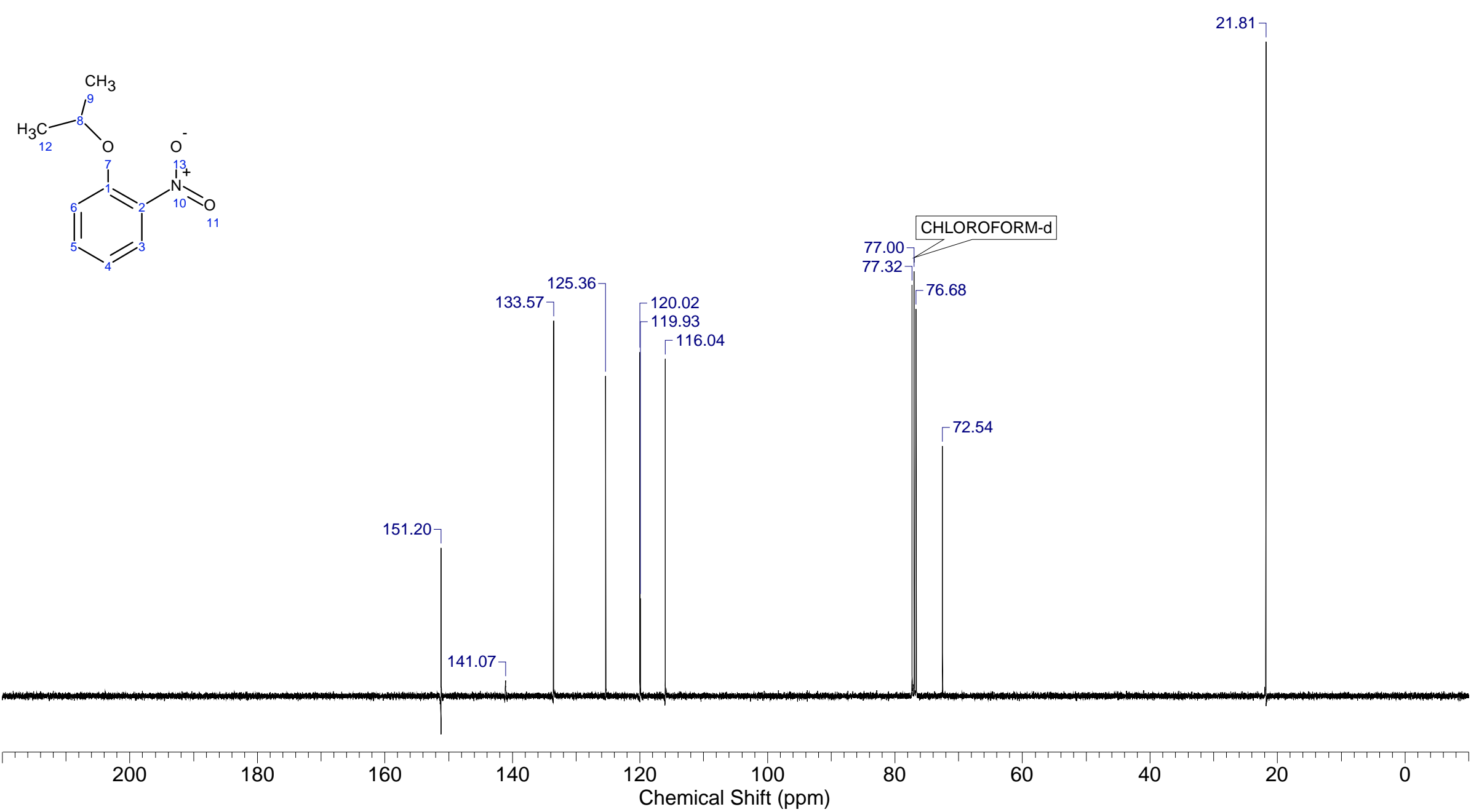


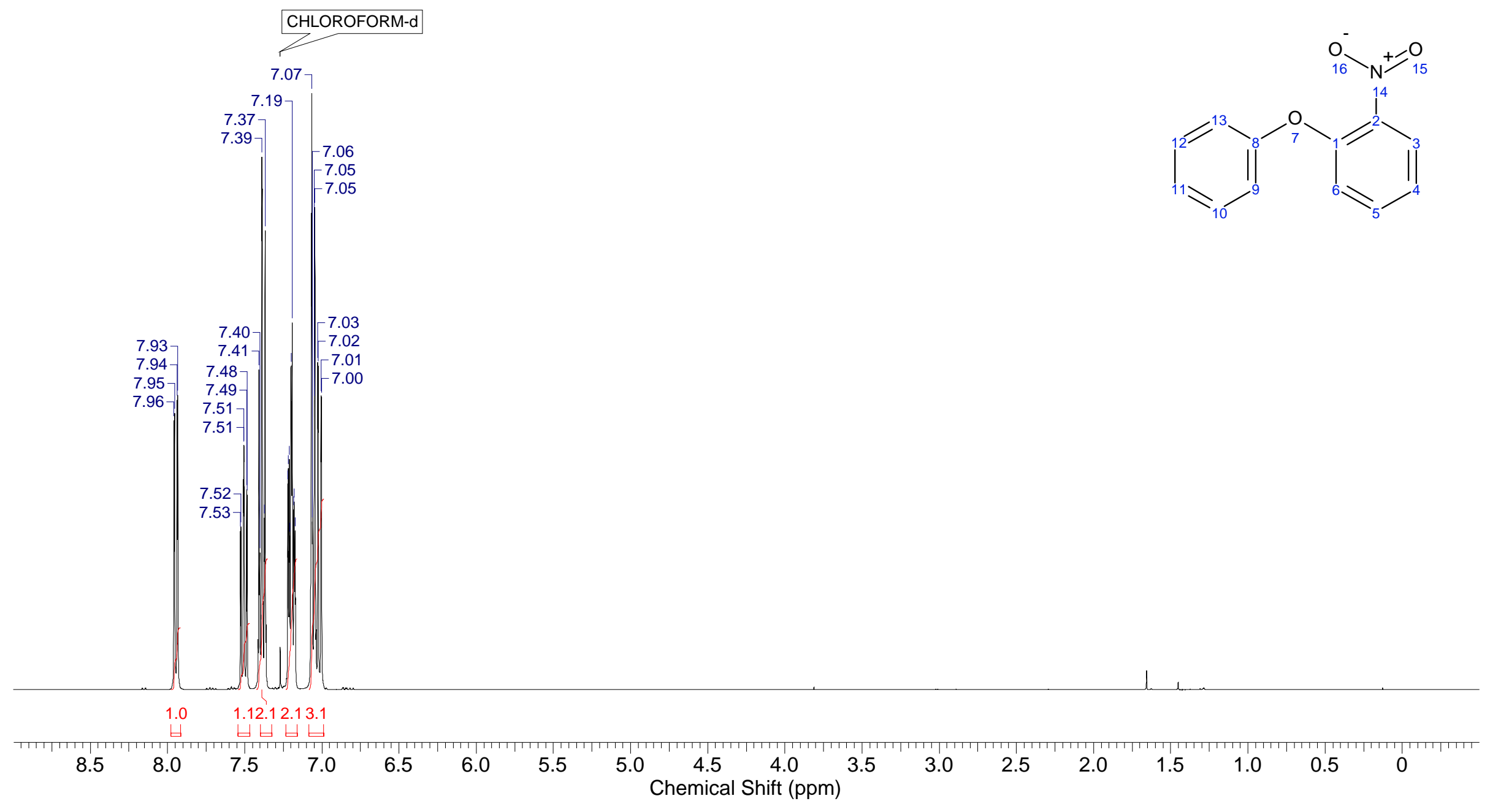




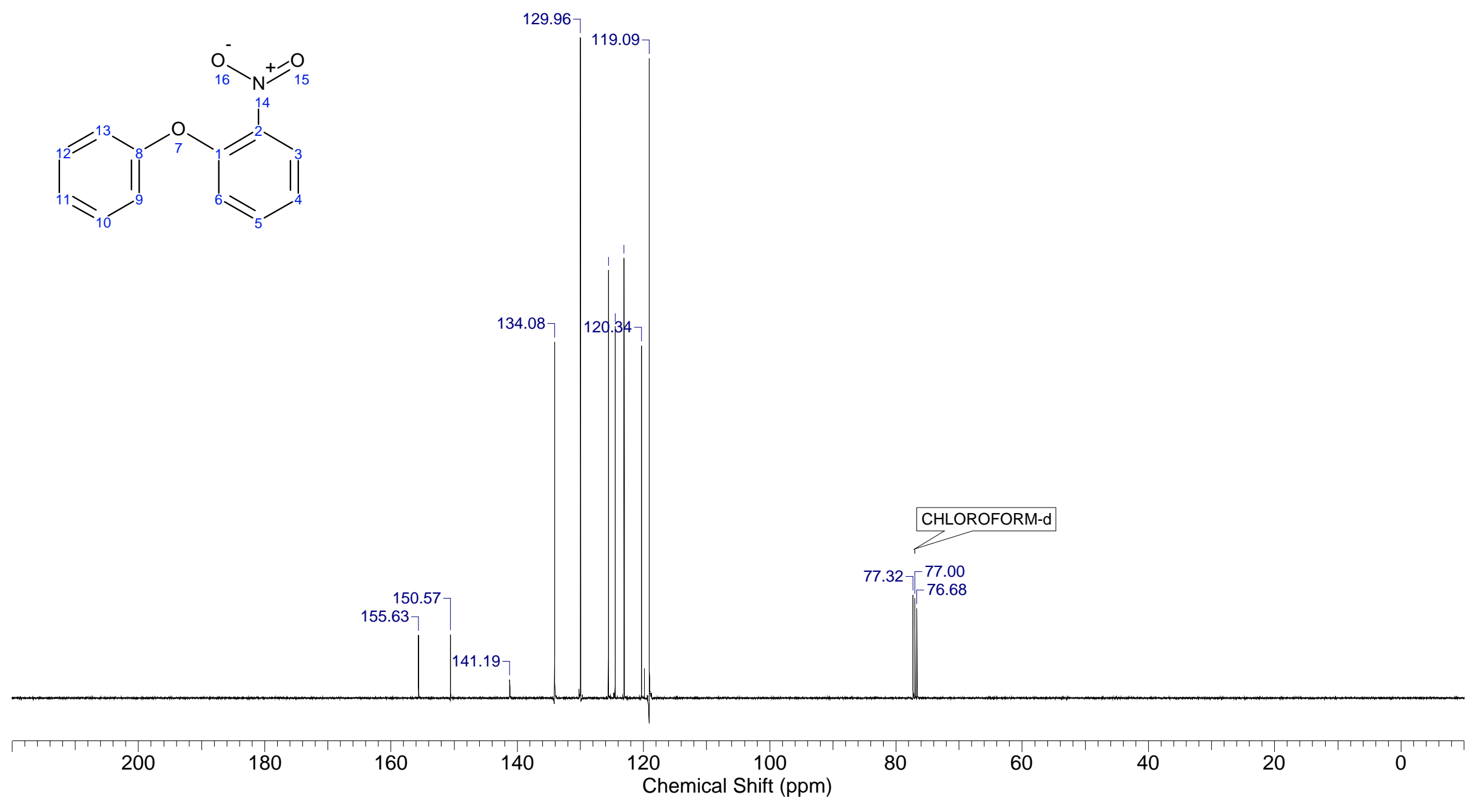



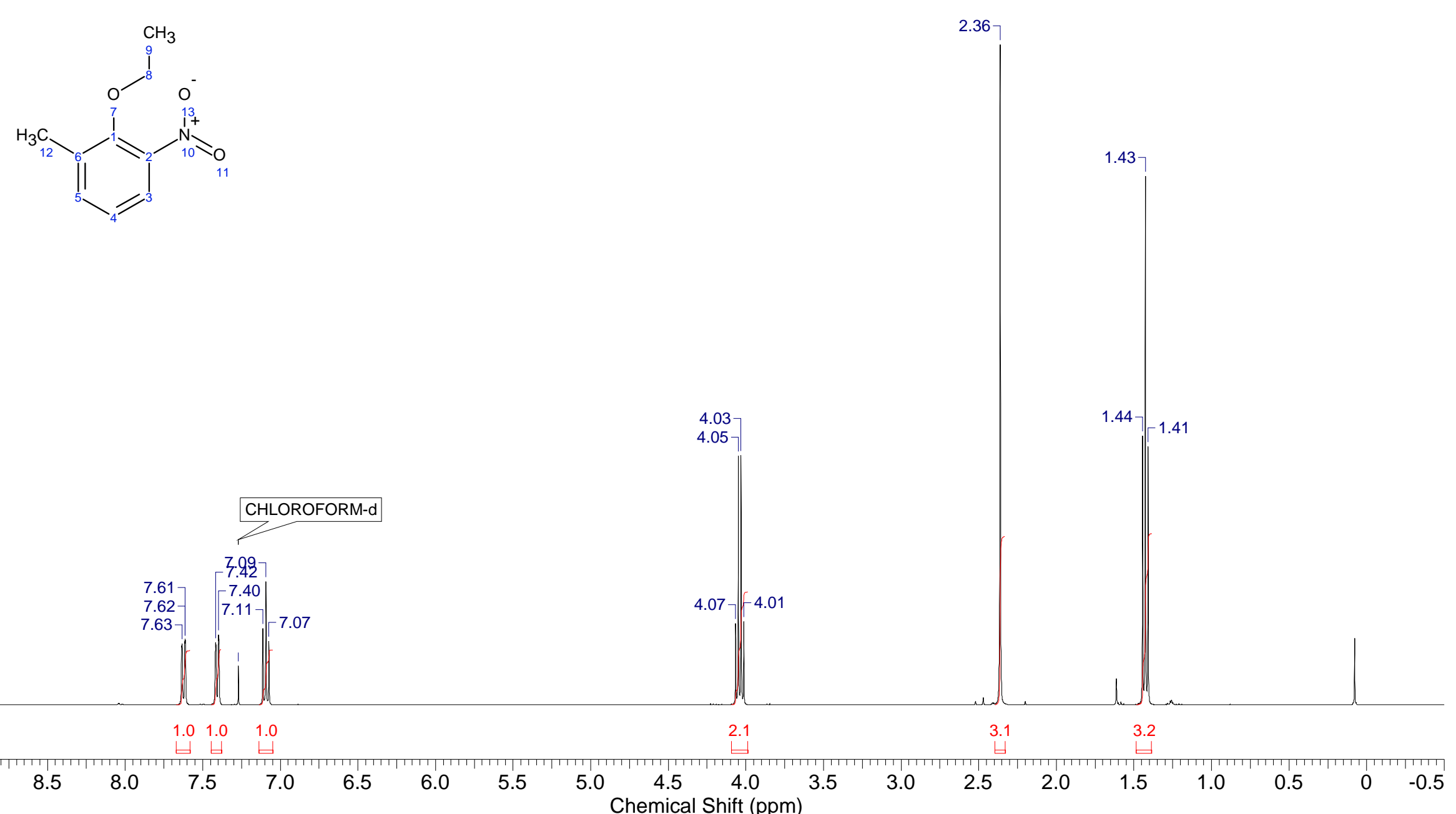


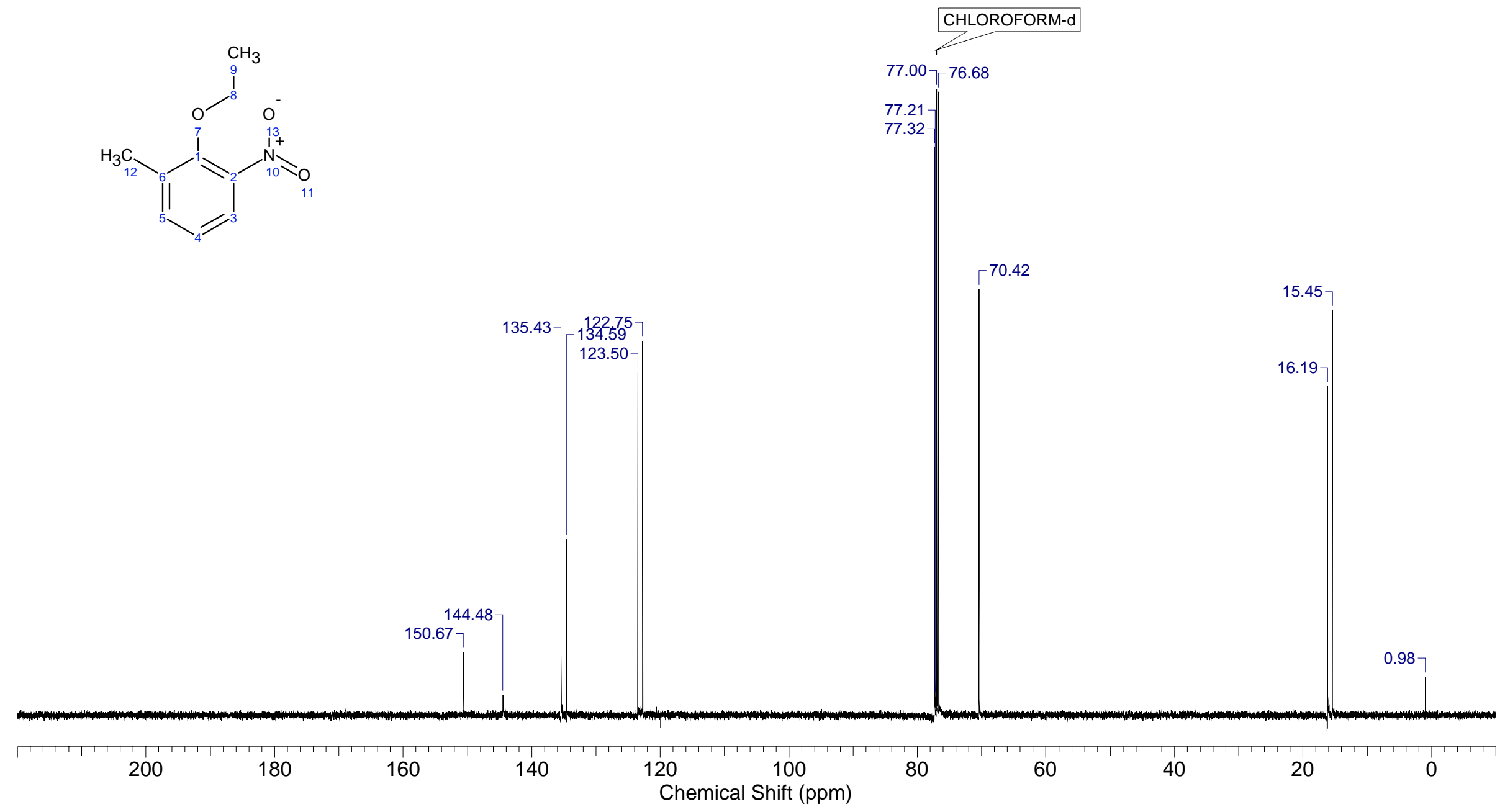



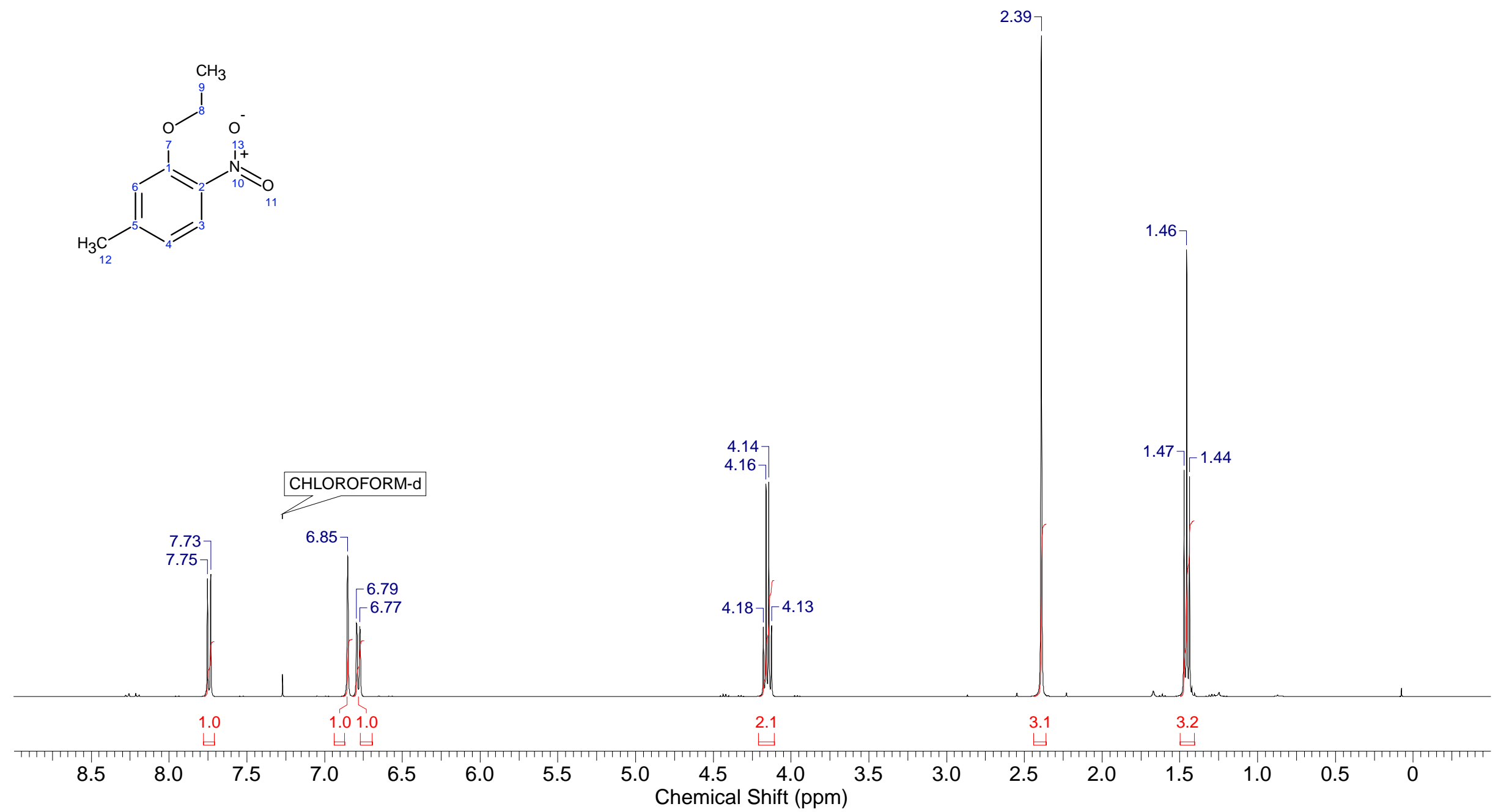


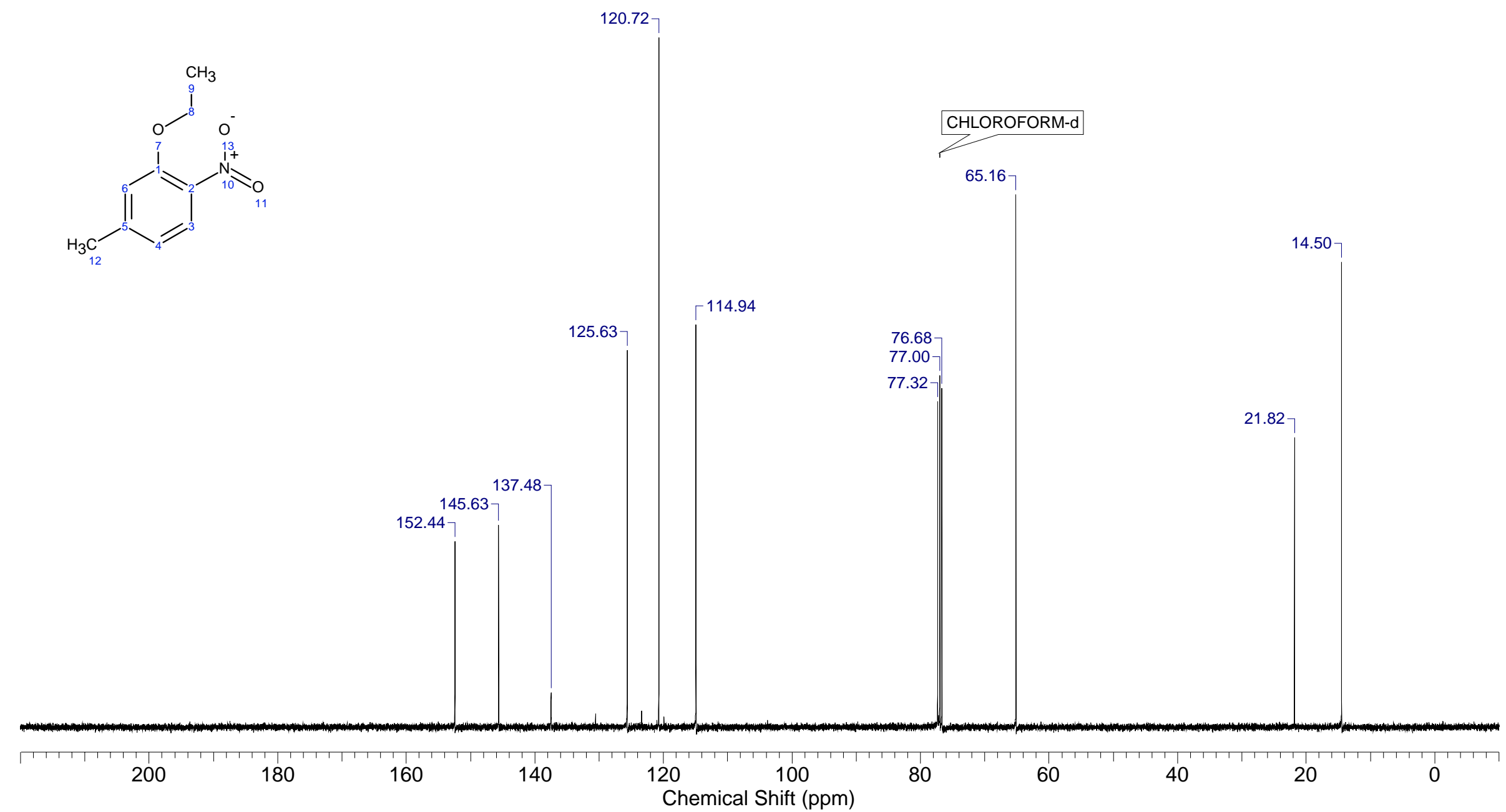



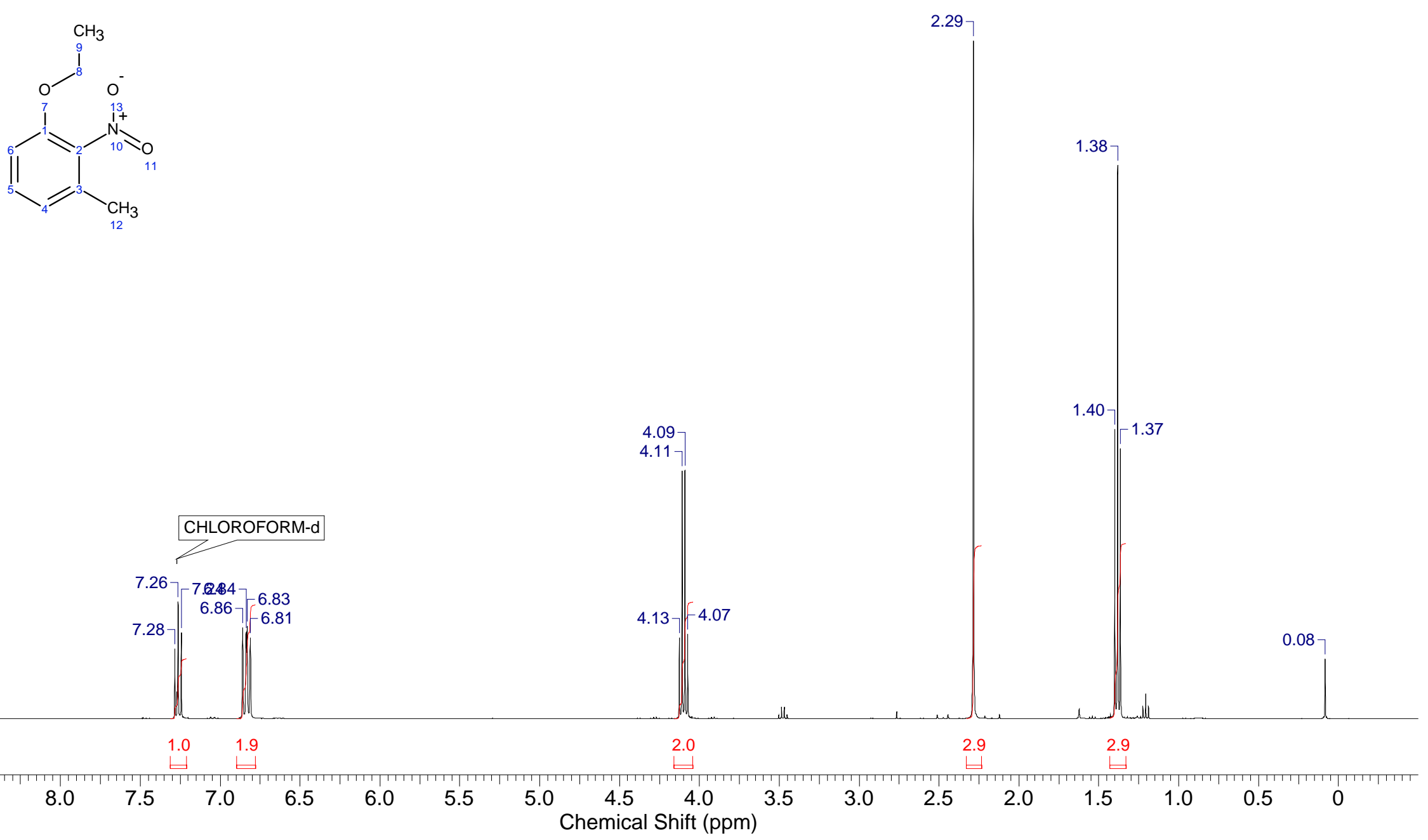


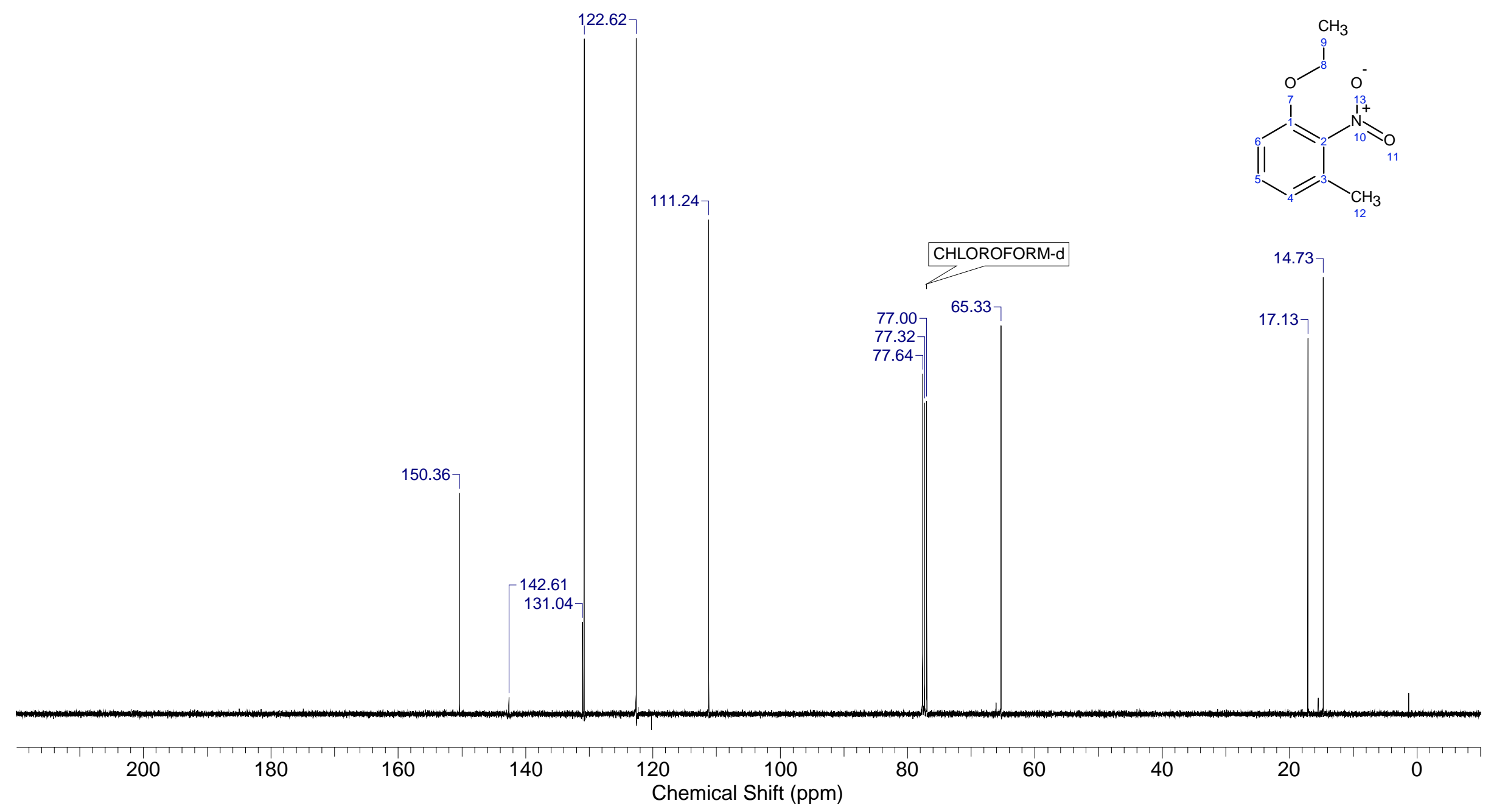




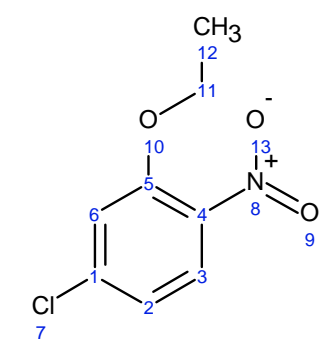

1.49

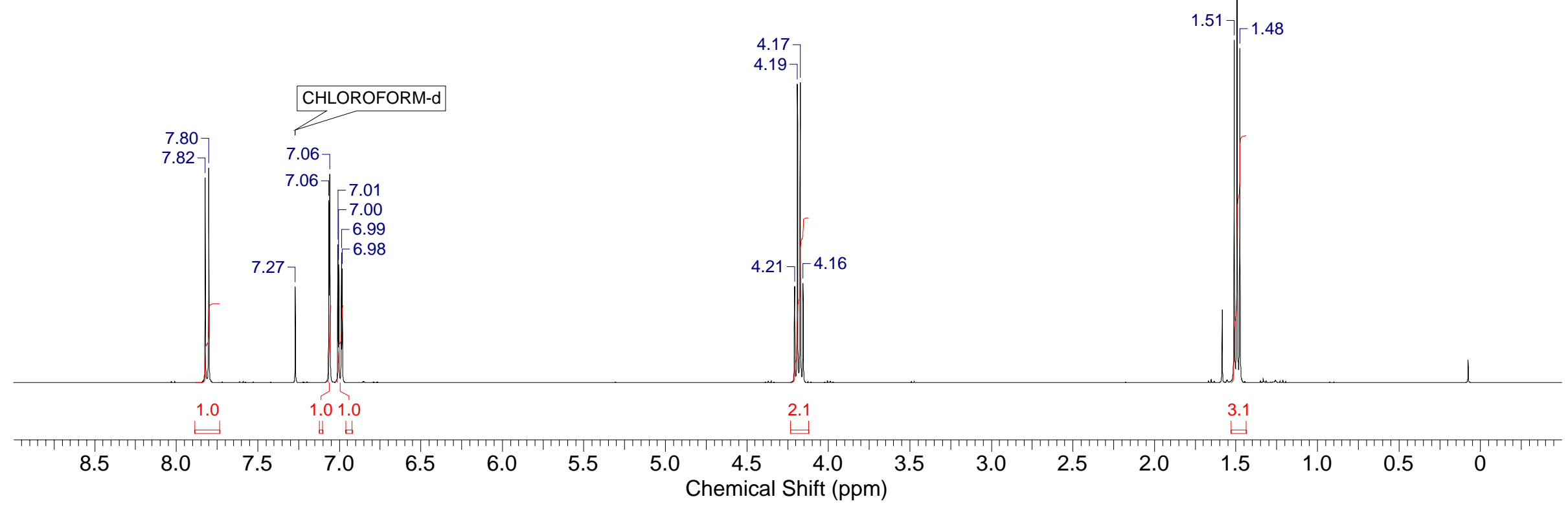



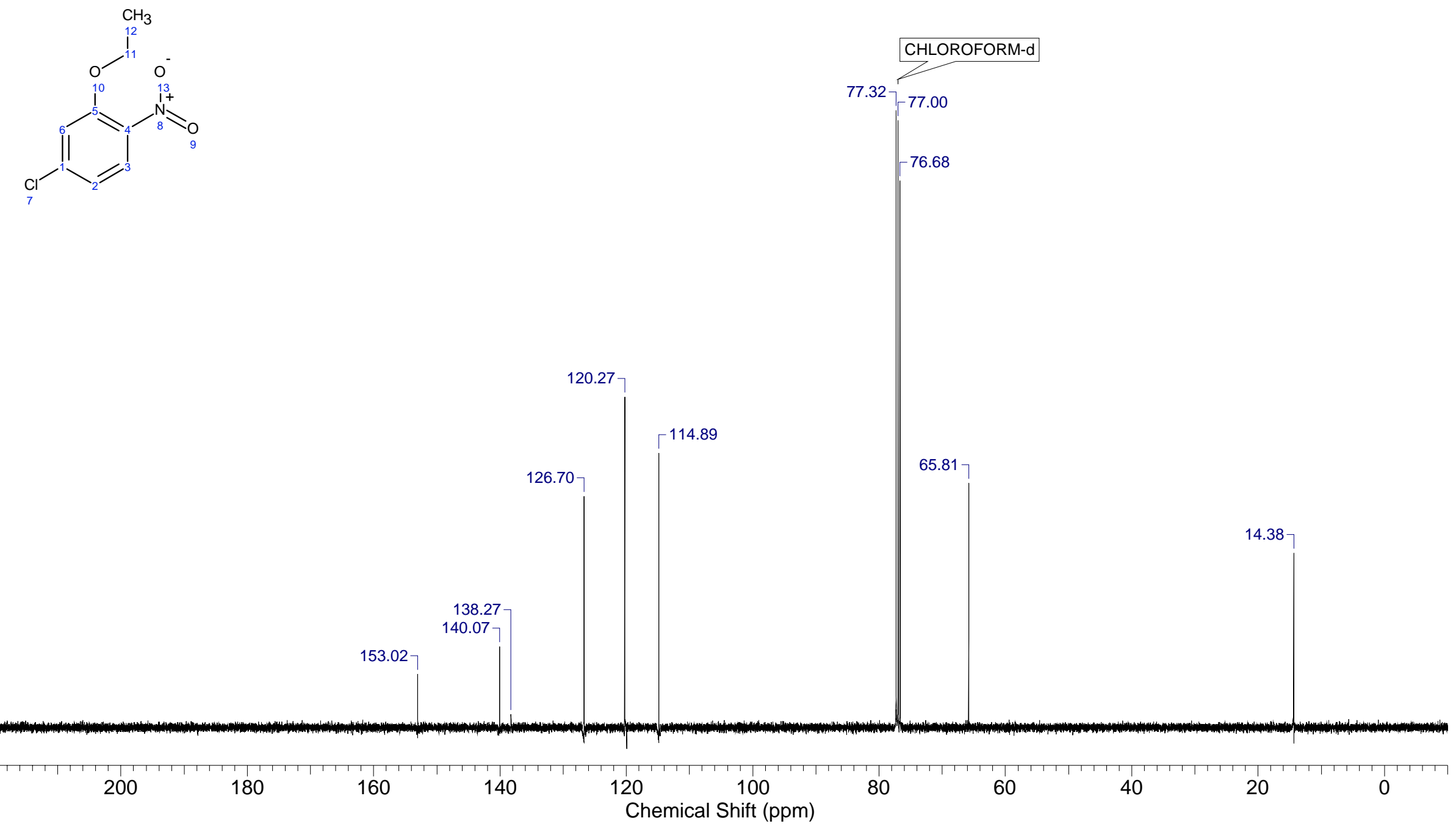

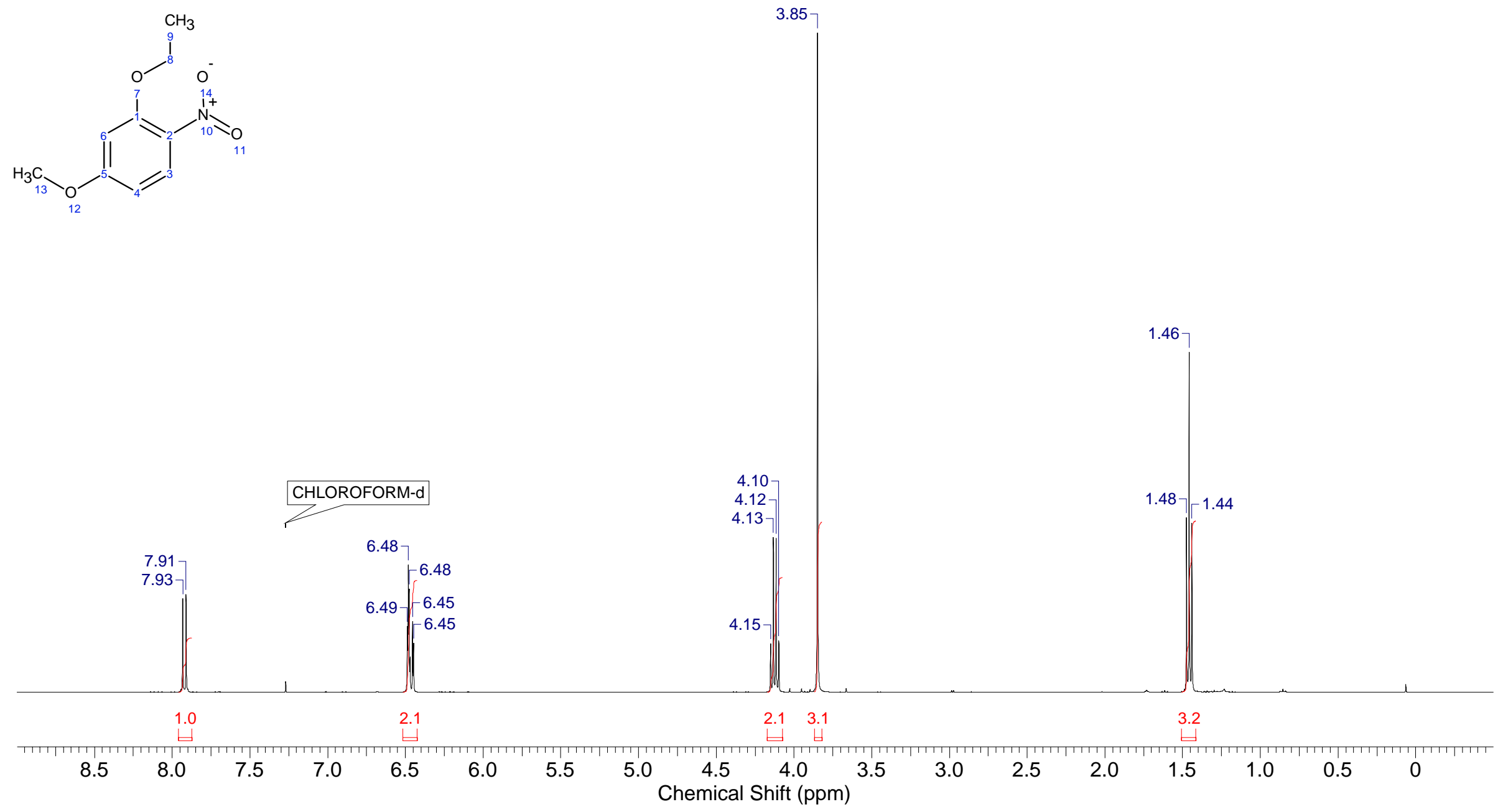
2-Ethoxy-4-methoxy-1-nitrobenzene (3fb)

${ }^{13} \mathrm{C}$ NMR, $101 \mathrm{MHz}, \mathrm{CDCl}_{3}$<smiles>CCOc1cc(OC)ccc1[N+](=O)[O-]</smiles>

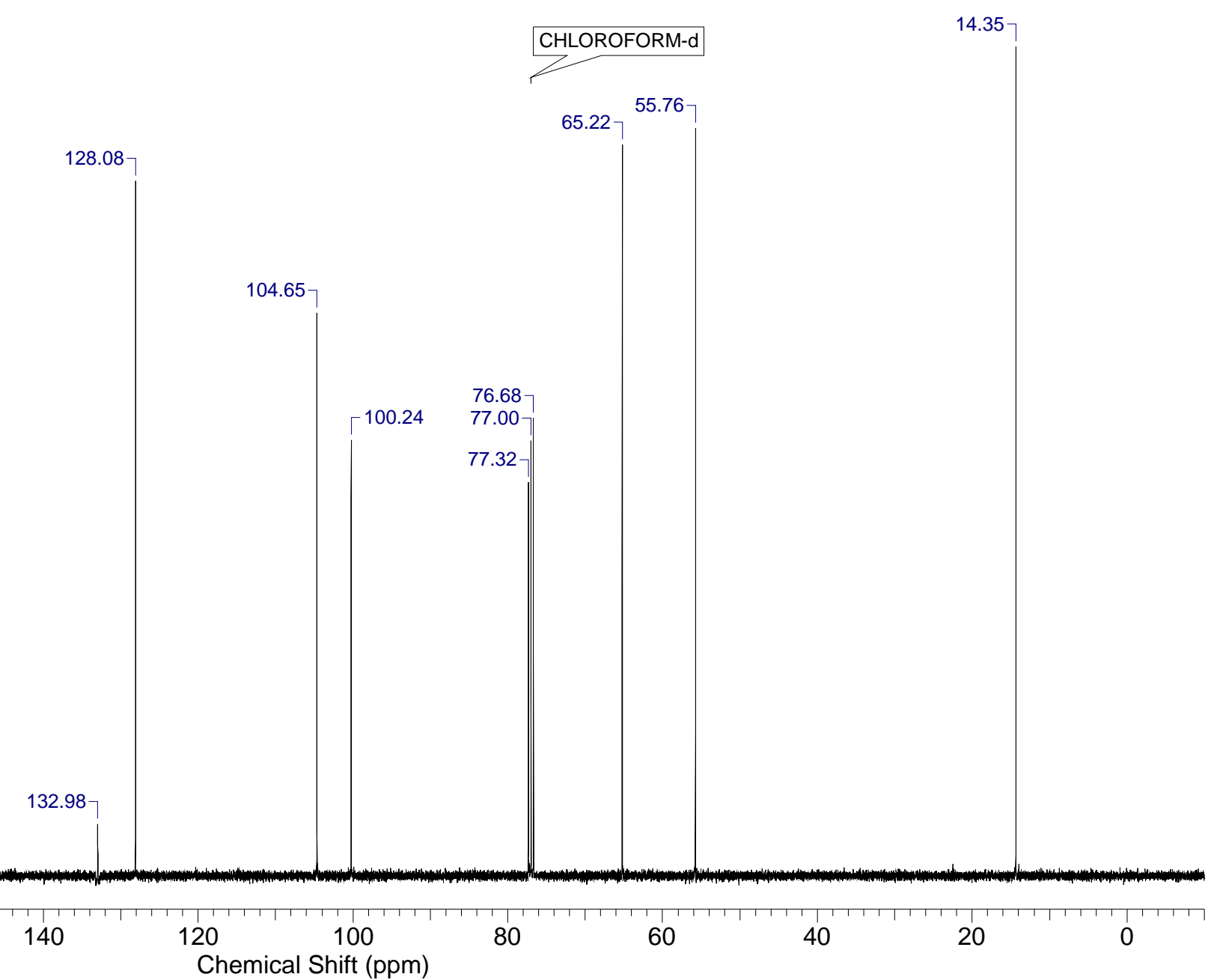


1,2-Dimethoxybenzene (3ga)

${ }^{1} \mathrm{H}$ NMR, $400 \mathrm{MHz}, \mathrm{CDCl}_{3}$<smiles></smiles>

3.88

CHLOROFORM-d

$6.91-6.91$

$6.92-6.90$

$6.93] 6.89$

$6.93]-6.88$

$6.94-6.87$

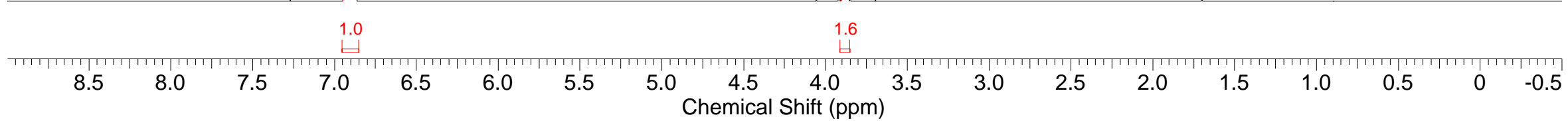




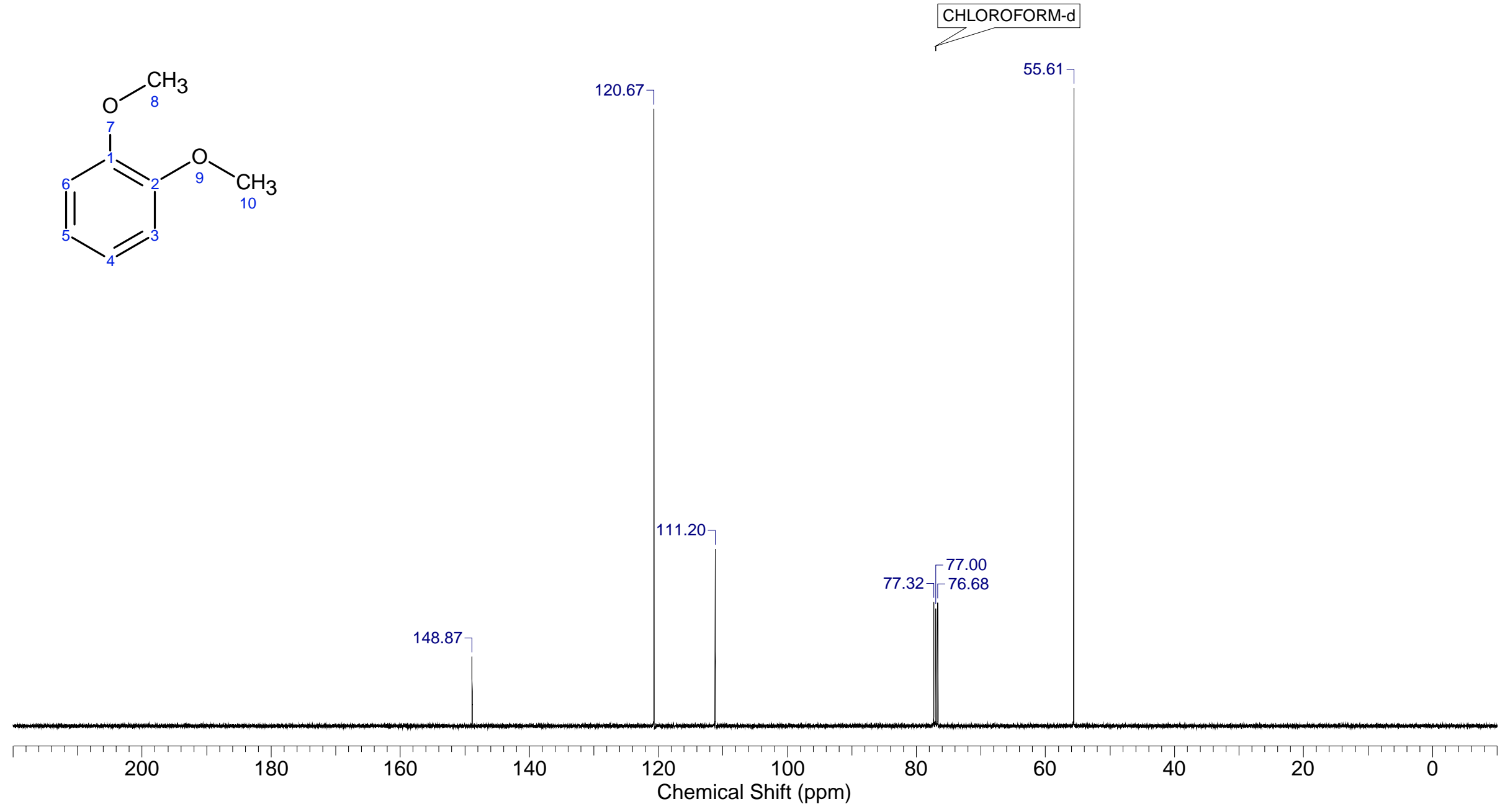


${ }^{1} \mathrm{H}$ NMR, $400 \mathrm{MHz}, \mathrm{CDCl}_{3}$
1,2,3-Trimethoxybenzene (3ha)

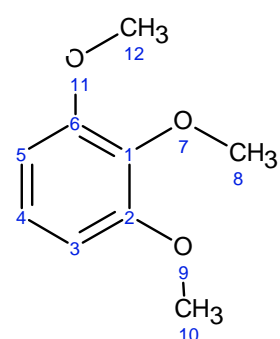

3.85

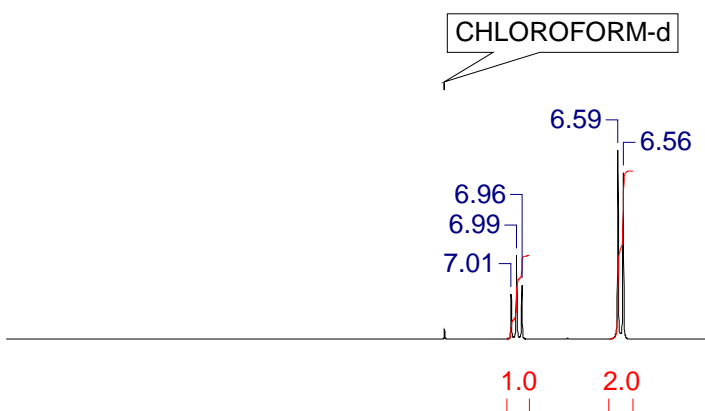

$\left.{ }^{3.85}\right\urcorner$

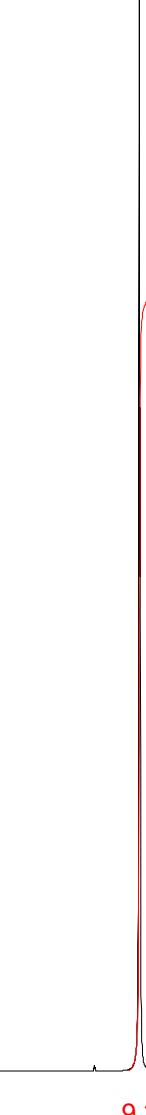




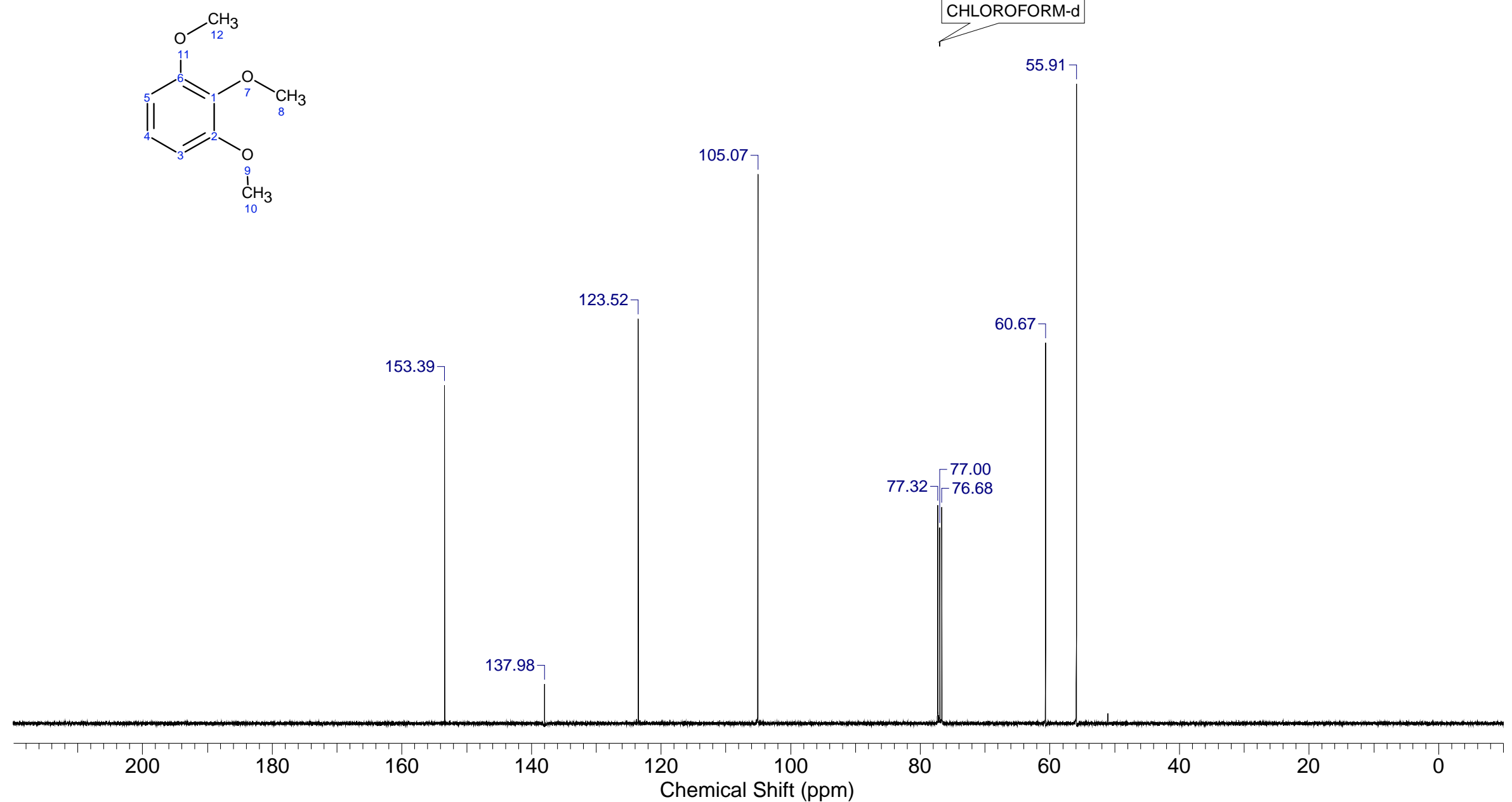



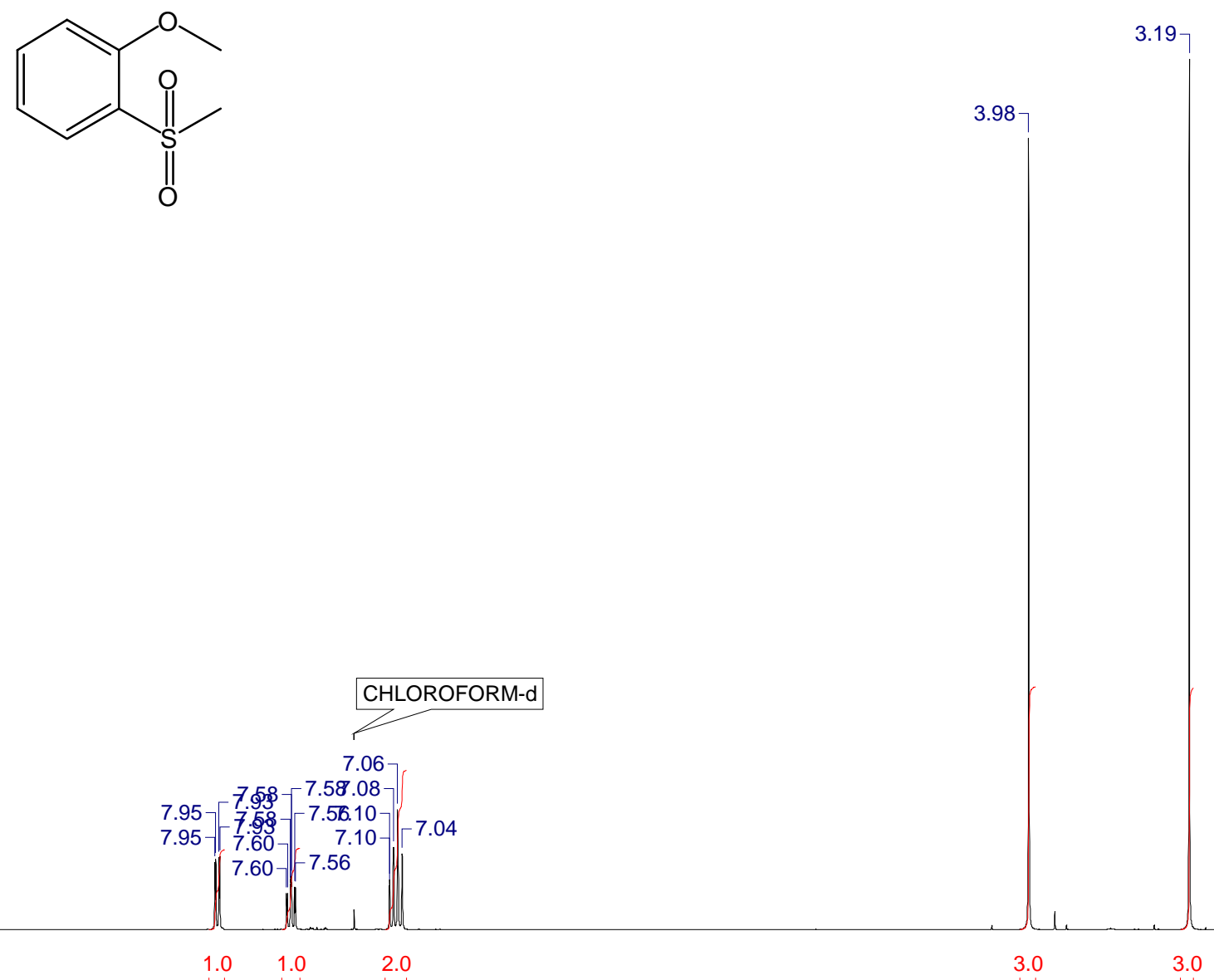

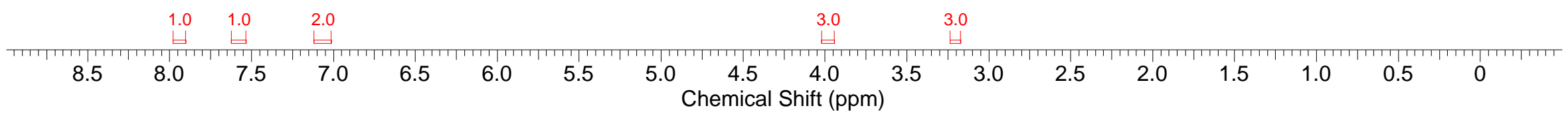


1-Methoxy-2-(methylsulfonyl)benzene (3ia)

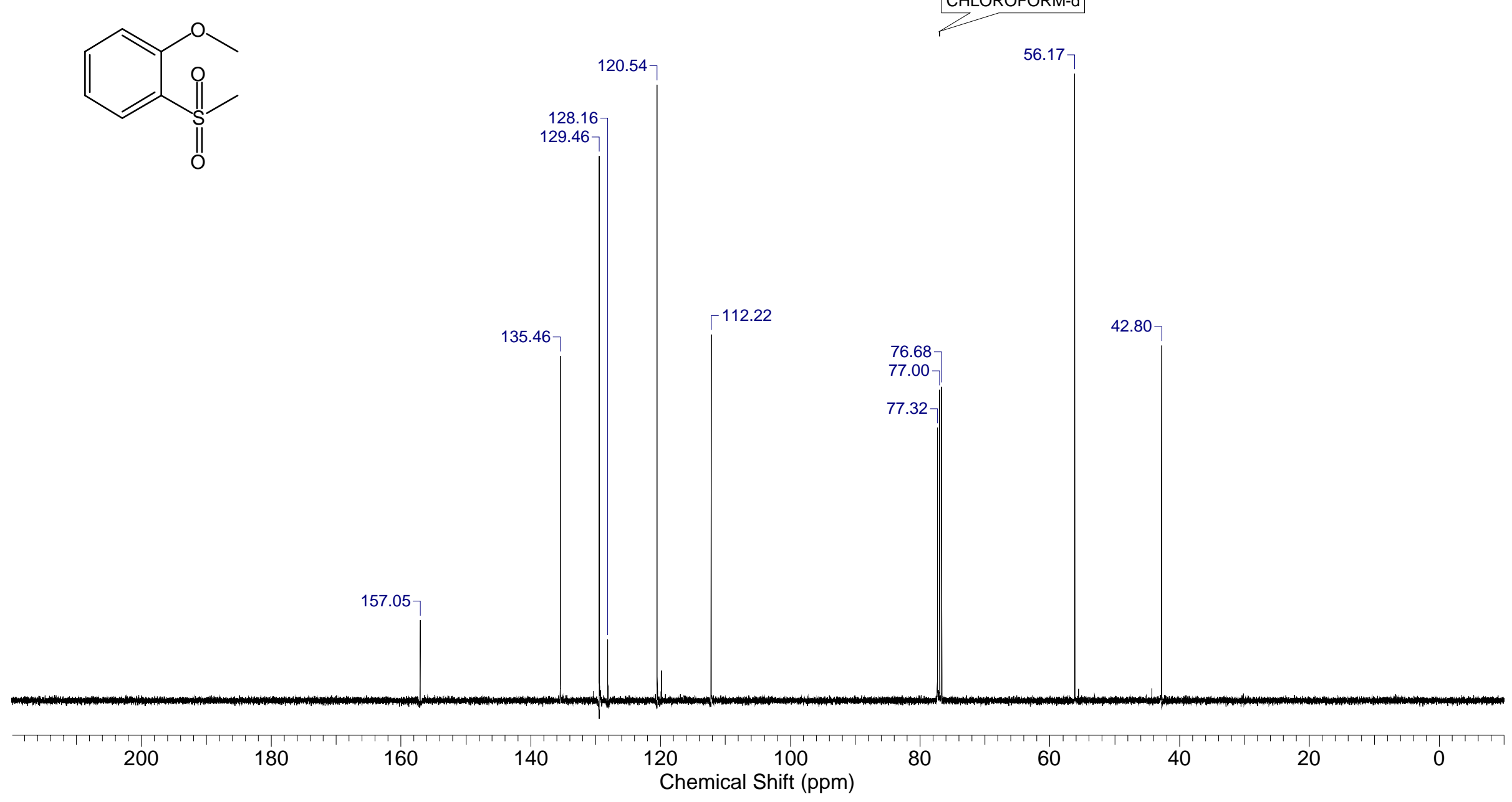

${ }^{13} \mathrm{C}$ NMR, $101 \mathrm{MHz}, \mathrm{CDCl}_{3}$

CHLOROFORM-d 


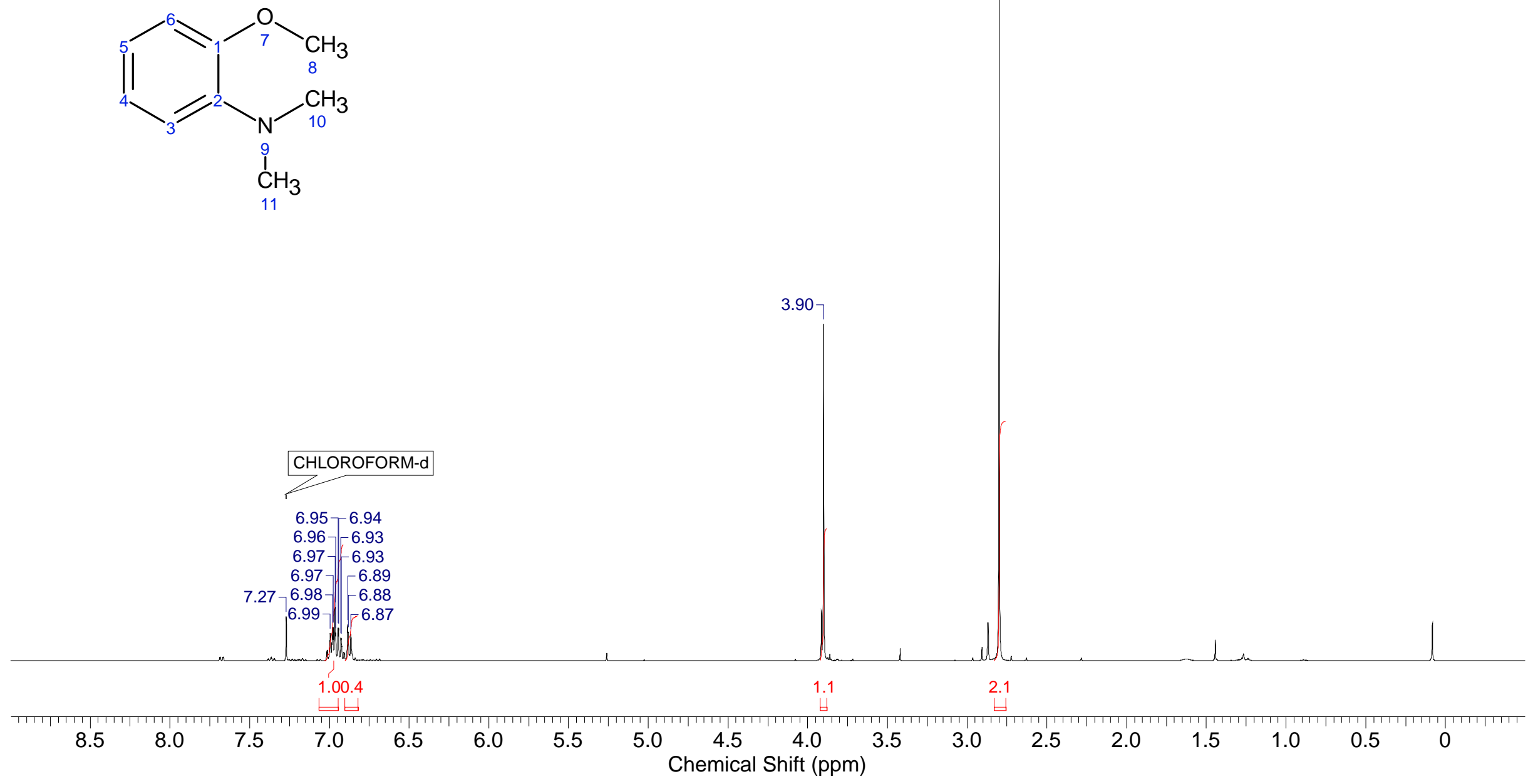




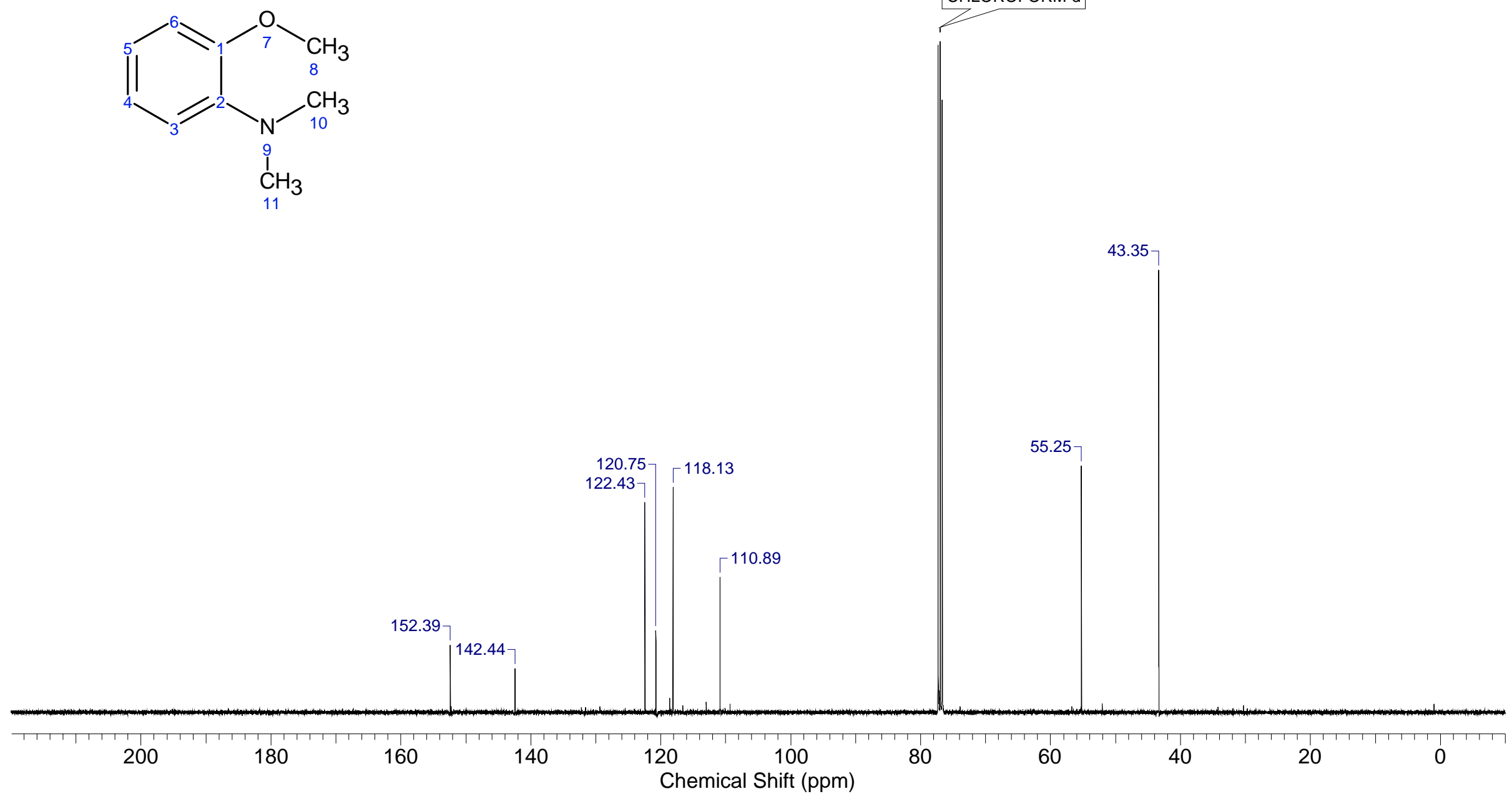

CHLOROFORM-d 
1-Chloro-2-ethoxy-4-nitrobenzene (3kb)

${ }^{1} \mathrm{H}$ NMR, $400 \mathrm{MHz}, \mathrm{CDCl}_{3}$

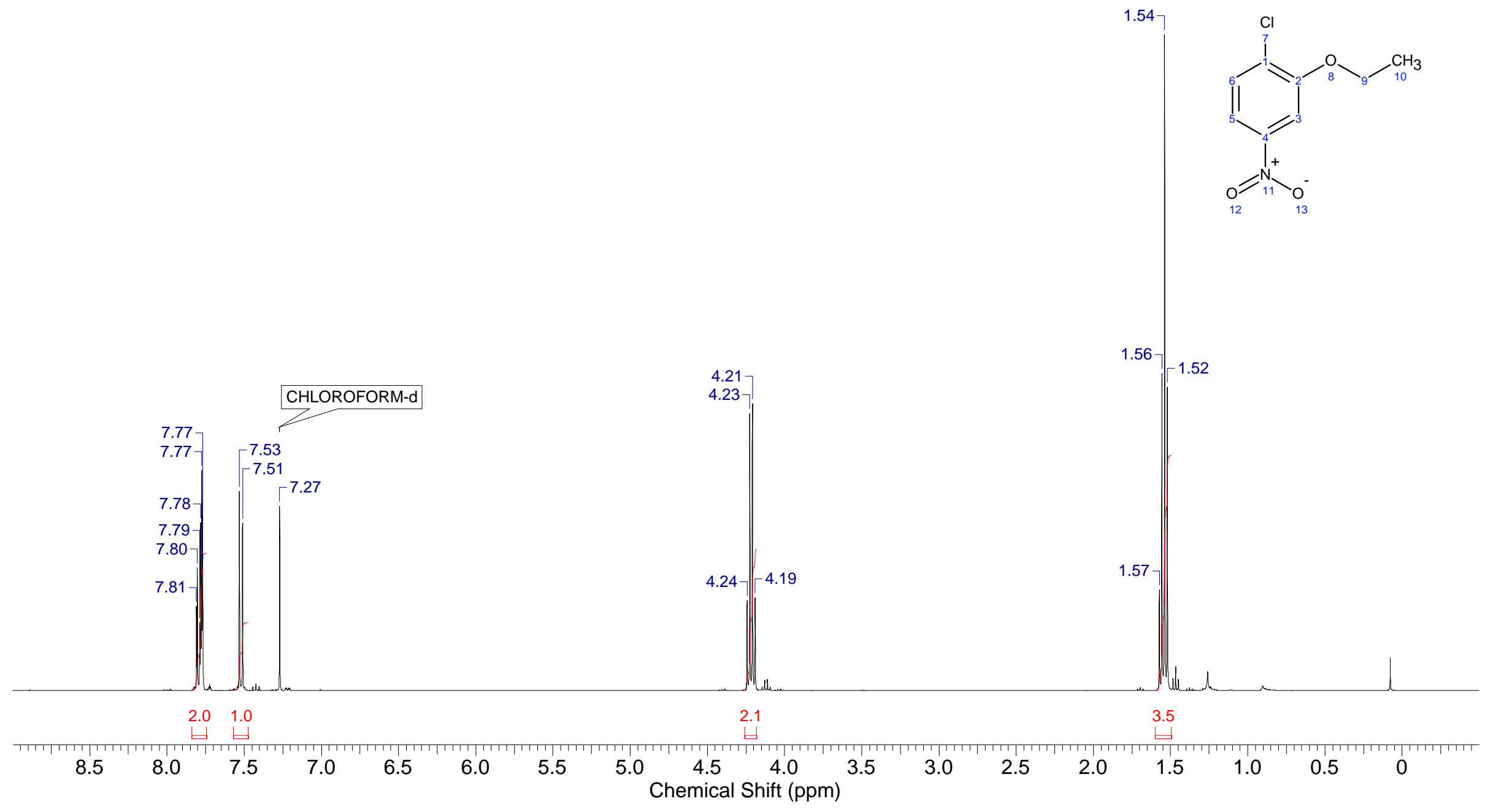


1-Chloro-2-ethoxy-4-nitrobenzene (3kb)

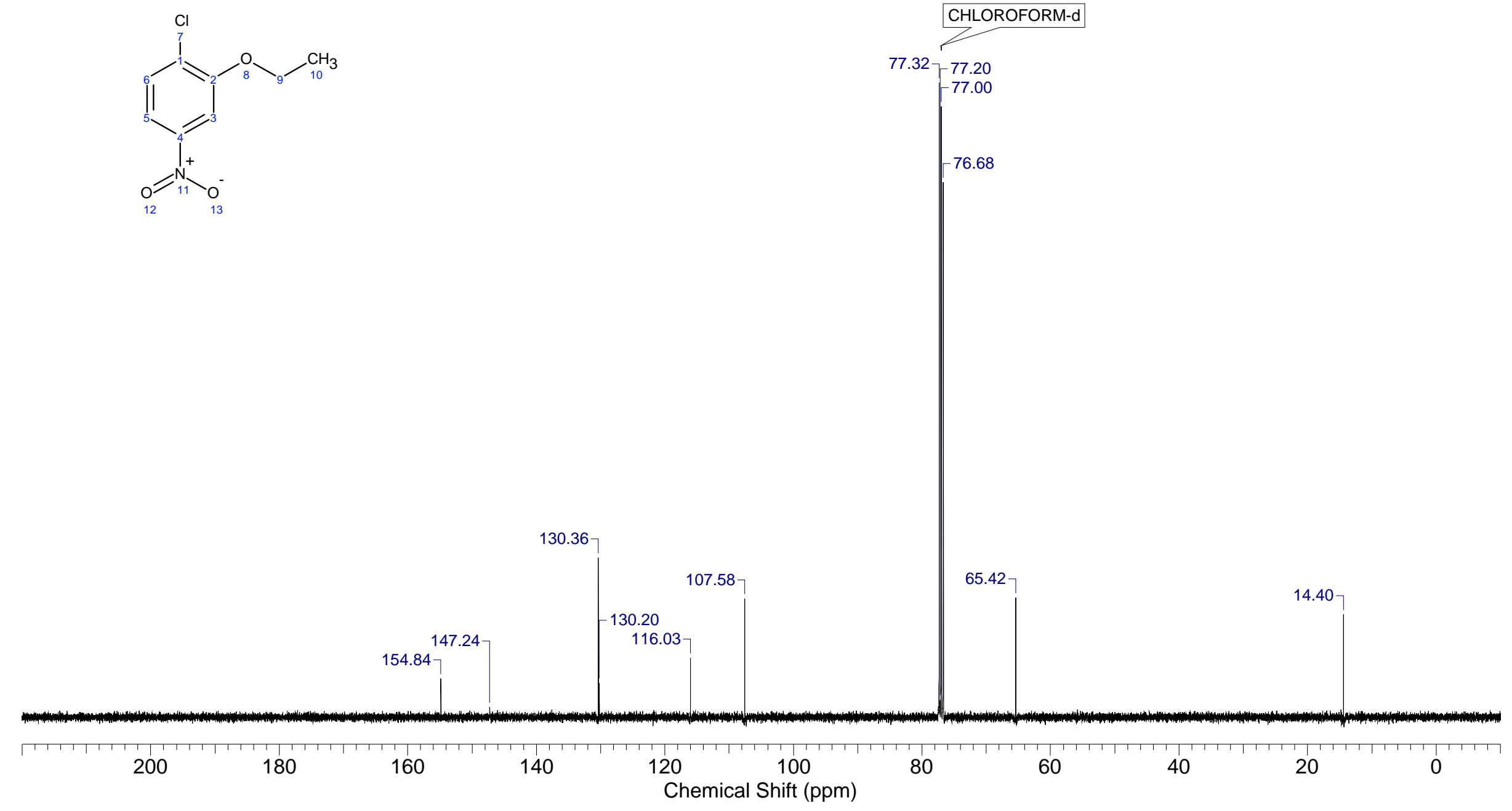

${ }^{13} \mathrm{C}$ NMR, $101 \mathrm{MHz}, \mathrm{CDCl}_{3}$ 
1-Butoxy-2-fluorobenzene (3ld)

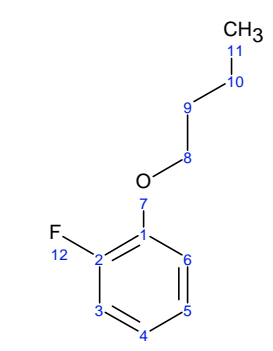

${ }^{1} \mathrm{H}$ NMR, $400 \mathrm{MHz}, \mathrm{CDCl}_{3}$

$$
1.02\urcorner
$$

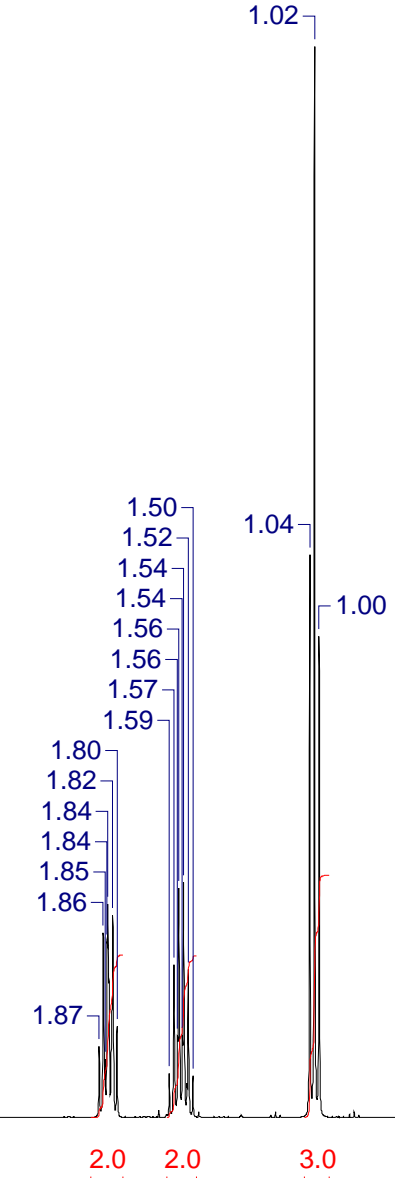

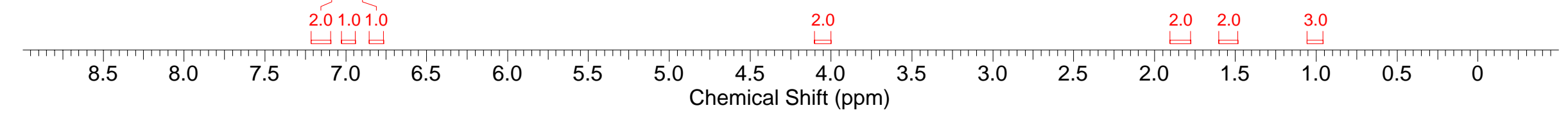


1-Butoxy-2-fluorobenzene (3ld)

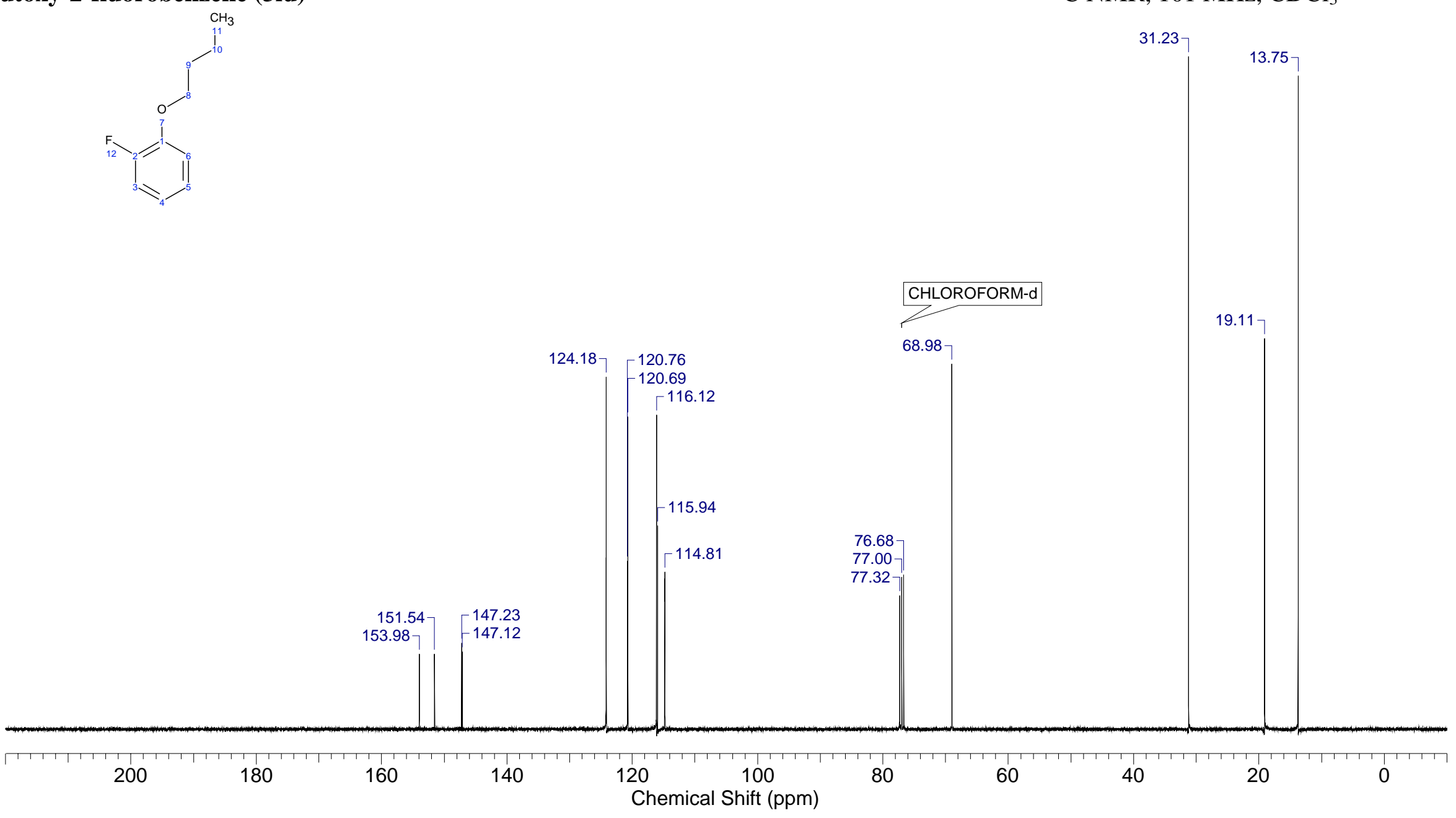


1-Methoxy-2-trifluoromethylbenzene (3ma)

${ }^{1} \mathrm{H}$ NMR, $400 \mathrm{MHz}, \mathrm{CDCl}_{3}$<smiles>COc1ccccc1[PH](F)(F)F</smiles>

CHLOROFORM-d
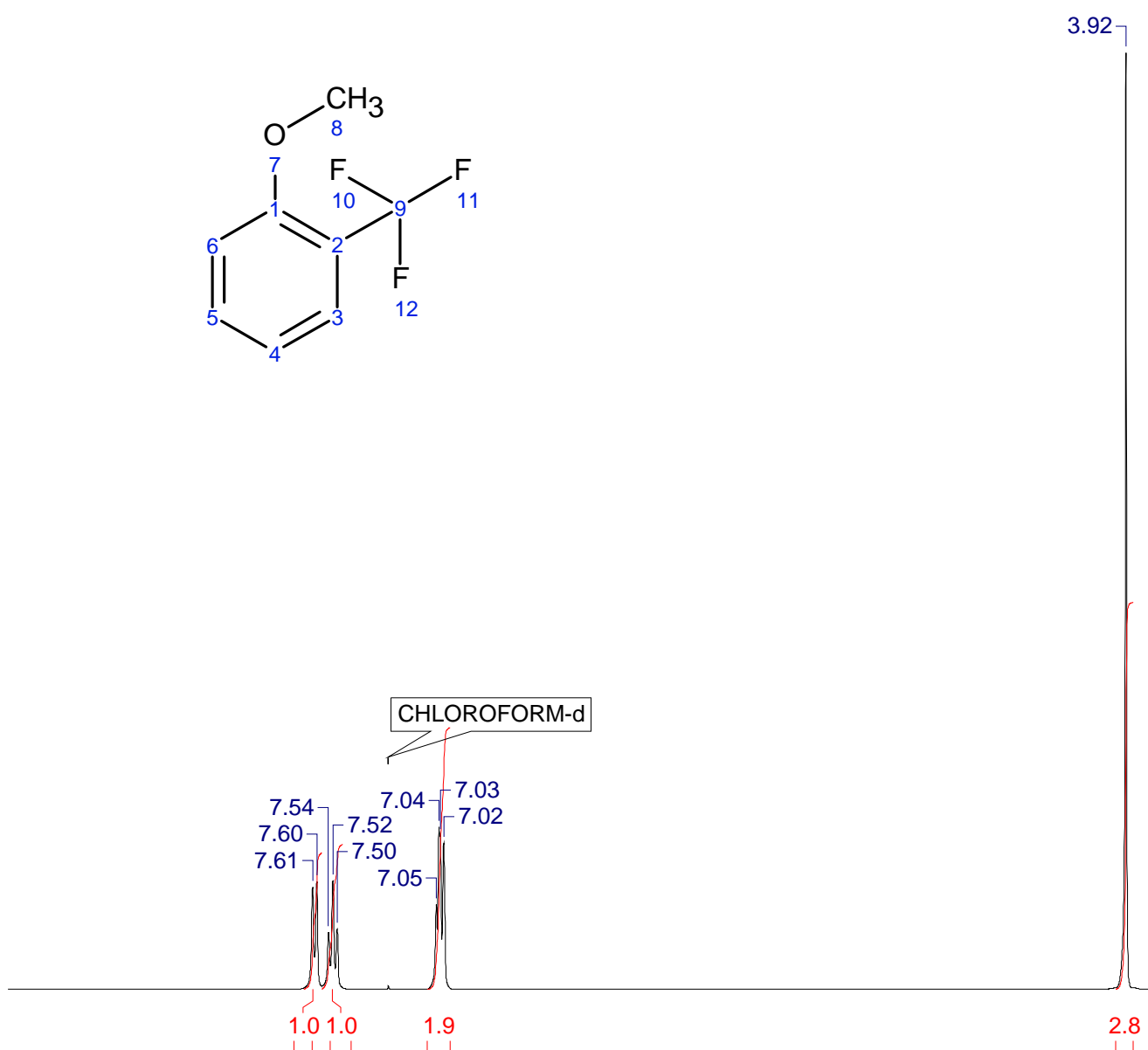

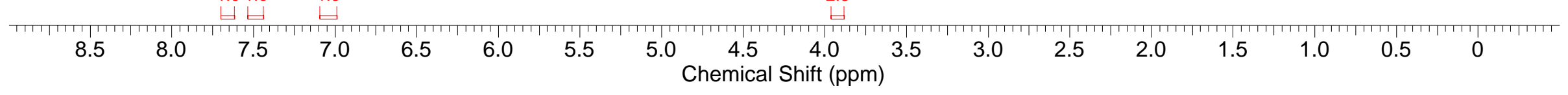


1-Methoxy-2-trifluoromethylbenzene (3ma)

${ }^{13} \mathrm{C}$ NMR, $101 \mathrm{MHz}, \mathrm{CDCl}_{3}$

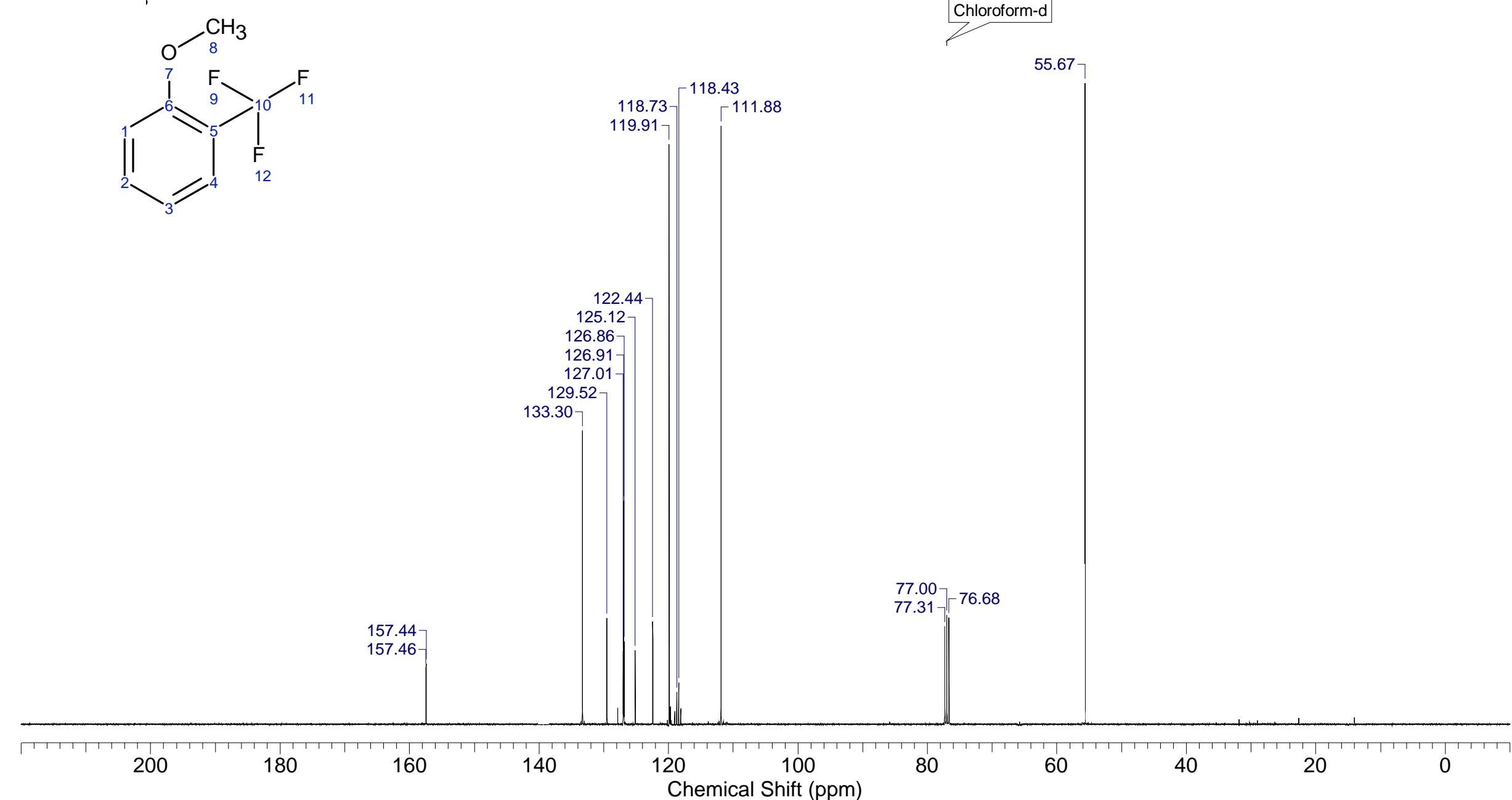

Chloroform-d 
2-Methoxy-1,1'-biphenyl (3na)

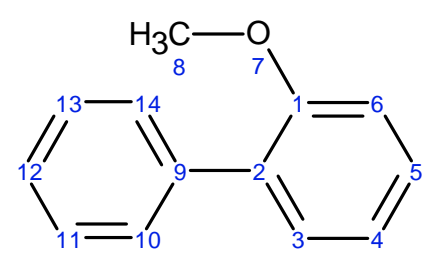

${ }^{1} \mathrm{H}$ NMR, $400 \mathrm{MHz}, \mathrm{CDCl}_{3}$

3.857

1.5

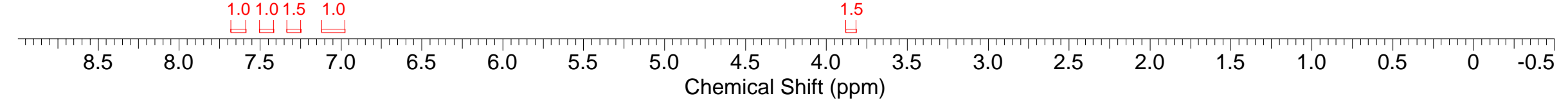


2-Methoxy-1,1'-biphenyl (3na)

${ }^{13} \mathrm{C}$ NMR, $101 \mathrm{MHz}, \mathrm{CDCl}_{3}$

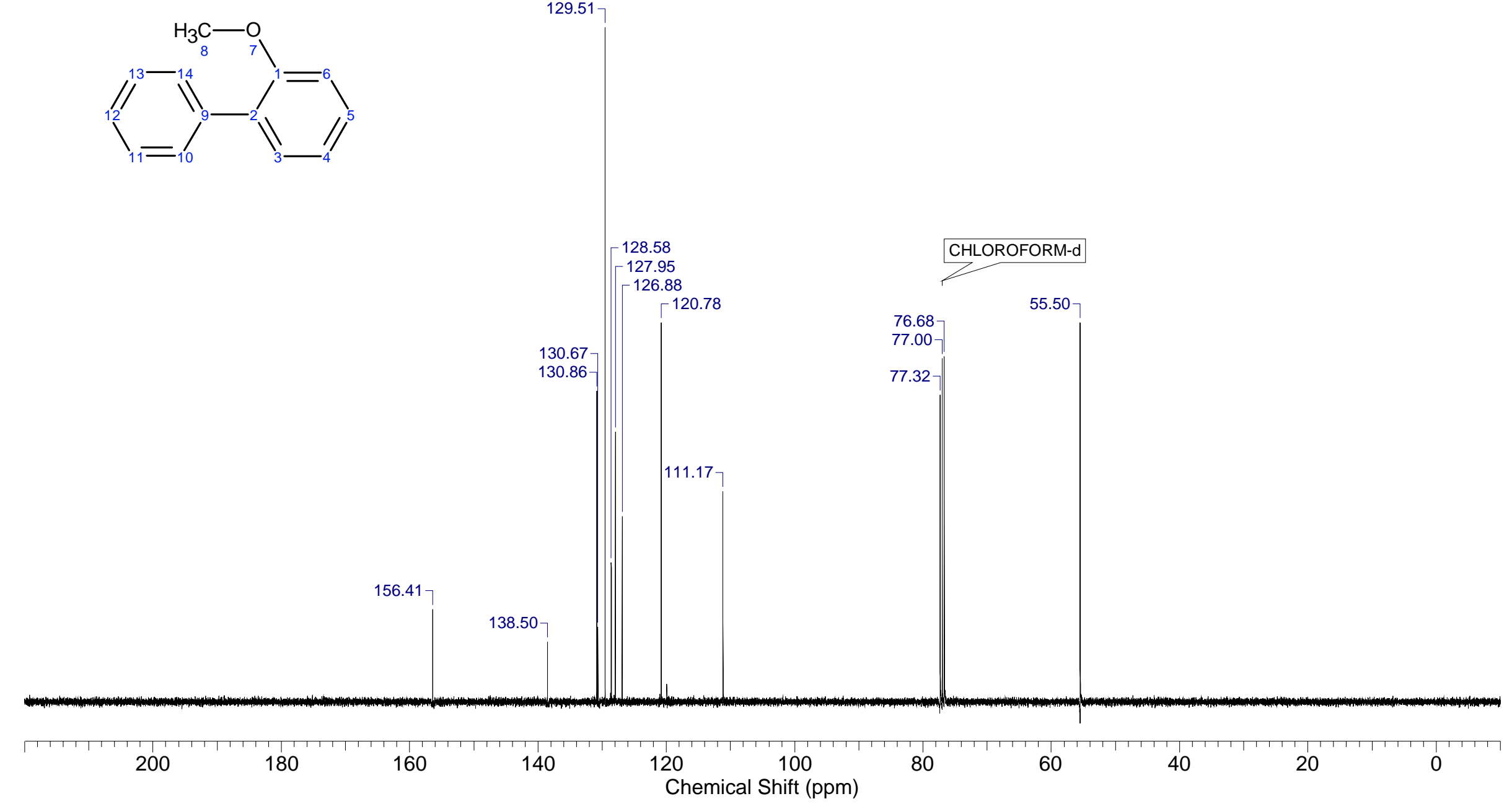

129.51 


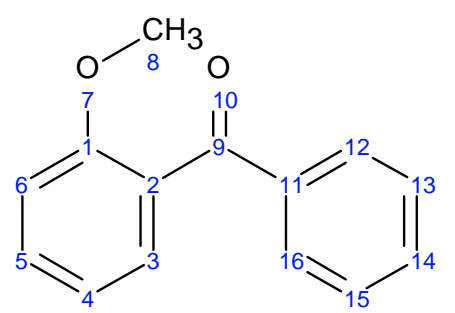

3.73

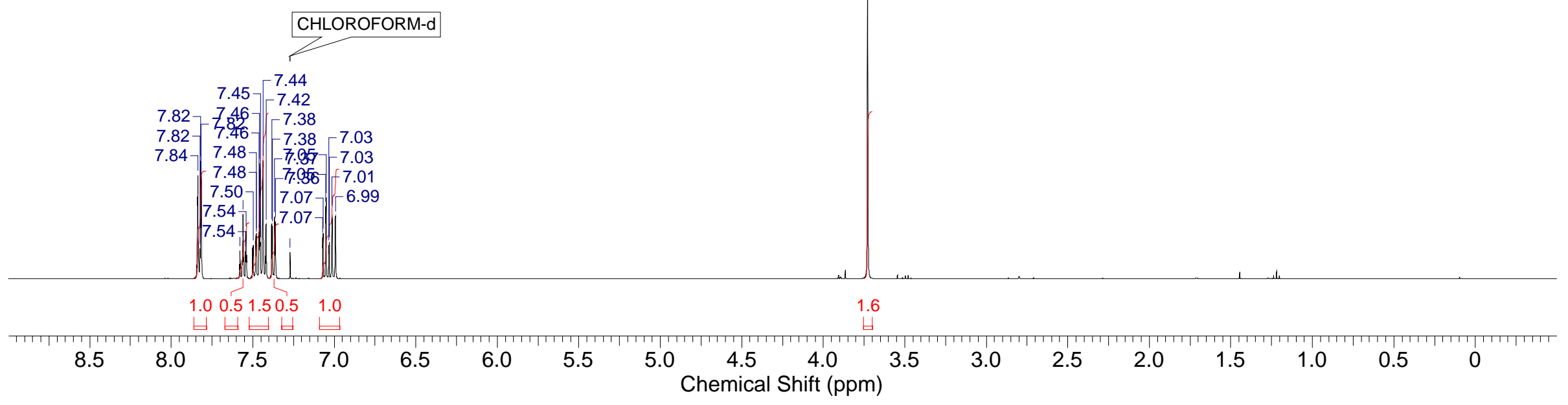




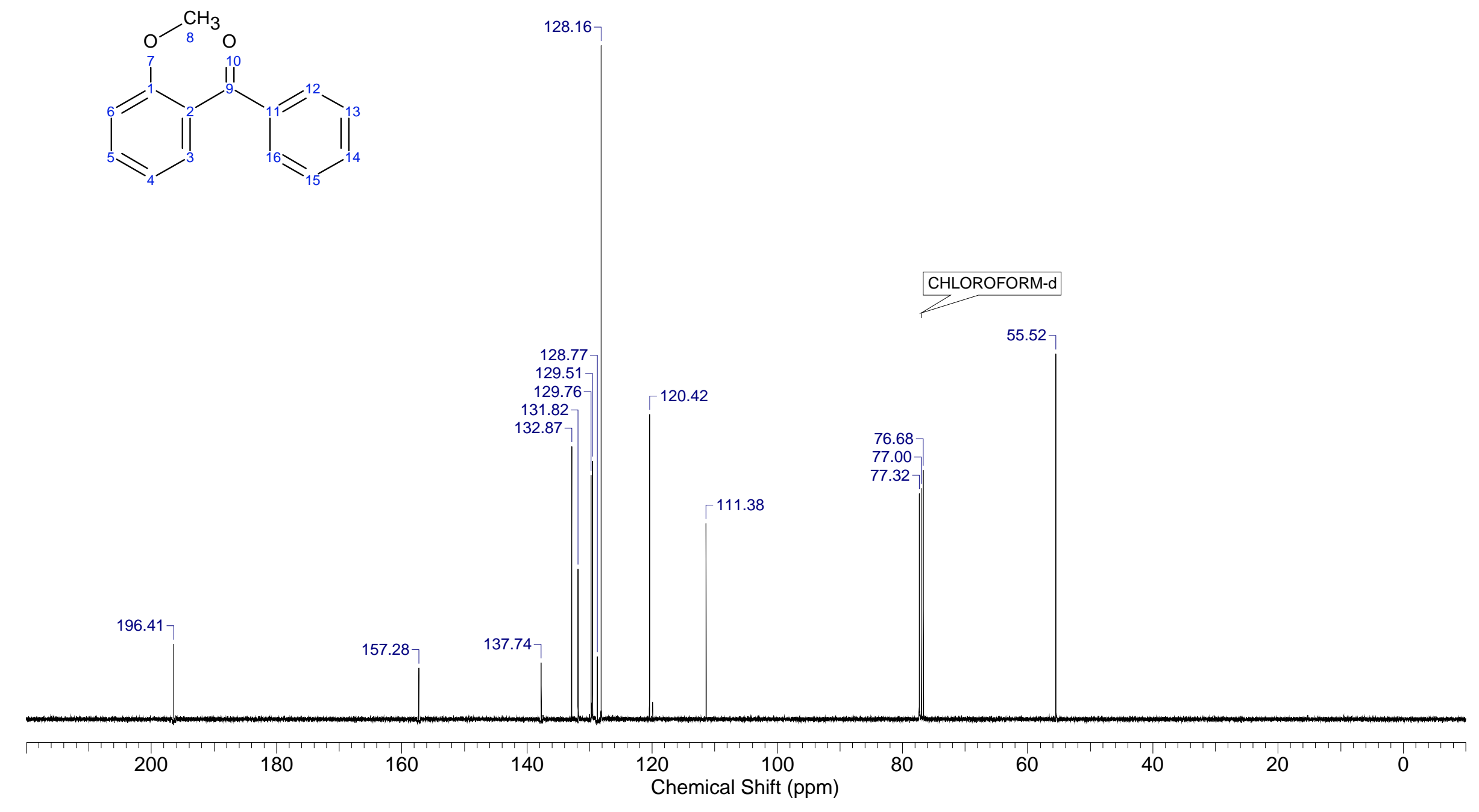

$\left.{ }^{128.16}\right\urcorner$ 
<smiles></smiles>

$$
3.87\urcorner
$$
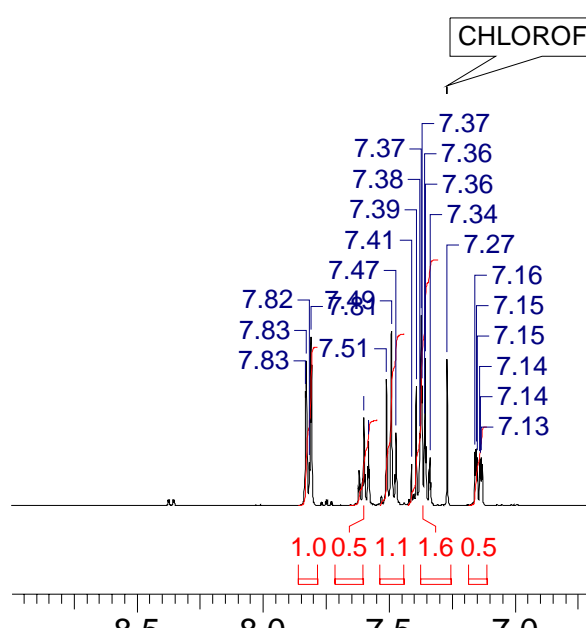

8.5

8.0

7.0

6.5

6.0

5.5

5.0

4.5

1.5 


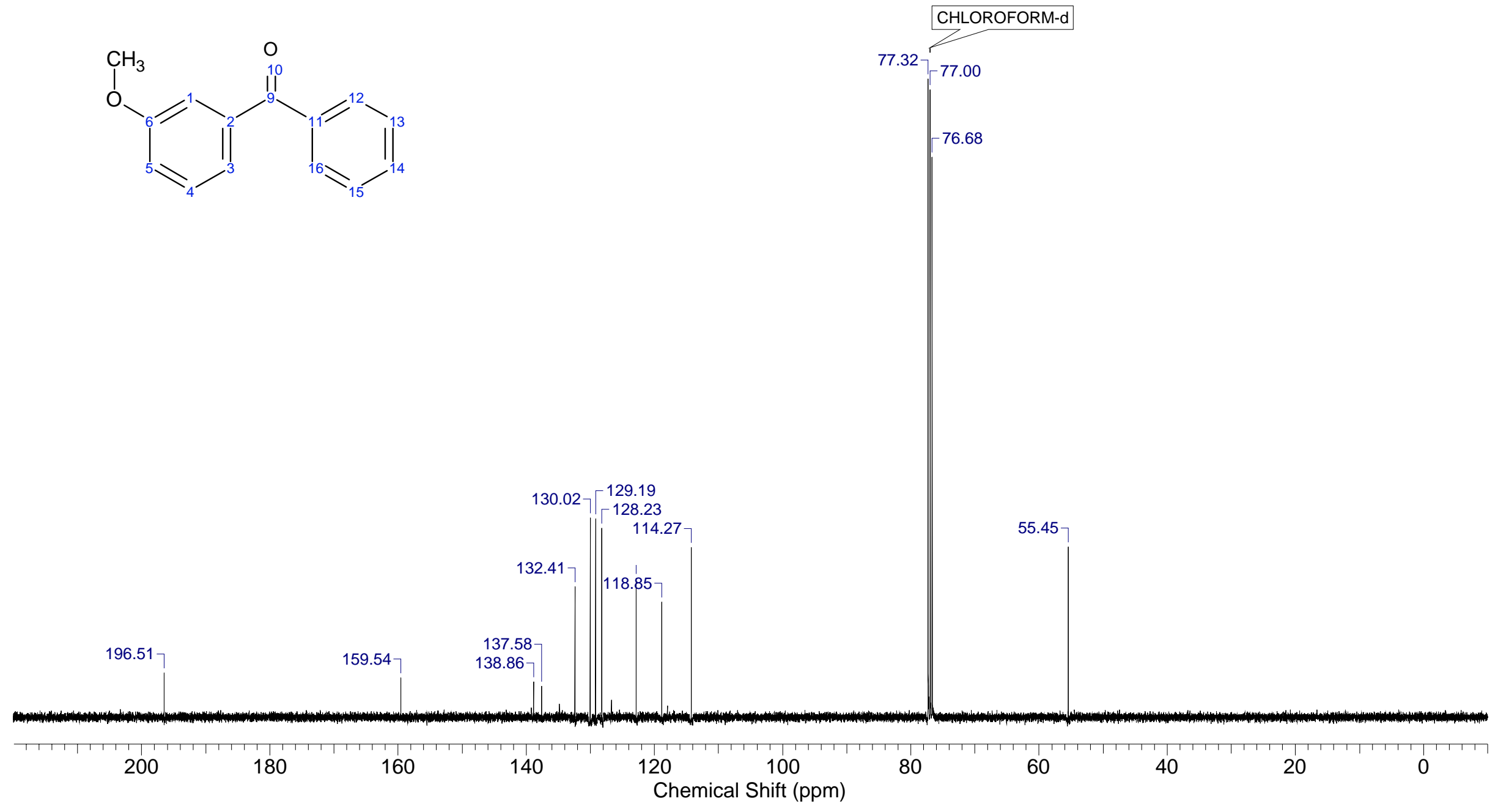



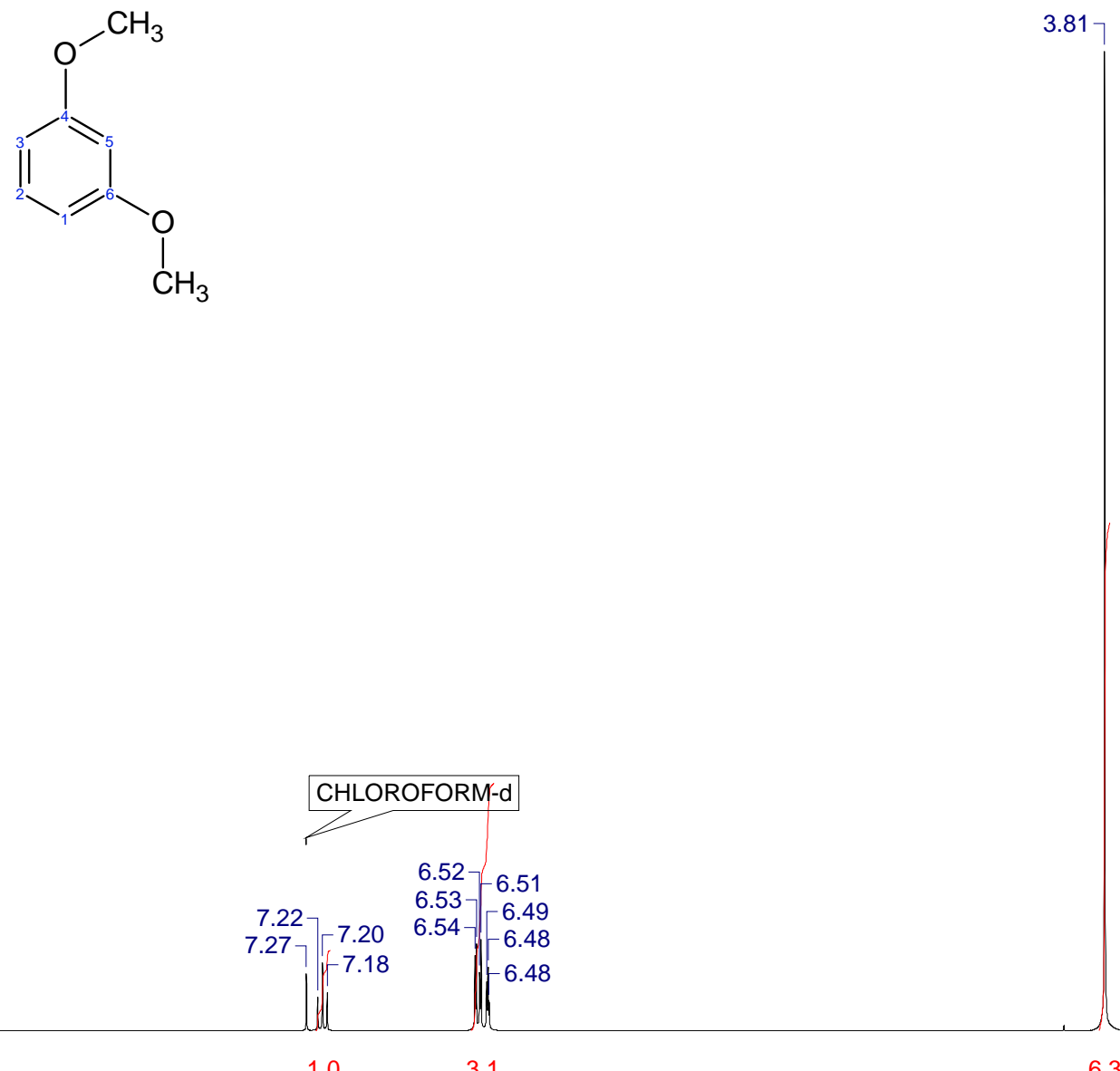

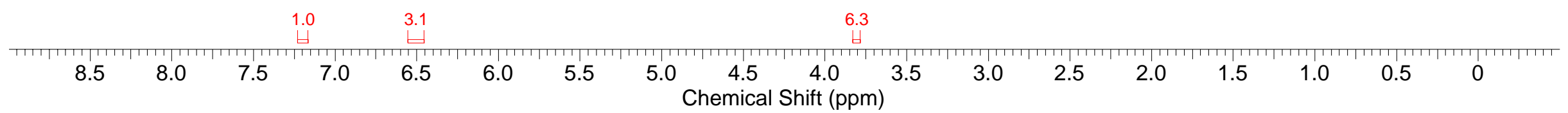


1,3-dimethoxybenzene (7)

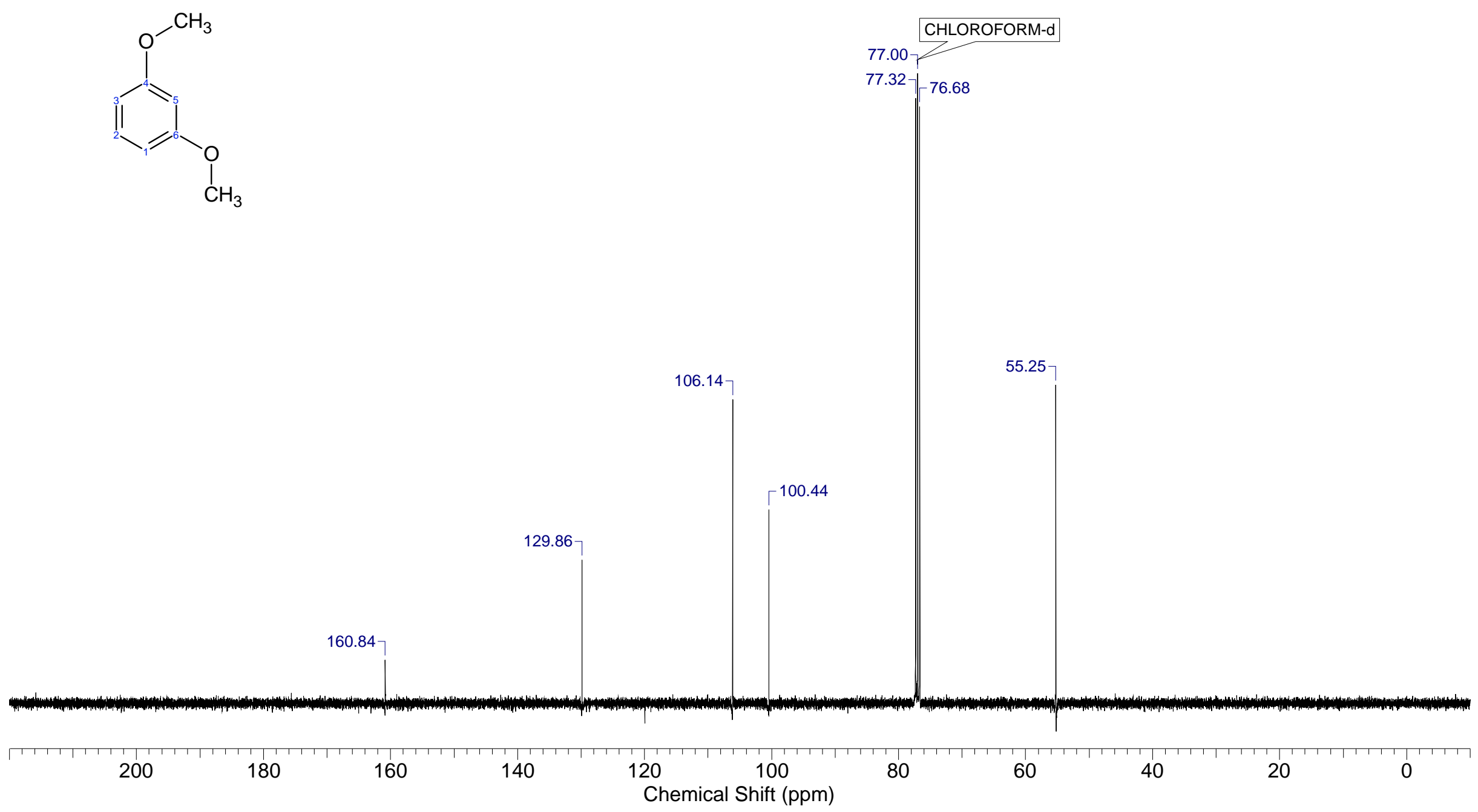

${ }^{13} \mathrm{C}$ NMR, $100 \mathrm{MHz}, \mathrm{CDCl}_{3}$ 